\title{
Hydrodynamic theory of flocking at a solid-liquid interface: long range order and giant number fluctuations
}

\author{
Niladri Sarkar, ${ }^{1,}$ Abhik Basu, ${ }^{2,}$ † and John Toner ${ }^{3}$, 用 \\ ${ }^{1}$ Instituut-Lorentz, Leiden University, P.O. Box 9506, 2300 RA Leiden, The Netherlands \\ ${ }^{2}$ Condensed Matter Physics Division, Saha Institute of Nuclear Physics, Calcutta 700064, West Bengal, India \\ ${ }^{3}$ Department of Physics and Institute of Theoretical Science, \\ University of Oregon, Eugene, Oregon 97403, USA
}

(Dated: June 28, 2021)

\begin{abstract}
We construct the hydrodynamic theory of coherent collective motion ("flocking") at a solidliquid interface. The polar order parameter and concentration of a collection of "active" (selfpropelled) particles at a planar interface between a passive, isotropic bulk fluid and a solid surface are dynamically coupled to the bulk fluid. We find that such systems are stable, and have longrange orientational order, over a wide range of parameters. When stable, these systems exhibit "giant number fluctuations", i.e., large fluctuations of the number of active particles in a fixed large area. Specifically, these number fluctuations grow as the $3 / 4$ th power of the mean number within the area. Stable systems also exhibit anomalously rapid diffusion of tagged particles suspended in the passive fluid along any directions in a plane parallel to the solid-liquid interface, whereas the diffusivity along the direction perpendicular to the plane is non-anomalous. In other parameter regimes, the system becomes unstable.
\end{abstract}

\section{INTRODUCTION}

Active fluids are fluids containing self-propelled units ("active particles"), each capable of converting stored or ambient free energy into motion. These intrinsically nonequilibrium systems often display orientationally ordered phases, both in living [1 5] and nonliving [6-10] systems. Such phases of active fluids exhibit many phenomena impossible in equilibrium orientationally ordered phases (e.g., nematics [11), among them spontaneous breaking of continuous symmetries in two dimensions [12 15], instability in the extreme Stokesian limit [16, and giant number fluctuations 17-19.

As in equilibrium systems, the "hydrodynamic" (i.e., long distance, long-time) behavior of active fluids depends crucially on whether or not momentum is conserved. In what has become the standard nomenclature of this field, systems lacking momentum conservation due to, e.g., friction with a substrate, are referred to as "dry" 14, 19, 22, while active fluids with momentum conservation are called "wet" 2326 .

In this paper, we treat a heretofore unconsidered class of systems that is a natural hybrid of these two cases: a collection of polar active particles at a solid-liquid interface. Our study is inspired by experiments on highdensity actin motility assays 27, in which highly concentrated actin filaments on a solid-fluid interface are propelled by motor proteins. Another experimental realization is the work of Bricard et al [28, 29], who studied the emergence of macroscopically directed motion in "Quincke rotators": a dilute collection of motile colloids, in contact with a solid substrate, and immersed

\footnotetext{
* niladri2002in@gmail.com

$\dagger$ abhik.123@gmail.com, abhik.basu@saha.ac.in

$\ddagger$ jjt@uoregon.edu
}

in a passive bulk electrolytic fluid. The colloids spontaneously roll, making them self propelled, when a sufficiently strong electric field is applied to the electrolytic fluid.

The hybrid system we consider here is in some ways reminiscent of the phenomenon of "wet to dry crossover" studied by other authors [30, 31. However, there are important differences. The systems studied by 30, 31] are fully two dimensional, but had both viscous and frictional damping. In such a problem, the asymptotic, longest wavelength behavior is that of dry active matter. In our case, as long as our bulk fluid is semi-infinite, the asymptotic long-distance behavior is completely different, even in its scaling, from dry active matter. The mixture of wet and dry behavior in our system is not a crossover phenomenon, only occurring over an intermediate range of length scales. Rather, it is the true, asymptotic, longwavelength (i.e., hydrodynamic) behavior (again, for a semi-infinite bulk passive fluid).

The systems that we consider here differ from both dry and wet active matter, as defined above, by having both friction from the underlying solid substrate and the long range hydrodynamic interactions due to the overlying bulk passive fluid. Very interestingly, the experiments of 27. found giant fluctuations of the active particle number in a fixed volume, with a standard deviation that scaled with $N^{0.8}$ in the ordered phase, where $N$ is the mean number in the volume. Although the exponent of 0.8 is the value predicted [17, 18, for dry polar active fluids, we will argue here that this is a coincidence, and that in fact polar fluids at a solid-liquid interface belong to an entirely different and heretofore unstudied universality class, with number fluctuations scaling like $N^{0.75}$, which probably lies within experimental error of the 0.8 exponent found in the experiments.

In this article, inspired by the experiments of Ref. 27, 28, we formulate the hydrodynamic theory for a collec- 
tion of polar, self propelled particles at the interface between a solid substrate and a bulk isotropic passive liquid that is semi-infinite in the $z$-direction, as illustrated in Fig. 1. We focus on the extreme Stokesian limit, in which the momentum of both the active particles and the bulk fluid is entirely negligible relative to viscous drag. The theory is then used to study the stability and fluctuations of a uniformly ordered active polar state of the particles.

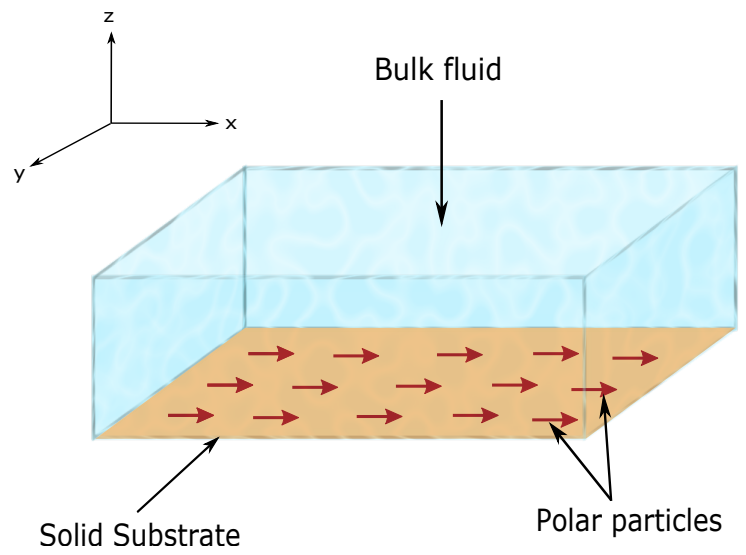

FIG. 1. (Color online) Schematic diagram of a layer of active polar particles embedded on a solid substrate surrounded by passive ambient fluid from the top

The governing equations of the active particle number density and polarization fluctuations in this system, which are introduced here for the first time are very different from those of "dry" and "wet" active matter". The breakdown of the Galilean invariance due to the friction from the substrate and the long ranged hydrodynamic interactions mediated by the passive bulk fluid lead to novel linear couplings between the density and polarization fluctuations, and non-local damping. The resultant behavior constitutes a new universality class, whose properties we describe in detail.

Our most surprising result is that this system can exhibit a long range ordered polar state, even in the presence of noise. This should be contrasted with both "wet" active systems, which are generically unstable [16] at very low Reynolds number, and with equilibrium systems, which cannot develop long ranged orientational order in two dimensions 32 35. Further, the number fluctuations in these systems are giant; i.e., the standard deviation $\sqrt{\left\langle(N-\langle N\rangle)^{2}\right\rangle}$ of the number $N$ of the active particles contained in a fixed open area grows with its average $\langle N\rangle$ much faster than its equilibrium off-critical counterpart. Specifically, we find

$$
\sqrt{\left\langle(N-\langle N\rangle)^{2}\right\rangle} \propto\langle N\rangle^{3 / 4} .
$$

We also find that the diffusivities of tagged particle in any direction parallel to the active fluid layer is logarithmically anomalous, by which we mean the mean squared displacement $\left\langle\left|\mathbf{r}_{\|}(t)\right|^{2}\right\rangle$ grows according to $<\left|r_{\|}(t)\right|^{2}>\propto$ $t \ln (t)$, in contrast to the usual linear in $t$ scaling of normal diffusion. Diffusion normal to the plane remains conventional, with $\left\langle\left|r_{\perp}(t)\right|^{2}\right\rangle \propto t$.

Unlike dry polar active matter, which can also exhibit long range order and giant number fluctuations [12 15, these systems do so even in a linear theory. Indeed, such a linear theory proves to provide an asymptotically exact long wavelength description of these systems, again in contrast to dry active matter.

The principal condition for stability of our systems is similar to the stability criterion for a simple compressible bulk fluid: the analog in our system of the bulk compressibility must be positive, as must our analog of the viscosities.

Thus, in contrast to "wet" active matter in the "Stokesian" limit [15, 16], our "mixed" system can be generically stable. Indeed, the requirements for stability are as easily met for these systems as for an equilibrium fluid.

These results are testable in standard experiments, e.g., in systems in which the active particles can be imaged, like those of [27, 28].

The remainder of this paper is organized as follows. We begin with a short summary of the technical results, which include the governing hydrodynamic equations themselves, in section II. In section (III), we formulate the linear hydrodynamic theory, including the equations of motion, for our system, and calculate the eigenfrequencies of small fluctuations about the perfectly ordered state. We thereby demonstrate that the ordered state is dynamically stable. In section $(\mathrm{V})$, we calculate the correlation functions implied by our equations of motion, and use them to show that long ranged polar order is robust against noise in these systems. In section (VI), we show that the density-density correlation functions found in section $(\mathrm{V})$ imply giant number fluctuations (equation (I.1)). We also show that those giant number fluctuations depend on the shape of the "counting volume", as well as the mean number of particles it contains, in contrast to equilibrium systems, in which the mean number alone determines the variance. In section (VIT), we calculate the velocity fields induced in the passive fluid by the active particles, and the correlations of those fields. We also consider the motion of tracer particles in the passive fluid, and thereby derive the anomalous diffusion (equations (II.5)-(II.11).) Finally, in section (IX), we use a dynamical renormalization group analysis to show that these linear results are asymptotically exact at long wavelengths. That is, we show that all non-linear terms allowed in the hydrodynamic equations of motion are irrelevant, in the renormalization group sense, in the long-wavelength limit. In appendix $\sqrt{\mathrm{A}}$, we solve the bulk Navier-Stokes equation to relate the passive fluid velocity throughout the bulk to the fluid velocity on the liquid-solid interface. In $(\mathrm{B})$, we derive the stability limit for the ordered state. In appendix (C), we evaluate an integral that arises in the calculation of the real-space correlation function. 


\section{SUMMARY OF THE PRINCIPAL RESULTS}

The variables of our hydrodynamic theory are two time-dependent fields that are confined to the plane of a flat interface between a solid substrate and a threedimensional (3D) passive, isotropic, bulk fluid. We choose our coordinates $(x, y, z)$ so that the interface sits at $z=0$, with the solid substrate underneath (i.e., $z<0$ ), and the bulk fluid above (i.e., $z>0$ ). We will denote two dimensional position within that plane by a two component vector $\mathbf{r}_{\|} \equiv(x, y)$. One of the hydrodynamic fields is the conserved density fluctuation $\delta \rho\left(\mathbf{r}_{\|}, t\right) \equiv \rho\left(\mathbf{r}_{\|}, t\right)-\rho_{0}$ of the areal density $\rho\left(\mathbf{r}_{\|}, t\right)$ about its mean value $\rho_{0}$.

Our second hydrodynamic variable reflects the polar order. By "polar", we mean each particle (labeled by an index $\alpha$, where $\alpha$ runs from 1 to the number of particles $N)$, can have associated with it a unique unit vector $\hat{\mathbf{p}}_{\alpha}$ (imagine, e.g., a collection of arrows, with $\hat{\mathbf{p}}_{\alpha}$ pointing

from the tail to the head of arrow $\alpha$ ).

To formulate hydrodynamics, we coarse grain this particle-based unit vector in to a position dependent vector field $\hat{\mathbf{p}}\left(\mathbf{r}_{\|}, t\right)$ defined on the surface at $z=0$.

Our theory studies small fluctuations around a uniform reference state with polarization $\mathbf{p}=\hat{\mathbf{x}}$ and density $\rho=$ $\rho_{0}$.

The linear hydrodynamic equations for the transverse orientation fluctuation $p_{y}$ and density fluctuation $\delta \rho$ for a polar-ordered flock at a solid-liquid interface will be systematically derived in section III below. They are obtained by by linearizing around a uniform reference state with polarization $\mathbf{p}=\hat{x}$ and density $\rho=\rho_{0}$. In Fourier space, these equations are

$$
\partial_{t} \delta \rho=-i v_{\rho}\left[q_{x} \delta \rho+\rho_{c} q_{y} p_{y}\right]+i \mathbf{q} \cdot \mathbf{f}_{\rho}
$$

Here, $v_{p}, v_{\rho}, \gamma, \gamma_{\rho}$, and $\rho_{c}$ are phenomenological parameters of our model, the first four of which have the dimensions of speed, while $\rho_{c}$ has the dimensions of (areal) density. The parameters $\gamma$ and $\gamma_{\rho}$ are damping coefficients that play roughly the same role in our problem as the viscosities play in a simple fluid 38 . The speeds $v_{\rho}$ and $v_{p}$ are fundamentally active parameters, with no analog in equilibrium fluids, which arise from the selfpropulsion of the active particles.

The Gaussian noises $\mathbf{f}_{\rho}$ and $f_{y}$ have zero mean and variances

$$
\begin{aligned}
& \left\langle f_{\rho i}\left(\mathbf{r}_{\perp}, t\right) f_{\rho j}\left(\mathbf{r}_{\perp}^{\prime}, t^{\prime}\right)\right\rangle=2 D_{\rho} \delta_{i j}^{s} \delta\left(\mathbf{r}_{\perp}-\mathbf{r}_{\perp}^{\prime}\right) \delta\left(t-t^{\prime}\right) \\
& \left\langle f_{y}\left(\mathbf{r}_{\perp}, t\right) f_{y}\left(\mathbf{r}_{\perp}^{\prime}, t^{\prime}\right)\right\rangle=2 D_{p} \delta\left(\mathbf{r}_{\perp}-\mathbf{r}_{\perp}^{\prime}\right) \delta\left(t-t^{\prime}\right),
\end{aligned}
$$

where $\mathbf{r}_{\perp}=(x, y)$ denotes the position of a point on the plane of the active fluid layer, and the parameters $D_{p, \rho}$ are constants with the dimensions of diffusion coefficients.

Fluctuations about the uniform ordered state are underdamped; that is, they both propagate and decay. The propagation is non-dispersive; that is, the wavespeed is independent of the wavenumber $q$, as in a simple compressible fluid. However, the wavespeed is direction dependent, as will be discussed in more detail below.

Even more strangely, the decay rate of these fluctuations scales completely differently from that of dry active matter; indeed, it scales linearly with $q$, just like the real part. This means that, unlike almost every other physical system that exhibits non-dispersive propagating modes [36, 37, the "quality factor" $Q$ of our system, defined as the ratio of the real part of $\omega(\mathbf{q})$ to its imaginary part, does not diverge as wavenumber $q$ goes to zero. Instead, it approaches a finite, system-dependent constant, independent of $q$, as $q \rightarrow 0$.

This unusual damping in turn leads to the aforementioned giant number fluctuations of the active particles, as given by equation (I.1).

We also find that, when the active particles at the interface are in their ordered state, they "stir" the passive fluid above them. The components $\left\langle v_{x}^{2}\left(\mathbf{r}_{\|}, z\right)\right\rangle$ and $\left\langle v_{y}^{2}\left(\mathbf{r}_{\|}, z\right)\right\rangle$ of the mean squared velocity thereby induced in the passive fluid are inversely proportional to the distance $z$ from the solid-fluid interface, whereas $\left\langle v_{z}^{2}\left(\mathbf{r}_{\|}, z\right)\right\rangle$ is inversely proportional to the cube of the distance $z$, i.e, it falls of as $1 / z^{3}$. Here, the $x, y$, and $z$ axes are respectively the direction of the polarization of the active particles, the in-plane direction orthogonal to that, and the normal to the interface, as illustrated in figure (1).

These predictions for the passive fluid velocity correlations could be tested experimentally by tracking neutrally buoyant passive tracer particles in the passive fluid. Such particle tracking thus provides a probe of the fluctuations of the active particles in the ordered state.

These velocity fluctuations in the passive fluid also exhibit long ranged spatio-temporal correlations, which decay as $1 / t$ for the projection of the velocity on the interface. The projection of the passive fluid velocity along $z$ (perpendicular to the surface) decay as $1 / t^{3}$.

These correlations in turn lead to anomalous diffusion 
of neutrally buoyant passive particles in the $x$ - and $y$ direction, with variances of the displacements obeying

$$
\begin{aligned}
& \left\langle(x(t)-x(0))^{2}\right\rangle=2 D_{x} t\left[\ln \left(\frac{v_{0} t}{z_{0}}\right)+O(1)\right], \quad t \ll \frac{z_{0}^{2}}{D_{z}}, \\
& \left\langle(y(t)-y(0))^{2}\right\rangle=2 D_{y} t\left[\ln \left(\frac{v_{0} t}{z_{0}}\right)+O(1)\right], \quad t \ll \frac{z_{0}^{2}}{D_{z}}, \\
& \left\langle(x(t)-x(0))(y(t)-y(0)\rangle=0, \quad t \ll \frac{z_{0}^{2}}{D_{z}} .\right.
\end{aligned}
$$

The cross-correlation function in the third line of (II.5) vanishes because the system is symmetric under inversion of $y$. Here, $v_{0}$ is a system-dependent characteristic speed associated with the active particles (roughly speaking, it is the speed at which they move over the surface due to their self-propulsion), $z_{0}$ is the initial distance of the neutrally buoyant particle from the surface, and $D_{x}, D_{y}$ are diffusion constants of the same order of magnitude as the diffusion constant in the $z$ direction.

The results (II.5) hold for times $t$ short compared to the time it takes for the neutrally buoyant particle to diffuse in the $z$ direction a distance comparable to $z_{0}$; that is, for $t \ll \frac{z_{0}^{2}}{D_{z}}$, where $D_{z}$ is the diffusion constant in the $z$-direction. This is a genuine diffusion constant; that is, the motion along $z$ is simple diffusion, with a $z$ independent diffusion constant. For $t \gg \frac{z_{0}^{2}}{D_{z}}$, after which the neutrally buoyant particle will have diffused in the $z$ direction a distance much larger than $z_{0}$, the behavior of $\left\langle(x(t)-x(0))^{2}\right\rangle$, and $\left\langle(y(t)-y(0))^{2}\right\rangle$ can be obtained by replacing $z_{0}$ in II.5 with $\sqrt{D_{z} t}$, which is just the rms distance the particle will have diffused away from the surface in that time. Doing so, we find that the motion on these much longer time scales is still superdiffusive, but with precisely half the "superdiffusion" constant. That is,

$$
\begin{aligned}
& \left\langle(x(t)-x(0))^{2}\right\rangle=D_{x} t\left[\ln \left(\frac{v_{0}^{2} t}{D_{z}}\right)+O(1)\right], \quad t \gg \frac{z_{0}^{2}}{D_{z}}, \\
& \left\langle(y(t)-y(0))^{2}\right\rangle=D_{y} t\left[\ln \left(\frac{v_{0}^{2} t}{D_{z}}\right)+O(1)\right], \quad t \gg \frac{z_{0}^{2}}{D_{z}},
\end{aligned}
$$

$\left\langle(x(t)-x(0))(y(t)-y(0)\rangle=0, \quad t \gg \frac{z_{0}^{2}}{D_{z}}\right.$,

Again, as in II.5 above, the cross-correlation in the third line of (II.8) vanishes because of symmetry under inversion of $y$. Diffusion in the $z$-direction remains conventional. Furthermore, the diffusion constant for diffusion in the $z$-direction is independent of height $z$. This set of predictions could also be tested experimentally by particle tracking of neutrally buoyant tracer particles in the passive fluid.

Particles denser than the passive fluid, which therefore sediment, will also be affected by this activity induced flow. We find that particles sedimenting from an initial height $z_{0}$ will, when they reach the surface, be spread over a region of dimensions $\sqrt{\left\langle\left(x(z=0)-x\left(z=z_{0}\right)\right)^{2}\right\rangle}$ and $\sqrt{\left\langle\left(y(z=0)-y\left(z=z_{0}\right)\right)^{2}\right\rangle}$ in the $x$ and $y$ directions, respectively, with

$$
\begin{aligned}
& \left\langle\left(x(z=0)-x\left(z=z_{0}\right)\right)^{2}\right\rangle=2 D_{x}\left(\frac{z_{0}}{v_{\text {sed }}}\right) \ln \left(\frac{v_{0}}{v_{\text {sed }}}\right), \quad v_{\text {sed }} \ll v_{0}, \\
& \left\langle\left(y(z=0)-y\left(z=z_{0}\right)\right)^{2}\right\rangle=2 D_{y}\left(\frac{z_{0}}{v_{\text {sed }}}\right) \ln \left(\frac{v_{0}}{v_{\text {sed }}}\right), \quad v_{\text {sed }} \ll v_{0}, \\
& \left\langle\left(x(z=0)-x\left(z=z_{0}\right)\right)\left(y(z=0)-y\left(z=z_{0}\right)\right\rangle=0 \quad, \quad v_{\text {sed }} \ll v_{0},\right.
\end{aligned}
$$

where $v_{0}$ is roughly the mean speed of the active particles, and $v_{\text {sed }}$ is the speed at which the sedimenting particles sink. These results only hold when the sedimenting speed $v_{\text {sed }} \ll v_{0}$; in the opposite limit, the lateral diffusion of a sedimenting particle becomes conventional.

Once again, these predictions should be readily testable in particle tracking experiments.

We turn now to correlations of the active particles themselves. These are determined by the characteristic frequencies of the fluctuations. We find that these eigenfrequencies $\omega(\mathbf{q})$ are complex, and given by

$$
\omega(\mathbf{q})=\Upsilon\left(\theta_{\mathbf{q}}\right) q
$$

Here $\Upsilon$ is a complex (and complicated!) function of the angle $\theta_{\mathbf{q}}$ between the wavevector $\mathbf{q}$ and the direction of spontaneous polarization of the active particles, and is the solution of a parameter and direction dependent quadratic equation given in section IV

Allowing the fluctuations of the stable modes to be driven by an additive white noise (which will arise from both noisy self-propulsion of the active particles, and thermal fluctuations), we find the orientational correlation function 


$$
C_{p p}(\mathbf{q}, \omega) \equiv\left\langle\left|p_{y}(\mathbf{q}, \omega)\right|^{2}\right\rangle=\left(\frac{1}{q^{2}}\right) F_{p p}\left(\left(\frac{\omega}{q}\right), \theta_{\mathbf{q}}\right),
$$

where $F_{p p}\left(\omega / q, \theta_{\mathbf{q}}\right)$ is a scaling function whose precise form is given in Section $V$. We also find that the peak positions of the scaling function, which we give in detail in Section V] agree with those found in the experiments of [29] on Quinke rotators.

The density-density correlation function and the orientation-density correlation function obey similar scaling laws:

$$
\begin{aligned}
& C_{\rho \rho}(\mathbf{q}, \omega) \equiv\left\langle\left|p_{y}(\mathbf{q}, \omega)\right|^{2}\right\rangle=\frac{1}{q^{2}} F_{\rho \rho}\left(\left(\frac{\omega}{q}\right), \theta_{\mathbf{q}}\right), \\
& C_{p \rho}(\mathbf{q}, \omega) \equiv\left\langle\rho(\mathbf{q}, \omega) p_{y}(-\mathbf{q},-\omega)\right\rangle=\frac{1}{q^{2}} F_{p \rho}\left(\left(\frac{\omega}{q}\right), \theta_{\mathbf{q}}\right),
\end{aligned}
$$

with slightly different scaling functions $F_{\rho \rho}\left(\omega / q, \theta_{\mathbf{q}}\right)$ and $F_{p \rho}\left(\omega / q, \theta_{\mathbf{q}}\right)$, whose precise forms are given by equations V.10 and (V.12) of section V.

Integrating these spatio-temporally Fouriertransformed correlation functions over all frequencies $\omega$ gives surprisingly simple expressions for the equal-time correlation functions. Specifically,

$$
C_{p p}(\mathbf{q}) \equiv\left\langle\left|p_{y}(\mathbf{q}, t)\right|^{2}\right\rangle=\int_{-\infty}^{\infty} \frac{d \omega}{2 \pi} C_{p p}(\mathbf{q}, \omega)=\frac{f_{p p}\left(\theta_{\mathbf{q}}\right)}{q},
$$

where the function $f_{p p}$ depends only on $\theta_{\mathbf{q}}$ and not $\mathbf{q}$, whose precise form is discussed later in Section $V$.

For the equal-time density autocorrelation function, we obtain

$$
C_{\rho \rho}(\mathbf{q}) \equiv\left\langle|\rho(\mathbf{q}, t)|^{2}\right\rangle=\frac{f_{\rho \rho}\left(\theta_{\mathbf{q}}\right)}{q}
$$

where $f_{\rho \rho}$ is another function of $\theta_{\mathbf{q}}$, to be given later.

Finally, the equal-time cross-correlation function is given by

$$
C_{p \rho}(\mathbf{q}) \equiv\left\langle p_{y}(\mathbf{q}, t) \rho(-\mathbf{q}, t)\right\rangle=\frac{f_{p \rho}\left(\theta_{\mathbf{q}}\right)}{q}
$$

where the direction dependence is given by the function $f_{p \rho}$ that depends only on $\theta_{\mathbf{q}}$.

Fourier transforming these in space leads to equally simple expressions for the real space, equal-time correlation functions, all of which scale as $1 / r$ with anisotropic amplitudes.

That is:

$$
\begin{aligned}
C_{p p}(\mathbf{r}) & \equiv\langle\delta \hat{\mathbf{p}}(\mathbf{r}+\mathbf{R}, t) \delta \hat{\mathbf{p}}(\mathbf{R}, t)\rangle \approx\left\langle p_{y}(\mathbf{r}+\mathbf{R}, t) p_{y}(\mathbf{R}, t)\right\rangle \\
& =\frac{f_{p p}\left(\theta_{\mathbf{r}}\right)}{r},
\end{aligned}
$$

where, the function $f_{p p}\left(\theta_{\mathbf{r}}\right)$ depends only on $\theta_{\mathbf{r}}$. the angle between $\mathbf{r}$ and the direction of the mean polarization, and not on the magnitude $r \equiv|\mathbf{r}|$. The precise form of $f_{p p}\left(\theta_{\mathbf{r}}\right)$ is given in equation blah of section blah.

For the real space equal-time density autocorrelation, we obtain

$$
C_{\rho \rho}(\mathbf{r}) \equiv\langle\delta \rho(\mathbf{r}+\mathbf{R}, t) \delta \rho(\mathbf{R}, t)\rangle=\frac{f_{\rho \rho}\left(\theta_{\mathbf{r}}\right)}{r}
$$

where $f_{\rho \rho}$ is another function of $\theta_{\mathbf{r}}$, to be given later.

Finally, the equal-time polarization-density crosscorrelation function is given by

$$
C_{p \rho}(\mathbf{r}) \equiv\left\langle p_{y}(\mathbf{r}+\mathbf{R}, t) \rho(\mathbf{R}, t)\right\rangle=\frac{f_{p \rho}\left(\theta_{\mathbf{q}}\right)}{q}
$$

where the direction dependence is given by the function $f_{p \rho}$ that depends only on $\theta_{\mathbf{r}}$.

These predictions could also be tested experimentally in systems in which the active particles can be imaged, like those of [27, 28].

\section{DERIVATION OF THE HYDRODYNAMIC THEORY}

\section{A. Identifying the hydrodynamic variables}

We consider active polar particles of areal density $\rho\left(\mathbf{r}_{\|}, t\right)$ confined to a flat interface between a solid substrate and a three-dimensional (3D) passive, isotropic, bulk fluid. We choose our coordinates $(x, y, z)$ so that the interface sits at $z=0$, with the solid substrate underneath (i.e., $z<0$ ), and the bulk fluid above (i.e., $z>0$ ). By "polar", we mean each particle (labeled by an index $\alpha$, where $\alpha$ runs from 1 to the number of particles $N$ ), can have associated with it a unique unit vector $\hat{\mathbf{p}}_{\alpha}$. (Imagine, e.g., a collection of arrows, with $\hat{\mathbf{p}}_{\alpha}$ pointing from the tail to the head of arrow $\alpha$.)

To formulate hydrodynamics, we coarse grain this particle-based unit vector in to a position dependent vector field $\hat{\mathbf{p}}\left(\mathbf{r}_{\|}, t\right)$ defined on the surface at $z=0$. In principle, this coarse graining can lead to fluctuations in the magnitude of the coarse grained $\hat{\mathbf{p}}\left(\mathbf{r}_{\|}, t\right)$ as well as its direction. However, since we are considering a system in an orientationally ordered state, not at a continuous transition from an ordered to a disordered state, fluctuations of the magnitude $|\hat{\mathbf{p}}|$ of $\hat{\mathbf{p}}$ relax fast; i.e., the relaxation time-scale remains finite in the long wavelength limit. This is because fluctuations in the magnitude of $|\hat{\mathbf{p}}|$, unlike fluctuations in its direction, are not Goldstone modes associated with the spontaneous breaking of continuous rotation invariance. Therefore, there is no symmetry reason for them to be slow, so we expect them to be fast generically. Hence, in the spirit of hydrodynamics, we ignore these magnitude fluctuations, and set $\hat{\mathbf{p}}^{2}=1$ without any loss of generality. A renormalization group analysis 40] confirms this heuristic argument.

We choose our coordinate system such that $p_{x}=1$ is the reference polar ordered state (see figure (1). 
In the presence of friction from the substrate, there is no momentum conservation on the surface, so the only conserved variable on the surface of relevance for the present problem is the active particle number. The hydrodynamic theory that we develop below thus includes the active particle number density $\rho\left(\mathbf{r}_{\|}, t\right)$ and the orientational order parameter $\hat{\mathbf{p}}\left(\mathbf{r}_{\|}, t\right)$ fluctuations as the relevant slow or "hydrodynamic" variables. We also include the bulk fluid velocity $\mathbf{v}(\mathbf{r}, t)$, which is defined throughout the semi-infinite three dimensional space above the surface, since in that space momentum (which is equivalent to velocity in the limit of an incompressible bulk fluid) is conserved. However, we will work in the Stokesian limit, in which viscous forces dominate inertial ones; this has the effect of "enslaving" the bulk velocity to the hydrodynamic variables $\rho\left(\mathbf{r}_{\|}, t\right)$ and $\hat{\mathbf{p}}\left(\mathbf{r}_{\|}, t\right)$ on the surface, as we will show below.

\section{B. Hydrodynamic equations of motion}

We will formulate the hydrodynamic equations for these variables by expanding their equations of motion phenomenologically in powers of fluctuations of both fields $\hat{\mathbf{p}}$ and $\rho$ from their mean values, and in spatiotemporal gradients. In so doing, we must respect all symmetries and conservation laws of the underlying dynamics. Because this is a non-equilibrium system, however, additional constraints that would apply in equilibrium, such as detailed balance, do not hold here, and any term consistent with the symmetries and conservation laws is allowed, and therefore will, in general, be present.

We will consider systems with underlying rotational invariance in the plane of the surface. That is, we will focus on the case in which there is no a priori preferred direction in that plane; the active particles are free to spontaneously break this symmetry, by choosing a direction in which to spontaneously point.

With these considerations in mind, we will now derive the hydrodynamic equations of motion for the fields $\rho\left(\mathbf{r}_{\|}, t\right), \hat{\mathbf{p}}\left(\mathbf{r}_{\|}, t\right)$, and the bulk velocity velocity $\mathbf{v}\left(\mathbf{r}_{\|}, z, t\right)$.

We begin with the active particle density $\rho\left(\mathbf{r}_{\|}, t\right)$.

\section{Equation of motion for the active particle density $\rho$}

The conservation of the active particles implies that they obey a continuity equation:

$$
\partial_{t} \rho+\nabla_{s} \cdot \mathbf{J}_{\rho}=0
$$

where $\boldsymbol{\nabla}_{s} \equiv \hat{\mathbf{x}} \partial / \partial x+\hat{\mathbf{y}} \partial / \partial y$ is the $2 \mathrm{D}$ gradient operator, with $\hat{\mathbf{x}}$ and $\hat{\mathbf{y}}$ the unit vectors along the $x$ and $y$ axis respectively. The active particle current $\mathbf{J}_{\rho}$ is generated by friction with the bulk fluid velocity $\mathbf{v}\left(\mathbf{r}=\mathbf{r}_{\|}, z=0\right)$, plus additional diffusive and active currents. We will phenomenologically expand all of these effects to leading order in powers of the bulk velocity evaluated at the surface $\mathbf{v}\left(\mathbf{r}_{\|}, z=0\right)$, and gradients, keeping all terms consistent with the underlying rotation invariance. In practice, this means we can make the vector $\mathbf{J}_{\rho}$ only out of vectors the active particle configuration itself chooses, that is, out of gradients, the surface velocity $\mathbf{v}_{s}\left(\mathbf{r}_{\|}, t\right)$, by which we mean the bulk velocity evaluated at the surface; i.e., $\mathbf{v}_{s}\left(\mathbf{r}_{\|}, t\right) \equiv \mathbf{v}\left(\mathbf{r}_{\|}, z=0, t\right)$, and the polarization $\hat{\mathbf{p}}\left(\mathbf{r}_{\|}, t\right)$. These constraints force $\mathbf{J}_{\rho}$ to take the form:

$$
\mathbf{J}_{\rho}\left(\mathbf{r}_{\|}\right)=\rho_{e}\left(\rho,\left|\mathbf{v}_{s}\right|\right) \mathbf{v}_{s}(x, y, z=0)+\kappa\left(\rho,\left|\mathbf{v}_{s}\right|\right) \hat{\mathbf{p}}-D_{\rho} \boldsymbol{\nabla}_{s} \rho-D_{\rho 2} \hat{\mathbf{p}}\left(\hat{\mathbf{p}} \cdot \nabla_{s}\right) \rho-\boldsymbol{f}_{\rho}(x, y) .
$$

to leading order in gradients, where $D_{\rho}>0$ is the diffusion constant, and $D_{\rho 2}$ is an anisotropic diffusion coefficient. Any other symmetry-permitted vector terms involving additional gradients, e.g., $\nabla_{s}^{2} \hat{\mathbf{p}}$ would be irrelevant in the long wavelength limit. Note also that the first two terms on the rhs of (III.2) reflect the fact that the the "active particle velocity" $\mathbf{J}_{\rho} / \rho$ will, in general, be different from the velocity $\mathbf{v}_{s}$ of the bulk fluid at the surface, due to the activity. The existence of a non-zero $D_{\rho 2}$ reflects the fact that, once the underlying rotation invariance is broken by the development of a spontaneous polarization $\hat{\mathbf{p}}$, diffusion along the direction of $\hat{\mathbf{p}}$ need not, and, in general, will not, proceed at the same speed as diffusion perpendicular to that direction. The "effective density" $\rho_{e}\left(\rho,\left|\mathbf{v}_{s}\right|\right)$ would simply be $\rho$ itself in a Galilean invariant model, but since the solid surface breaks Galilean invariance, it can in general depend non-linearly on $\rho$, and even on the magnitude $\left|\mathbf{v}_{s}\right|$ of the surface velocity. All of these dependences disappear in the linear model; furthermore, our analysis in section IX of the non-linear model shows that these dependences do not affect any of our predictions for the long distance behavior of the system. More precisely, they can all be absorbed into a suitable, finite "renormalization" of the parameters of the linear theory. In renormalization group jargon, they are "irrelevant". We have only included them in the current IIII.2 to make our starting model more general.

The factor $\kappa\left(\rho,\left|\mathbf{v}_{s}\right|\right)$ is an active parameter reflecting the self-propulsion of the particles through interaction with the solid substrate. It can also in principle depend on $\rho$ and the magnitude $\left|\mathbf{v}_{s}\right|$ of the surface velocity. As 
for $\rho_{e}$, all of these dependences disappear in the linear model, and prove to also be irrelevant in the RG sense just described.

For completeness, we have included in this current a zero-mean Gaussian white noise $\boldsymbol{f}_{\rho}$ that we take to be delta-correlated in space and time:

$$
\left\langle f_{\rho i}(x, y, t) f_{\rho j}\left(x^{\prime}, y^{\prime}, t^{\prime}\right)\right\rangle=2 D_{\rho} \delta_{i j}^{s} \delta\left(x-x^{\prime}\right) \delta\left(y-y^{\prime}\right) \delta\left(t-t^{\prime}\right)
$$

This noise is added to model the stochastic nature of the density dynamics. In the equilibrium limit, this would have been a thermal noise representing the effects of a finite temperature and with a variance proportional to the diffusivity, as required by the Fluctuation-Dissipation theorem [41. In an active system such as ours, there is no constraint like the fluctuation-dissipation theorem or the Einstein relations relating the noise variance (III.3) to the diffusivity. The white noise (III.3) in (III.2) reappears in (III.1) as a conserved noise, consistent with the density being a conserved variable; see Eq. (III.4) below. However, as we will show later, this noise proves to be irrelevant in the long wavelength limit compared to the polarization noise we will introduce later. The diffusion constants $D_{\rho}$ and $D_{\rho 2}$ prove to be irrelevant in the longwavelength limit as well, as we shall see.

Using (III.2) in (III.1), the continuity equation (III.1) can be written

$$
\begin{aligned}
\partial_{t} \rho= & -\hat{\mathbf{p}} \cdot \nabla_{s} \kappa(\rho)-\kappa(\rho) \nabla_{s} \cdot \hat{\mathbf{p}} \\
& +\nabla_{s} \cdot\left[D_{\rho} \nabla_{s} \rho+D_{\rho 2} \hat{\mathbf{p}}\left(\hat{\mathbf{p}} \cdot \nabla_{s}\right) \rho\right] \\
& -\rho_{e}\left(\rho,\left|\mathbf{v}_{s}\right|\right) \nabla_{s} \cdot \mathbf{v}_{s}-\mathbf{v}_{s} \cdot \nabla_{s} \rho_{e}\left(\rho,\left|\mathbf{v}_{s}\right|\right)+\nabla_{s} \cdot \mathbf{f}_{\rho} .
\end{aligned}
$$

\section{The bulk velocity $\mathbf{v}\left(\mathbf{r}_{\|}, z, t\right)$}

In calculating the bulk velocity $\mathbf{v}\left(\mathbf{r}_{\|}, z, t\right)$, we will assume the bulk fluid is in the extreme "Stokesian" limit, in which inertia is negligible relative to viscous drag. This should be appropriate for most systems in which the active particles are microscopic, since the Reynolds' number will be extremely low for such particles.

The 3D incompressible bulk velocity field $\mathbf{v}=$ $\left(v_{i}, v_{z}\right), i=x, y$ satisfies the $3 \mathrm{D}$ Stokes' equation

$$
\eta \nabla_{3}^{2} v_{\alpha}\left(\mathbf{r}_{\|}, z\right)=\partial_{\alpha} \Pi\left(\mathbf{r}_{\|}, z\right)
$$

where the incompressibility constraint

$$
\nabla_{3} \cdot \mathbf{v}=0
$$

determines $\Pi$, the hydrostatic pressure. In these expressions (III.5), (III.6), $\boldsymbol{\nabla}_{3} \equiv \hat{\mathbf{x}} \partial / \partial x+\hat{\mathbf{y}} \partial / \partial y+\hat{\mathbf{z}} \partial / \partial z$ is the full three-dimensional gradient operator, with $\hat{\mathbf{x}}, \hat{\mathbf{y}}$, and $\hat{\mathbf{z}}$ the unit vectors along the $x, y$, and $z$ axes respectively.
We could, in principle, add a random Brownian noise to this Stokesian force balance equation (III.5) to reflect thermal fluctuations in the non-zero temperature bulk fluid. However, since the bulk fluid is passive - that is, equilibrium, we know that the net effect of such a noise term on the surface polarization must be to introduce a purely equilibrium random noise in the polarization equation of motion. So we will instead take the simpler approach of simply adding such a thermal noise by hand to the active noises that will also appear in that equation.

In the absence of such a noise term, the noiseless Stokes equation III.5) can be solved exactly for the bulk velocity $\mathbf{v}\left(\mathbf{r}_{\|}, z, t\right)$ in terms of the surface velocity $\mathbf{v}_{s}\left(\mathbf{r}_{\|}, t\right)$. If we Fourier expand the surface velocity:

$$
\mathbf{v}_{s}\left(\mathbf{r}_{\|}, t\right)=\frac{1}{\sqrt{L_{x} L_{y}}} \sum_{\mathbf{q}} \mathbf{v}_{s}(\mathbf{q}, t) e^{i \mathbf{q} \cdot \mathbf{r}_{\|}}
$$

where $\left(L_{x}, L_{y}\right)$ are the linear dimensions of our (presumed rectangular) surface, then, as we show in Appendix $\mathrm{A}$, the bulk velocity $\mathbf{v}\left(\mathbf{r}_{\|}, z, t\right)$ is given by

$\mathbf{v}\left(\mathbf{r}_{\|}, z, t\right)=\frac{1}{\sqrt{L_{x} L_{y}}} \sum_{\mathbf{q}}\left[\mathbf{v}_{s}-z\left(\mathbf{q} \cdot \mathbf{v}_{s}\right)(\hat{\mathbf{q}}+i \hat{\mathbf{z}})\right] e^{-q z+i \mathbf{q} \cdot \mathbf{r}_{\perp}}$

This is to be supplemented by the boundary conditions at $z=\infty$, at which all stresses vanish, and at $z=0$, which we will now discuss. For a bulk passive fluid with a free surface, the boundary condition is given by the vanishing of the appropriate component of the shear stress. On the other hand, for a bulk fluid resting on a solid surface, one imposes the "no-slip" boundary condition that the relative velocity between the solid surface and the fluid layer in contact vanishes. More generally, if there is a slip velocity between the solid surface and the liquid layer in contact with the solid surface, then one must impose what in fluid dynamics is known as a partial slip condition on the fluid layer in contact with the solid substrate. For an active fluid layer at the solid-liquid interface, the fluid layer in contact is active; hence, there should be active forces acting on this layer. Generalizing the partial-slip boundary condition [42] for an active surface fluid, we impose the following active boundary condition at $z=0$ :

$$
\begin{aligned}
& \mathbf{v}\left(\mathbf{r}_{\|}, z=0, t\right) \equiv \mathbf{v}_{s}\left(\mathbf{r}_{\|}, t\right) \\
& =v_{a}(\rho) \hat{\mathbf{p}}\left(\mathbf{r}_{\|}, z=0, t\right)+\mu \boldsymbol{\tau}_{\|}\left(\mathbf{r}_{\|}, z=0, t\right),
\end{aligned}
$$

where $v_{a}(\rho)$ is the spontaneous self-propulsion speed of the active particles relative to the solid substrate that can depend upon the local active particle density, and the force density $\boldsymbol{\tau}_{\|}$parallel to the surface is given by

$\boldsymbol{\tau}_{\|}\left(\mathbf{r}_{\|}, z=0, t\right)=\mathcal{O} \hat{\mathbf{z}} \cdot \boldsymbol{\sigma}^{\text {bulk }}\left(\mathbf{r}_{\|}, z=0, t\right)+\boldsymbol{\tau}^{\text {surface }}\left(\mathbf{r}_{\|}, t\right)$

with $\mathcal{O}$ the operator that projects any vector onto the plane of the surface, and $\sigma^{\text {bulk }}$ the bulk stress tensor. 
The projection operator $\mathcal{O}$ is given by

$$
\mathcal{O}_{i j}=\delta_{i j}-\delta_{i z} \delta_{j z}
$$

while the bulk stress tensor is given by

$$
\sigma_{i j}^{\mathrm{bulk}}=\eta\left(\partial_{i} v_{j}+\partial_{j} v_{i}\right)-\Pi \delta_{i j}
$$

The $\eta$ term in III.12 is simply the viscous stress in the bulk fluid, while the pressure term $\Pi \delta_{i j}$ drops out of the force density $\boldsymbol{\tau}_{\|}$parallel to the surface due to the projection operator $\mathcal{O}$.

The form of this boundary condition is dictated by symmetry. Essentially, any vector in the two dimensional plane of the solid surface that is spontaneously chosen by the system (as opposed to specified a priori, is allowed, and, therefore, will be present. Because the projection $\mathcal{O} \hat{\mathbf{z}} \cdot \boldsymbol{\sigma}^{\text {bulk }}$ is such a vector, a term proportional to it is allowed, and, hence, will generically be present in the boundary condition (III.9).
Physically, we expect contributions to the surface stress from the bulk fluid stress since this stress is also experienced at the interface where the active particles are situated. So we would expect this stress in general to influence the active particles, and, therefore, the boundary condition on the bulk fluid velocity at the interface on which these particles sit. Note that if we set both the active surface stress $\tau^{\text {surface }}$ and the self-propulsion speed $v_{a}$ to zero, equations II.8 to II.11 reduce to the conventional partial slip boundary condition of passive fluid dynamics 42 .

Requiring that the bulk stress tensor (III.12) be divergenceless (as it must be in the Stokesian limit, since, by definition, in that limit the viscous stresses dominate the inertial ones) leads to the Stokes' equation (III.5) for the bulk flow. Note that $\tau^{\text {surface }}$ exists only at the interface at $z=0$ and hence does not appear in the Stokes' equation for the bulk flow. The surface force $\boldsymbol{\tau}^{\text {surface }}\left(\mathbf{r}_{\|}, t\right)$, when expanded to leading order in the polarization $\hat{\mathbf{p}}$ and gradients, must, by symmetry, take the form [43, 44]

$$
\tau_{i}^{\text {surface }}=\zeta_{1}(\rho) \hat{\mathbf{p}} \cdot \nabla_{S} p_{i}+\zeta_{2}(\rho) p_{i} \nabla_{S} \cdot \hat{\mathbf{p}}+p_{i} \hat{\mathbf{p}} \cdot \nabla_{S} \zeta(\rho)-\partial_{i} P_{s}^{\prime}(\rho)
$$

In (III.13), the $\zeta_{1,2}$ and $\zeta$ represent active stresses, and $P_{s}^{\prime}$ is a surface osmotic pressure (not to be confused with the bulk pressure $\Pi$ in the Stokes equation (III.5)), both of which depend on the density of the active particles.
Inserting III.12 and III.13 into our expression III.10 for the force density $\tau_{\|}$parallel to the surface, and using the result in our active boundary condition (III.9, gives, for $i=(x, y)$,

$$
v_{s i}\left(\mathbf{r}_{\|}, t\right)=v_{a}(\rho) p_{i}\left(\mathbf{r}_{\|}, t\right)+\zeta_{1}(\rho) \hat{\mathbf{p}} \cdot \nabla_{S} p_{i}+\zeta_{2}(\rho) p_{i} \nabla_{S} \cdot \hat{\mathbf{p}}+p_{i} \hat{\mathbf{p}} \cdot \nabla_{S} \zeta(\rho)+\mu \eta\left(\frac{\partial v_{i}\left(\mathbf{r}_{\|}, z, t\right)}{\partial z}\right)_{z=0}-\partial_{i} P_{s}
$$

where we have defined $P_{s} \equiv \mu P_{s}^{\prime}$, and used the fact that the velocity normal to the surface vanishes at the surface (i.e., $v_{z}(z=0)=0$ ) 43 . Note that the velocity $v_{i}\left(\mathbf{r}_{\|}, z, t\right)$ is $i$ 'th component the full bulk velocity.

The term proportional to $\partial_{z} v_{i}$ can be given another interpretation: if we define a length $a \equiv \mu \eta, \mu \eta\left(\frac{\partial v_{i}}{\partial z}\right)_{z=0}=$ $a\left(\frac{\partial v_{i}}{\partial z}\right)_{z=0}$ is simply the velocity of a fluid element a distance $a$ above the surface. Hence, we can alternatively interpret this term as modeling active particles of finite thickness $a$ on the surface which are passively convected by the local fluid velocity at that height. For a system in thermal equilibrium, $v_{a}=0=\zeta_{1,2}(\rho)=\zeta(\rho)$, and III.14 reduces to the well-known equilibrium partial slip boundary condition.

\section{Equation of motion for the polarization $\hat{\mathbf{p}}$}

We now turn to the equation of motion for $\hat{\mathbf{p}}$. As the active particles are polar, the system is not invariant under $\hat{\mathbf{p}} \rightarrow-\hat{\mathbf{p}}$ symmetry. This means that terms even in $\hat{\mathbf{p}}$ are allowed in our phenomenological expression for $\partial_{t} \hat{\mathbf{p}}$.

The most general equation of motion for $p_{k}$ allowed by symmetry is therefore:

$$
\partial_{t} p_{k}=T_{k i}\left(\alpha v_{s i}-\lambda_{p v}\left(\mathbf{v}_{s} \cdot \nabla_{s}\right) p_{i}+\frac{h_{i}}{\gamma_{1}}+\left(\frac{\nu_{1}-1}{2}\right) p_{j} \partial_{i} v_{s j}+\left(\frac{\nu_{1}+1}{2}\right)\left(\hat{\mathbf{p}} \cdot \nabla_{s}\right) v_{s i}-\lambda\left(\hat{\mathbf{p}} \cdot \nabla_{s}\right) p_{i}-\partial_{i} P_{p}(\rho)+f_{i}\right)
$$

where we have defined the transverse projection operator

$$
T_{k i} \equiv \delta_{k i}^{s}-p_{k} p_{i}
$$

which projects any vector orthogonal to $\hat{\mathbf{p}}$. Its presence 
in III.15) insures that the fixed length condition $|\hat{\mathbf{p}}|=1$ on $\hat{\mathbf{p}}$ is preserved.

In (III.15), the "molecular field" $\mathbf{h}$ is given by

$$
\mathbf{h}=-\frac{\delta F}{\delta \hat{\mathbf{p}}},
$$

where the Frank free energy is

$$
F=\frac{1}{2} \int d^{2} r_{\perp}\left[K_{1}\left(\nabla_{S} \cdot \hat{\mathbf{p}}\right)^{2}+K_{3}\left|\nabla_{S} \times \hat{\mathbf{p}}\right|^{2}\right] .
$$

Here, $K_{1}$ and $K_{3}$ are respectively the Frank splay and bend elastic modulii. This $h_{i} / \gamma_{1}$-term, together with the Frank free energy $F$, gives the damping terms in III.50) below. Notice that the more familiar classical alignmentfree energy term $K\left|\nabla_{S} \hat{\mathbf{p}}\right|^{2}$ is already implicitly included in (III.18) separately, as can be seen by using the vector calculus identity

$$
\left|\nabla_{S} \times \hat{\mathbf{p}}\right|^{2}=\left|\nabla_{S} \hat{\mathbf{p}}\right|^{2}-\left(\nabla_{S} \cdot \hat{\mathbf{p}}\right)^{2}-\nabla_{S} \cdot \mathbf{W},
$$

where $\mathbf{W} \equiv\left(\hat{\mathbf{p}} \cdot \nabla_{S}\right) \hat{\mathbf{p}}-\hat{\mathbf{p}}\left(\nabla_{S} \cdot \hat{\mathbf{p}}\right)$, in (III.18). Doing so, and dropping the surface term arising from the total divergence in (III.19), we can rewrite (III.18) in the form

$$
F=\frac{1}{2} \int d^{2} r_{\perp}\left[\left(K_{1}-K_{3}\right)\left(\nabla_{S} \cdot \hat{\mathbf{p}}\right)^{2}+K_{3}\left|\nabla_{S} \hat{\mathbf{p}}\right|^{2}\right] .
$$

In this form, it is explicit that we have included the usual alignment-free energy term proportional to $\left|\nabla_{S} \hat{\mathbf{p}}\right|^{2}$ in (III.18).

The reasoning that leads to (III.15) is essentially identical to that leading to our earlier expression (III.14 for the active boundary condition: we want to include $e v$ ery vector spontaneously picked by the system, provided that vector is also leading order in an expansion in powers of spatial gradients. It is straightforward to see that (III.15) does so. It contains all of the vectors contained in (III.14), except for an explicit term proportional to $\hat{\mathbf{p}}$ itself. We need not include such a term in (III.15), since it would be "killed" by he projection operator $T_{k i}$. All the other terms in (III.15) are vectors spontaneously picked by the system. Furthermore, they all have independent coefficients, since there is no symetry argument that would lock them ito any particular relation.

Note that terms proportional to some a priori specified vector, say $\hat{\mathbf{x}}$, are not allowed in (III.15), since they violate rotation invariance by singling out a special direction that is not spontaneously chosen by the system (i.e., by any of the vectors $\hat{\mathbf{p}}, \mathbf{v}$, etc., or by spatial gradients).

The fact that we are performing a gradient expansion allows us to drop terms proportional to, e.g., $\nabla_{S}^{2} v_{s i}$, $\nabla_{S}^{4} v_{s i}$, and so on, in (III.15). While such terms are certainly allowed by symmetry, they are irrelevant at long distances relative to the $\alpha v_{s i}$ and $p_{j} \partial_{i} v_{s j}$ terms already present in (III.15).

When all such symmetry forbidden and irrelevant in the gradient expansion terms are dropped, one is left with III.15) as the only possible equation of motion for $\hat{\mathbf{p}}$.
To summarize, the equation of motion (III.15) includes all of the relevant nonequilibrium terms allowed by symmetry, in addition to the usual equilibrium terms and the convective covariant derivative of $\hat{\mathbf{p}}$. Here, the presence of the solid substrate underneath breaks any Galilean invariance, and hence a term directly proportional to $v_{i}$ in III.15 is permissible: indeed the $\alpha$-term in III.15) clearly breaks the Galilean invariance. The presence of this terms implies flow alignment or antialignment of the polarization $\hat{\mathbf{p}}$ depending upon the sign of $\alpha$ in Eq. (III.15). The "polarization pressure" $P_{p}(\rho)$ is an additional function of the density, independent of the "osmotic pressure" $P_{s}(\rho)$ introduced earlier. Furthermore, the $\lambda$ term represents active self advection. Finally, the terms proportional to $\nu_{1}$ are "flow alignment terms", identical in form to those found in nematic liquid crystals [4].

We have also added to the equation of motion (III.15) a white noise $\mathbf{f}$ with statistics

$$
\left\langle f_{i}\left(\mathbf{r}_{\perp}, t\right) f_{j}\left(\mathbf{r}_{\perp}^{\prime}, t^{\prime}\right)\right\rangle=2 D_{p} \delta_{i j} \delta\left(\mathbf{r}_{\perp}-\mathbf{r}_{\perp}^{\prime}\right) \delta\left(t-t^{\prime}\right) .
$$

As discussed earlier, this noise also incorporates equilibrium contributions from thermal fluctuations of the bulk fluid. These must, by the fluctuation-dissipation theorem, be spatiotemporally white, as we have assumed in (III.21), since the equilibrium dynamics of $\hat{\mathbf{p}}$ is local in space and time. The actual noise strength $D_{p}$ in (III.21) is larger than the equilibrium value (which is proportional to $k_{B} T$ ) due to active contributions to the noise.

\section{Summary of the equations of motion}

Our hydrodynamic model, then, is summarized by the equations of motion (III.4) and III.15) for $\rho$ and $\hat{\mathbf{p}}$, respectively, and the solution (III.8) of the Stokes equation (III.5) for the bulk velocity field $\mathbf{v}(x, y, z, t)$ obtained with the boundary condition (III.14). When considering fluctuations, we will also need the noise correlations (III.3) and (III.21) .

\section{Linearization of the equations of motion}

We now show that linearizing Eqs. (III.4) and (III.15) about a uniform reference state with polarization $\mathbf{p}=\hat{x}$ and density $\rho=\rho_{0}$ produces Eqs. (II.1) and (II.2) in section II.

The equations of motion and boundary conditions found in the previous section have an obvious spatially uniform, steady state solution:

$$
\begin{array}{r}
\rho\left(\mathbf{r}_{\|}, t\right)=\rho_{0} \\
\hat{\mathbf{p}}\left(\mathbf{r}_{\|}, t\right)=\hat{\mathbf{x}} \\
\mathbf{v}_{s}\left(\mathbf{r}_{\|}, t\right)=v_{0} \hat{\mathbf{x}} \\
\mathbf{v}\left(\mathbf{r}_{\|}, z, t\right)=v_{0} \hat{\mathbf{x}}
\end{array}
$$


where we have defined

$$
v_{0} \equiv v_{a}\left(\rho_{0}\right)
$$

and have chosen the $\hat{\mathbf{x}}$ axis of our coordinate system to be along the (spontaneously chosen) direction of polarization, as illustrated in figure (1).

As a first step towards understanding fluctuations about this steady state, we will write

$$
\begin{aligned}
& \rho\left(\mathbf{r}_{\|}, t\right)=\rho_{0}+\delta \rho\left(\mathbf{r}_{\|}, t\right), \\
& \hat{\mathbf{p}}\left(\mathbf{r}_{\|}, t\right)=\hat{\mathbf{x}} \sqrt{1-p_{y}^{2}\left(\mathbf{r}_{\|}, t\right)}+p_{y}\left(\mathbf{r}_{\|}, t\right) \hat{\mathbf{y}}, \\
& \mathbf{v}_{s}\left(\mathbf{r}_{\|}, t\right)=\left(v_{0}+\delta v_{s x}\left(\mathbf{r}_{\|}, t\right)\right) \hat{\mathbf{x}}+v_{s y}\left(\mathbf{r}_{\|}, t\right) \hat{\mathbf{y}},
\end{aligned}
$$

and expand the equations of motion III.4) and (III.15) for $\rho$ and $\hat{\mathbf{p}}$, and the boundary condition (III.14), to linear order in $\delta \rho$ and $p_{y}$. We will obtain the bulk velocity $\mathbf{v}\left(\mathbf{r}_{\|}, z, t\right)$ from the surface velocity $\mathbf{v}_{s}\left(\mathbf{r}_{\|}, t\right)$ using our solution (III.8) of the Stokes equation.

We will expand the surface osmotic pressure of the particles $P_{s}(\rho)$ to linear order in $\delta \rho$ :

$$
P_{s}(\rho)=\sigma \delta \rho .
$$

We will also expand the "surface polarization pressure" $P_{p}(\rho)$ to linear order in $\delta \rho$ (and will show later that higher powers of $\delta \rho$ are irrelevant at long wavelengths):

$$
P_{p}(\rho)=\sigma_{p} \delta \rho,
$$

and likewise expand the $\zeta^{\prime}$ 's in equation III.14 . To linear order, it is sufficient to take

$$
\begin{aligned}
& \zeta_{1}(\rho)=\zeta_{1}\left(\rho_{0}\right) \equiv \zeta_{10} \\
& \zeta_{2}(\rho)=\zeta_{2}\left(\rho_{0}\right) \equiv \zeta_{20} \\
& \zeta(\rho)=\zeta\left(\rho_{0}\right)+\bar{\zeta} \delta \rho
\end{aligned}
$$

Finally, we expand $v_{a}(\rho), \kappa(\rho)$ and $\rho_{e}(\rho)$ to linear order in $\delta \rho$ :

$$
\begin{aligned}
& v_{a}(\rho) \equiv v_{0}+v_{a}^{\prime} \delta \rho, \\
& \kappa(\rho) \equiv \kappa_{0}+\bar{\kappa} \delta \rho, \\
& \rho_{e}(\rho)=\rho_{1}+\rho_{e}^{\prime} \delta \rho,
\end{aligned}
$$

where $\kappa_{0}, \bar{\kappa}, \rho_{1}$, and $\rho_{e}^{\prime}$ are all constants to linear order. All of the other parameters (i.e., the diffusion constants $D_{\rho}$ and $D_{\rho 2}, v_{a}, \alpha, \lambda, \lambda_{p v}, \gamma_{1}$, and $\nu_{1}$ can to linear order be replaced by their values at $\rho=\rho_{0},\left|\mathbf{v}_{s}\right|=v_{0}$, and treated as constants.

Now using our active boundary condition Eq. (III.14, the fluctuating velocity components $\delta v_{x}$ and $v_{y}$ can be expressed up to linear order as

$$
\begin{aligned}
\delta v_{s x} & =v_{a}^{\prime} \delta \rho+\bar{\zeta} \partial_{x} \delta \rho+\mu \eta\left(\partial_{z} v_{x}\right)_{z=0}+\zeta_{20} \partial_{y} p_{y}-\sigma \partial_{x} \delta \rho, \\
v_{s y} & \left.=v_{0} p_{y}+\mu \eta\left(\partial_{z} v_{y}\right)_{z=0}+\zeta_{10} \partial_{x} p_{y}-\sigma \partial_{y} \delta \rho .38\right)
\end{aligned}
$$

These expressions for $\delta v_{s x}$ and $v_{s y}$ are implicit equations, since the bulk velocities $v_{x}$ and $v_{y}$ on the right hand side also depend on the surface velocity through III.8) .

We can make them explicit by solving them iteratively. This is most conveniently done in Fourier space, because we can then obtain the iterative solution to lowest order in powers of the wavenumber $q$.

Performing a two-dimensional Fourier transform on (III.38) and (III.39) - that is, Fourier transforming over $\mathbf{r}_{\|}$, and using our solution (III.8) for the bulk velocity in terms of the surface velocity, we obtain

$$
\begin{aligned}
& \delta v_{s x}(\mathbf{q}, t)=v_{a}^{\prime} \delta \rho(\mathbf{q}, t)+i \bar{\zeta} q_{x} \delta \rho(\mathbf{q}, t)+\mu \eta W_{x}(\mathbf{q}, t)+i \zeta_{20} q_{y} p_{y}(\mathbf{q}, t)-i \sigma q_{x} \delta \rho(\mathbf{q}, t) \\
& \delta v_{s y}(\mathbf{q}, t)=v_{0} p_{y}(\mathbf{q}, t)+\mu \eta W_{y}(\mathbf{q}, t)+i \zeta_{10} q_{x} p_{y}(\mathbf{q}, t)-i \sigma q_{y} \delta \rho(\mathbf{q}, t)
\end{aligned}
$$

where we have defined

$$
\begin{aligned}
& \mathbf{W}(\mathbf{q}, t) \equiv\left(\partial_{z}\left\{\left[\mathbf{v}_{s}(\mathbf{q}, t)-z\left(\mathbf{q} \cdot \mathbf{v}_{s}(\mathbf{q}, t)\right)(\hat{\mathbf{q}}+i \hat{\mathbf{z}})\right] e^{-q z}\right\}\right)_{z=0} \\
& =-q \mathbf{v}_{s}(\mathbf{q}, t)-\left(\mathbf{q} \cdot \mathbf{v}_{s}(\mathbf{q}, t)\right) \hat{\mathbf{q}}+W_{z}(\mathbf{q}, t) \hat{\mathbf{z}}
\end{aligned}
$$

the leading order in $q$ terms (which are $O(q)$ ) come from the $v_{a}^{\prime} \delta \rho$ term in (III.40) and the $v_{0} p_{y}$ term in (III.41). Keeping only those terms implies

$$
\mathbf{v}_{s}(\mathbf{q}, t) \approx v_{a}^{\prime} \delta \rho \hat{\mathbf{x}}+v_{0} p_{y}(\mathbf{q}, t) \hat{\mathbf{y}}
$$


Inserting this into III.42 gives

$$
\begin{aligned}
& W_{x}=-\left(\frac{q^{2}+q_{x}^{2}}{q}\right) v_{a}^{\prime} \delta \rho-v_{0}\left(\frac{q_{x} q_{y}}{q}\right) p_{y}, \\
& W_{y}=-v_{a}^{\prime}\left(\frac{q_{x} q_{y}}{q}\right) \delta \rho-v_{0}\left(\frac{q^{2}+q_{y}^{2}}{q}\right) p_{y} .
\end{aligned}
$$

Inserting these expressions back into (III.40 and (III.41) gives us our final closed form expression for the two components of the surface velocity, written entirely in terms of $p_{y}$ and $\delta \rho$ :

$$
\begin{aligned}
\delta v_{s x}(\mathbf{q}, t) & =\left[v_{a}^{\prime}+i(\bar{\zeta}-\sigma) q_{x}-\mu \eta v_{a}^{\prime}\left(\frac{q^{2}+q_{x}^{2}}{q}\right)\right] \delta \rho(\mathbf{q}, t)+\left[i \zeta_{20} q_{y}-\mu \eta v_{0}\left(\frac{q_{x} q_{y}}{q}\right)\right] p_{y}(\mathbf{q}, t) \\
v_{s y}(\mathbf{q}, t) & =\left[v_{0}-\mu \eta\left(\frac{q^{2}+q_{y}^{2}}{q}\right)+i \zeta_{10} q_{x}\right] p_{y}(\mathbf{q}, t)-\left[i \sigma q_{y}+\mu \eta v_{a}^{\prime}\left(\frac{q_{x} q_{y}}{q}\right)\right] \delta \rho
\end{aligned}
$$

Note the non-analytic character of the $\mu \eta$ terms in these expressions; this reflects the long-ranged hydrodynamic interaction between active particles on the surface mediated by the bulk passive fluid. Indeed, it is only through these terms that the presence of the bulk fluid makes itself felt. Note also that these terms are real; we'll see in a moment that this makes them damping terms. They are also the same order in $q$ as the imaginary $\zeta$ and $\sigma$ terms, which are associated with propagation. This is the origin of the peculiar property of our system that damping and propagation are the same order in wavevector (or, equivalently, that the quality factor $Q$ of the normal modes of this system is finite and independent of wavenumber $q$ at small $q$ ). This should be contrasted with, e.g., a simple bulk equilibrium fluid, for which the propagating terms are $O(q)$, while the damping terms are $O\left(q^{2}\right)$.

Note that the parameter $\zeta_{20}$ has dropped out at this point in our calculation, because it only leads to terms of higher order in $q$ than we have kept here.

With these expressions (III.46) and (III.47) for the surface velocity in terms of $p_{y}$ and $\delta \rho$ in hand, we can now derive closed equations of motion for $p_{y}$ and $\delta \rho$. First, we must linearize the general equations of motion (III.4) and (III.15), using the linearizations (III.31) - (III.37) for the pressures and $\zeta$ 's, and (III.27) and (III.28) for the density and the polarization. Doing so for the continuity equation (III.4), we obtain

$$
\partial_{t} \delta \rho=-\kappa_{0} \partial_{y} p_{y}-\left(\bar{\kappa}+v_{0} \rho_{e}^{\prime}\right) \partial_{x} \delta \rho-\rho_{1} \nabla_{s} \cdot \mathbf{v}_{s}+\left[D_{\rho} \nabla_{s}^{2} \rho+D_{\rho 2} \partial_{x}^{2}\right] \delta \rho+\nabla_{s} \cdot \mathbf{f}_{\rho}
$$

Fourier transforming equation (III.48), and keeping only

terms to leading order in $q$, we find

$$
\partial_{t} \delta \rho(\mathbf{q}, t)=-i \kappa_{0} q_{y} p_{y}-i\left(\bar{\kappa}+v_{0} \rho_{e}^{\prime}\right) q_{x} \delta \rho-i \rho_{1} \mathbf{q} \cdot \mathbf{v}_{s}+i \mathbf{q} \cdot \mathbf{f}_{\rho}
$$

Setting $k=y$ in the equation of motion III.15 for $\hat{\mathbf{p}}$, and linearizing the resulting equation gives

$\partial_{t} p_{y}=\alpha\left(v_{s y}-v_{0} p_{y}\right)-\left(\lambda_{p v} v_{0}+\lambda\right) \partial_{x} p_{y}+\left(\frac{\nu_{1}-1}{2}\right) \partial_{y} \delta v_{s x}+\left(\frac{\nu_{1}+1}{2}\right) \partial_{x} v_{s y}-\sigma_{p} \partial_{y} \delta \rho+\frac{1}{\gamma_{1}}\left(K_{1} \partial_{y}^{2}+K_{3} \partial_{x}^{2}\right) p_{y}+f_{y}$ 
particular combination appears is not an accident, but, rather, a consequence of rotation invariance: if we work to zeroeth order in gradients in our solution for the surface velocity, this term vanishes, as it must, since $p_{y}$ is a Goldstone mode associated with the spontaneous breaking of the continuous rotational symmetry. It is because of this exact cancellation that we needed to evaluate $\mathbf{v}_{s}$ to higher order in gradients of $p_{y}$ in (III.40) and (III.41, which is why we had to keep higher order gradient terms in that expression. Note that no such cancellation happened when we calculated the vector $\mathbf{W}$ eqn. (III.42) earlier, so there we could truncate the expansion for the surface velocity at zeroeth order in the gradient term.

Fourier transforming equation (III.50), and keeping only terms to leading order in $q$, we find that the molecular field terms proportional to the Frank constants $K_{1,3}$ are higher order in $q$, and so can be dropped, leaving us with

$$
\left.\partial_{t} p_{y}=\alpha\left(v_{s y}-v_{0} p_{y}\right)-i\left(\lambda_{p v} v_{0}+\lambda\right) q_{x} p_{y}+i\left(\frac{\nu_{1}-1}{2}\right) q_{y} \delta v_{s x}+i\left(\frac{\nu_{1}+1}{2}\right) q_{x} v_{s y}-i \sigma_{p} q_{y} \delta \rho+f_{y}\right)
$$

Now inserting our expressions (III.46) and (III.47) for the surface velocity in terms of $p_{y}$ and $\delta \rho$ into the Fourier transformed equations of motion (III.49 and (III.51, and keeping only terms to leading order in $q$, gives our final closed form for the linearized equations of motion:

$$
\partial_{t} \delta \rho=-i v_{\rho}\left[q_{x} \delta \rho+\rho_{c} q_{y} p_{y}\right]+i \mathbf{q} \cdot \mathbf{f}_{\rho}
$$

$$
\partial_{t} p_{y}=-i v_{p} q_{x} p_{y}-\gamma\left(\frac{q^{2}+q_{y}^{2}}{q}\right) p_{y}-\left(\frac{\gamma_{\rho}}{\rho_{c}}\right)\left(\frac{q_{x} q_{y}}{q}\right) \delta \rho-i \sigma_{t} q_{y} \delta \rho+f_{y}
$$

where we have defined the characteristic velocities

$$
v_{p} \equiv \lambda_{p v} v_{0}+\lambda-\zeta_{10} \alpha-\left(\frac{\nu_{1}+1}{2}\right) v_{0}
$$

and

$$
v_{\rho} \equiv \bar{\kappa}+\rho_{e}^{\prime} v_{0}+\rho_{1} v_{a}^{\prime}
$$

the total inverse compressibility $\sigma_{t} \equiv \sigma_{p}+\sigma \alpha-$ $\left(\frac{\nu_{1}-1}{2}\right) v_{a}^{\prime}$, the characteristic density

$$
\rho_{c} \equiv \frac{\rho_{1} v_{0}+\kappa_{0}}{v_{\rho}}
$$

and the bulk fluid damping coefficients $\gamma \equiv \alpha v_{0} \mu \eta$ and $\gamma_{\rho} \equiv \alpha \mu \eta v_{a}^{\prime} \rho_{c}$. Both $\gamma$ and $\gamma_{\rho}$ have the dimensions of speed. Further more, all the contributions from $\delta v_{x}(\mathbf{q}, t)$ in (III.46) in (III.51) are subleading and hence do not appear in (III.52), where only the terms leading order in $\mathbf{q}$ are kept.

We thus obtain the linearized hydrodynamic equations (III.51) and (III.52), which are nothing but equations (II.1) and (II.2) mentioned earlier. These are invariant under $q_{y} \rightarrow-q_{y}, p_{y} \rightarrow-p_{y}, \rho \rightarrow \rho$, but not invariant under $q_{x} \rightarrow-q_{x}$. This is equivalent to invariance in real space under $y \rightarrow-y, p_{y} \rightarrow-p_{y}, \rho \rightarrow \rho$, but with no analogous invariance under $x \rightarrow-x$. In these two equations $v_{\rho} \neq v_{p}$ in general, due to the abscence of Galilean invariance because of friction with the solid substrate. The effective coefficient $\gamma$, when positive (which can be achieved by tuning the signs of the various original model parameters), serves as the effective damping coefficient in the model. Interestingly, the effective damping here is $\mathcal{O}(q)$, which is far stronger than the $\mathcal{O}\left(q^{2}\right)$ damping in the linearized Toner-Tu model for flocking [13, 14] in the hydrodynamic limit. Hydrodynamic interactions mediated by the passive, bulk fluid above are responsible for this $\mathcal{O}(q)$ damping.

An alert reader might wonder how hydrodynamic interactions mediated by the bulk fluid can dominate friction from the underlying solid substrate, which, after all, is $O\left(q^{0}\right)$. The reason is that friction with the substrate, while playing a very important role (in particular, it is the principal mechanism limiting the speed $v_{0}$ of the active particles), does not act to suppress fluctuations in the directions of motion of those particles (or, similarly, the polarization. The leading order damping of such fluctuations, which are the Goldstone modes of our problem, come from the hydrodynamic interactions. 


\section{MODE STRUCTURE OF THE LINEARIZED EQUATIONS AND STABILITY OF THE UNIFORM STATE}

Having written down the equations of motion, we will now analyze the linear stability of the system. We work in polar coordinates $\mathbf{q}=\left(q \cos \theta_{\mathbf{q}}, q \sin \theta_{\mathbf{q}}\right)$, and set the noises to zero. Assuming a time-dependence of the form $\rho, p_{y} \sim \exp (-i \omega t)$, this leads to the eigenvalue condition on $\omega$ :

$$
\omega^{2}+i \omega\left[i\left(v_{p}+v_{\rho}\right) q \cos \theta_{\mathbf{q}}+\gamma q\left(1+\sin ^{2} \theta_{\mathbf{q}}\right)\right]-i\left[i v_{p} q \cos \theta_{\mathbf{q}}+\gamma q\left(1+\varphi \sin ^{2} \theta_{\mathbf{q}}\right)\right] v_{\rho} q \cos \theta_{\mathbf{q}}-c_{0}^{2} q^{2} \sin ^{2} \theta_{\mathbf{q}}=0,
$$

where we have defined

$$
c_{0}^{2} \equiv \sigma_{t} \rho_{c} v_{\rho}
$$

and

$$
\varphi \equiv 1-\frac{\gamma_{\rho}}{\gamma}
$$

The eigenfrequencies always scale as $q$, independent of all other parameters, as can be seen by inserting the ansatz

$$
\omega=\Upsilon\left(\theta_{\mathbf{q}}\right) q
$$

into (IV.1). This leads to a $q$ independent condition on $\Upsilon$ :

$$
\Upsilon^{2}+i \Upsilon\left[i\left(v_{p}+v_{\rho}\right) \cos \theta_{\mathbf{q}}+\gamma\left(1+\sin ^{2} \theta_{\mathbf{q}}\right)\right]-i\left[i v_{p} \cos \theta_{\mathbf{q}}+\gamma\left(1+\varphi \sin ^{2} \theta_{\mathbf{q}}\right)\right] v_{\rho} \cos \theta_{\mathbf{q}}-c_{0}^{2} \sin ^{2} \theta_{\mathbf{q}}=0
$$

thereby proving that $\omega$ scales like $q$.

For stability, we must have $\operatorname{Im}(\omega)<0$ for all $\mathbf{q}$, which means $\operatorname{Im}\left(\Upsilon\left(\theta_{\mathbf{q}}\right)\right)<0$ for all $\theta_{\mathbf{q}}$. We show in appendix (B) that this condition is satisfied for sufficiently small $\gamma_{\rho}$ (which appears in (IV.5 through $\varphi \equiv 1-\frac{\gamma_{\rho}}{\gamma}$ ), provided $\gamma$ and $c_{0}^{2}$ are both $>0$. Note that this condition is similar to the stability criterion for a simple compressible bulk fluid: $c_{0}^{2}>0$ simply means the bulk compressibility is positive, while $\gamma>0$ is analogous to requiring positive shear and bulk viscosities. The stability condition on $\gamma_{\rho}$ is

$$
-|\varpi-1|-\sqrt{(\varpi-1)^{2}+\frac{1}{m^{2}}}<\frac{\gamma_{\rho}}{\gamma}<-|\varpi-1|+\sqrt{(\varpi-1)^{2}+\frac{1}{m^{2}}},
$$

where we have defined the "Mach number"

$$
m \equiv \frac{v_{\rho}}{c_{0}},
$$

and the speed ratio

$$
\varpi \equiv \frac{v_{p}}{v_{\rho}} .
$$

It is easy to see that this condition can always be satisfied for sufficiently small $\gamma_{\rho}$; in particular, the allowed region always includes $\gamma_{\rho}=0$. That a sufficiently large $\gamma_{\rho}$ relative to the effective damping $\gamma$ can lead to instabilities is not surprising. A non-zero $\gamma_{\rho}$ (or, equivalently, $v_{a}^{\prime}$ ) implies that different patches of the system move at different speeds. Of course, damping tends to homogenize the density by exchanging particles, thereby reducing the speed differences. However, if $\gamma_{\rho}$ is too large, damping may not be sufficient to suppress these speed fluctuations. The eventual steady state may be nonuniform, leading to a patterned state, though its actual nature cannot be ascertained from the linearized equations of motion. Nonlinear amplitude equations would be necessary for a full-fledged analysis of the steady state. It will be interesting to explore the relation between these instabilities here and the banding instabilities reported in [46, 47.

Thus generic underdamped propagating waves with anisotropic, $\theta_{\mathbf{q}}$-dependent wavespeed proportional to $q$ are expected for the wide range of parameters satisfying the stability condition derived in appendix B. 


\section{CORRELATION FUNCTIONS AND ROBUSTNESS OF LONG-RANGED ORDER AGAINST NOISE}

In the stable region of the parameter space, the correlation functions for the system in the steady state may be calculated from the noise-driven equations of motion. Dropping for now the density force $\mathbf{f}_{\rho}$ (we will show later that it is irrelevant in the long-wavelength limit), and solving the linear equations of motion for the spatio-temporally Fourier transformed fields $p_{y}(\mathbf{q}, \omega)$ and $\delta \rho(\mathbf{q}, \omega)$ gives

$$
p_{y}(\mathbf{q}, \omega)=\frac{i\left(\omega-v_{\rho} q \cos \theta_{\mathbf{q}}\right) f_{y}(\mathbf{q}, \omega)}{\left(\omega-c_{+}\left(\theta_{\mathbf{q}}\right) q\right)\left(\omega-c_{-}\left(\theta_{\mathbf{q}}\right) q\right)+i\left(\omega \Psi\left(1, \theta_{\mathbf{q}}\right)-v_{\rho} q \Psi\left(\varphi, \theta_{\mathbf{q}}\right) \cos \theta_{\mathbf{q}}\right) q}
$$

$$
\delta \rho(\mathbf{q}, \omega)=\frac{i \rho_{c} v_{\rho} q \sin \theta_{\mathbf{q}} f_{y}(\mathbf{q}, \omega)}{\left(\omega-c_{+}\left(\theta_{\mathbf{q}}\right) q\right)\left(\omega-c_{-}\left(\theta_{\mathbf{q}}\right) q\right)+i\left(\omega \Psi\left(1, \theta_{\mathbf{q}}\right)-v_{\rho} q \Psi\left(\varphi, \theta_{\mathbf{q}}\right) \cos \theta_{\mathbf{q}}\right) q}
$$

where we have defined

$$
\Psi(\varphi, \theta) \equiv \gamma\left(1+\varphi \sin ^{2} \theta\right)
$$

sitions of the peaks in the scaling function versus $u$ [39, precisely those found for dry active matter in [13 15]; i.e.,

and with the "sound speeds" $c_{ \pm}\left(\theta_{\mathbf{q}}\right)$, defined by the po-

$$
c_{ \pm}\left(\theta_{\mathbf{q}}\right)=\left(\frac{v_{\rho}+v_{p}}{2}\right) \cos \theta_{\mathbf{q}} \pm \sqrt{\frac{1}{4}\left(v_{\rho}-v_{p}\right)^{2} \cos ^{2} \theta_{\mathbf{q}}+c_{0}^{2} \sin ^{2} \theta_{\mathbf{q}}}
$$

The "sound speeds" $c_{ \pm}\left(\theta_{\mathbf{q}}\right)$ in (V.4), defined by the positions of the peaks in the scaling function versus $u$ [39], are precisely those found for dry active matter in 1315. Further, these peak positions agree with those found in the experiments of [29] on Quinke rotators. In Fig. (2) we show a polar plot of these sound speeds.
Autocorrelating these fields with themselves then gives their spatio-temporally Fourier transformed correlations:

$$
C_{p p}(\mathbf{q}, \omega) \equiv\left\langle\left|p_{y}(\mathbf{q}, \omega)\right|^{2}\right\rangle=\frac{2 D_{p}\left(\omega-v_{\rho} q \cos \theta_{\mathbf{q}}\right)^{2}}{\left(\omega-c_{+}\left(\theta_{\mathbf{q}}\right) q\right)^{2}\left(\omega-c_{-}\left(\theta_{\mathbf{q}}\right) q\right)^{2}+\left(\omega \Psi\left(1, \theta_{\mathbf{q}}\right)-v_{\rho} q \Psi\left(\varphi, \theta_{\mathbf{q}}\right) \cos \theta_{\mathbf{q}}\right)^{2} q^{2}}
$$

$$
C_{\rho \rho}(\mathbf{q}, \omega) \equiv\left\langle|\delta \rho(\mathbf{q}, \omega)|^{2}\right\rangle=\frac{2 D_{p} \rho_{c}^{2} v_{\rho}^{2} q^{2} \sin ^{2} \theta_{\mathbf{q}}}{\left(\omega-c_{+}\left(\theta_{\mathbf{q}}\right) q\right)^{2}\left(\omega-c_{-}\left(\theta_{\mathbf{q}}\right) q\right)^{2}+\left(\omega \Psi\left(1, \theta_{\mathbf{q}}\right)-v_{\rho} q \Psi\left(\varphi, \theta_{\mathbf{q}}\right) \cos \theta_{\mathbf{q}}\right)^{2} q^{2}}
$$


where we have used (III.21) to obtain the autocorrelation $\left\langle\left|f_{y}(\mathbf{q}, \omega)\right|^{2}\right\rangle=2 D_{p}$.

Similar reasoning gives the cross-correlation function

$$
C_{p \rho}(\mathbf{q}, \omega) \equiv\left\langle\rho(\mathbf{q}, \omega) p_{y}(-\mathbf{q},-\omega)\right\rangle=\frac{2 D_{p} \rho_{c} v_{\rho} q \sin \theta_{\mathbf{q}}\left(\omega-v_{\rho} q \cos \theta_{\mathbf{q}}\right)}{\left(\omega-c_{+}\left(\theta_{\mathbf{q}}\right) q\right)^{2}\left(\omega-c_{-}\left(\theta_{\mathbf{q}}\right) q\right)^{2}+\left(\omega \Psi\left(1, \theta_{\mathbf{q}}\right)-v_{\rho} q \Psi\left(\varphi, \theta_{\mathbf{q}}\right) \cos \theta_{\mathbf{q}}\right)^{2} q^{2}}
$$

Pulling a factor of $q^{2}$ out of the numerator of each of these expressions, and a factor of $q^{4}$ out of their denominators, gives the scaling forms (II.13), II.14, and
II.15, with the scaling functions $F_{p p}\left(u_{f}\right), F_{\rho \rho}\left(u_{f}\right)$ and $F_{p \rho}\left(u_{f}\right)$.

The scaling function $F_{p p}\left(u_{f}, \theta_{\mathbf{q}}\right)$ is given by

$$
F_{p p}\left(u_{f}, \theta_{\mathbf{q}}\right)=\frac{2 D_{p}\left(u_{f}-v_{\rho} \cos \theta_{\mathbf{q}}\right)^{2}}{\left(u_{f}-c_{+}\left(\theta_{\mathbf{q}}\right)\right)^{2}\left(u_{f}-c_{-}\left(\theta_{\mathbf{q}}\right)\right)^{2}+\left(u_{f} \Psi\left(1, \theta_{\mathbf{q}}\right)-v_{\rho} \Psi\left(\varphi, \theta_{\mathbf{q}}\right) \cos \theta_{\mathbf{q}}\right)^{2}},
$$

where $u_{f} \equiv \omega / q$.

Note that the existence of the scaling form (II.13) implies that the ratio of the widths of the peaks in $C_{p p}(\mathbf{q}, \omega)$, plotted versus $\omega$ for fixed $\mathbf{q}$, to their positions does not change as $\mathbf{q} \rightarrow \mathbf{0}$; this is what we meant by our earlier cryptic comment that the "quality factor $Q$ " becomes independent of $q$.

In Fig. (2), we show a polar plot of the sound speeds $c_{ \pm}\left(\theta_{\mathbf{q}}\right)$, which are the positions of the peaks in the scaling function $F_{p p}$ when plotted versus the scaling argument $u_{f}$ for fixed direction of propagation $\theta_{\mathbf{q}}$. As such, they are the analog in our system of the sound speeds in a simple compressible fluid.

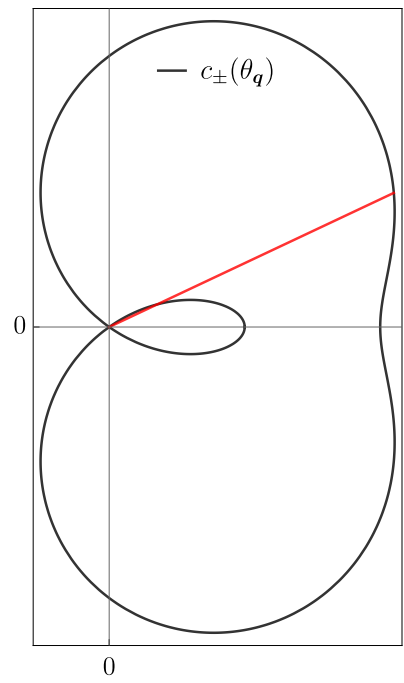

FIG. 2. (Color online) Polar plot of the sound speeds (V.4) (black curve). Here we have taken $q=1, v_{\rho}=1, v_{p}=$ $c_{0}=2$, and $\gamma=.3$ (all in arbitrary units). The polarization points directly to the right. The red straight line indicates the direction of $\mathbf{q}$ for which the scaling function $F_{p p}$ is plotted in figure (3).
The density-density correlation function obeys a similar scaling law:

$$
C_{\rho \rho}(\mathbf{q}, \omega) \equiv\left\langle\left|p_{y}(\mathbf{q}, \omega)\right|^{2}\right\rangle=\frac{1}{q^{2}} F_{\rho \rho}\left(\left(\frac{\omega}{q}\right), \theta_{\mathbf{q}}\right),
$$

with a slightly different scaling function $F_{\rho \rho}\left(u_{f}, \theta_{\mathbf{q}}\right)$ given by 


$$
F_{\rho \rho}\left(u_{f}, \theta_{\mathbf{q}}\right)=\frac{2 D_{p} \rho_{c}^{2} v_{\rho}^{2} \sin ^{2} \theta_{\mathbf{q}}}{\left(u_{f}-c_{+}\left(\theta_{\mathbf{q}}\right)\right)^{2}\left(u_{f}-c_{-}\left(\theta_{\mathbf{q}}\right)\right)^{2}+\left(u_{f} \Psi\left(1, \theta_{\mathbf{q}}\right)-v_{\rho} \Psi\left(\varphi, \theta_{\mathbf{q}}\right) \cos \theta_{\mathbf{q}}\right)^{2}}
$$

The orientation-density correlation function also obeys

a similar scaling law:

$$
C_{p \rho}(\mathbf{q}, \omega) \equiv\left\langle\rho(\mathbf{q}, \omega) p_{y}(-\mathbf{q},-\omega)\right\rangle=\frac{1}{q^{2}} F_{p \rho}\left(\left(\frac{\omega}{q}\right), \theta_{\mathbf{q}}\right),
$$

where the scaling function for this correlation function is

$$
F_{p \rho}\left(u_{f}, \theta_{\mathbf{q}}\right)=\frac{2 D_{p} \rho_{c} v_{\rho} \sin \theta_{\mathbf{q}}\left(u_{f}-v_{\rho} \cos \theta_{\mathbf{q}}\right)}{\left(u_{f}-c_{+}\left(\theta_{\mathbf{q}}\right)\right)^{2}\left(u_{f}-c_{-}\left(\theta_{\mathbf{q}}\right)\right)^{2}+\left(u_{f} \Psi\left(1, \theta_{\mathbf{q}}\right)-v_{\rho} \Psi\left(\varphi, \theta_{\mathbf{q}}\right) \cos \theta_{\mathbf{q}}\right)^{2}}
$$

These three scaling functions are plotted versus the scaling argument $u$ for a fixed direction of propagation $\theta_{\mathbf{q}}$ in Figure (3). Note that the two peaks in the polarization scaling function $F_{p p}$ are exactly the same height, but have different widths. We have chosen to plot this figure for a fairly generic direction of propagation. In contrast, for $\theta=\pi / 2$ (that is, propagation perpendicular to the polarization, which corresponds to a vertical line in figure (2)), the scaling functions $F_{p p}$ and $F_{\rho \rho}$ both become even functions of the scaling argument $u$, with two symmetrically placed peaks at $\pm c_{0}$. This implies that the correlation functions both become even functions of $\omega$, with two symmetrically placed peaks at $\pm c_{0} q$. Furthermore, for this direction of propagation, the two correlations functions are precisely proportional to each other; that is, their ratio is a constant, independent of both $q$ and $\omega$.

Another special direction is $\theta_{\mathbf{q}}=0$; i.e., propagation along the polarization. In this case, the asymmetry is maximized: the polarization scaling function has only one peak, at scaling argument $u=v_{p}$, meaning the correlation function has a single peak at $\omega=v_{p} q$. The density-density scaling function on the other hand, vanishes identically in this limit (except at the singular point $\omega=v_{\rho} q$, where its value depends on precisely how the limit $\omega \rightarrow v_{\rho} q, \theta_{\mathbf{q}}$ is approached).

Both of these special directions are, indeed, special: for generic directions of propagation $\theta \neq 0, \pm \pi / 2$, the scaling functions, and, hence, the $\omega$-dependence of the correlation functions, look like figure (3). That is, each scaling function has two in general asymmetrically placed peaks, in the same positions for both correlation functions.

The way the special limit $\theta=0$ is approached as $\theta \rightarrow 0$ is that the first (i.e., leftmost) peak of the polarization scaling function $F_{p p}$, while its height stays the same as that of the second (i.e., rightmost) peak, has its width continuously vanish as $\theta \rightarrow 0$, while the density scaling function $F_{\rho \rho}$ has the height of its first peak continuously vanish as $\theta \rightarrow 0$. One can see the tendency towards this limit in the plot of figure (3) at $\theta=27^{\circ}$.

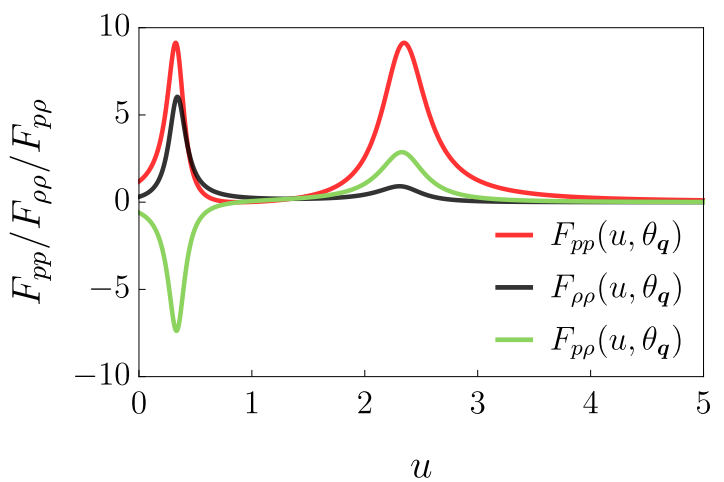

FIG. 3. (Color online) Plot of the scaling functions $F_{p p}\left(u_{f}, \theta_{\mathbf{q}}\right)$ ( red curve), $F_{\rho \rho}\left(\left(u_{f}, \theta_{\mathbf{q}}\right)\right.$ ( black curve), and $F_{p \rho}\left(u_{f}, \theta_{\mathbf{q}}\right)$ (green curve) versus scaling argument $u_{f} \equiv \frac{\omega}{q}$ for fixed $\theta_{\mathbf{q}}$. Here we have taken $v_{\rho}=1, v_{p}=c_{0}=2, \gamma=.3$, $D_{p}=.5, \rho_{c}=1, \gamma_{\rho} / \gamma=1 / 2$ (all in arbitrary units), and $\theta=27^{\circ}$ (the direction indicated by the straight line in figure (2).

We can use these expressions to calculate the equal time correlation functions; we find for the polarization $p_{y}$

$C_{p p}(\mathbf{q}) \equiv\left\langle\left|p_{y}(\mathbf{q}, t)\right|^{2}\right\rangle=\int_{-\infty}^{\infty} \frac{d \omega}{2 \pi}\left\langle\left|p_{y}(\mathbf{q}, \omega)\right|^{2}\right\rangle=\frac{D_{p}}{\pi} \int_{-\infty}^{\infty} \frac{\left(\omega-v_{\rho} q \cos \theta_{\mathbf{q}}\right)^{2} d \omega}{\left(\omega-c_{+}\left(\theta_{\mathbf{q}}\right) q\right)^{2}\left(\omega-c_{-}\left(\theta_{\mathbf{q}}\right) q\right)^{2}+\left(\omega \Psi\left(1, \theta_{\mathbf{q}}\right)-v_{\rho} q \Psi\left(\varphi, \theta_{\mathbf{q}}\right) \cos \theta_{\mathbf{q}}\right)^{2} q^{2}}$

Introducing a new variable of integration

$$
s \equiv \frac{\omega-v_{\rho} q \cos \theta_{\mathbf{q}} \Xi\left(\varphi, \theta_{\mathbf{q}}\right)}{\Psi\left(1, \theta_{\mathbf{q}}\right) q}
$$


where we have defined

$$
\Xi(\Phi, \theta) \equiv \frac{\Psi(\Phi, \theta)}{\Psi(1, \theta)}=\frac{1+\Phi \sin ^{2} \theta}{1+\sin ^{2} \theta}
$$

gives

$$
C_{p p}(\mathbf{q})=\frac{D_{p}}{\pi \Psi\left(1, \theta_{\mathbf{q}}\right) q} \int_{-\infty}^{\infty} \frac{\left(s+\psi\left(\varphi, \theta_{\mathbf{q}}\right)^{2} d s\right.}{\left(s-\psi_{+}\left(\varphi, \theta_{\mathbf{q}}\right)\right)^{2}\left(s-\psi_{-}\left(\varphi, \theta_{\mathbf{q}}\right)\right)^{2}+s^{2}},
$$

where we have defined

$\psi_{ \pm}\left(\varphi, \theta_{\mathbf{q}}\right) \equiv \frac{c_{ \pm}\left(\theta_{\mathbf{q}}\right)-v_{\rho} \cos \theta_{\mathbf{q}} \Xi\left(\varphi, \theta_{\mathbf{q}}\right)}{\Psi\left(1, \theta_{\mathbf{q}}\right)}$ and

$$
\psi\left(\varphi, \theta_{\mathbf{q}}\right) \equiv \frac{v_{\rho} \cos \theta_{\mathbf{q}}\left(\Xi\left(\varphi, \theta_{\mathbf{q}}\right)-1\right)}{\Psi\left(1, \theta_{\mathbf{q}}\right)}
$$

We will show in appendix (C) that, whenever the stability condition is satisfied, $\psi_{+}>0$ and $\psi_{-}<0$; we will make use of these facts later.

We also show in appendix C that this integral is equal to

$\int_{-\infty}^{\infty} \frac{\left(s+\psi\left(\varphi, \theta_{\mathbf{q}}\right)^{2} d s\right.}{\left(s-\psi_{+}\left(\varphi, \theta_{\mathbf{q}}\right)\right)^{2}\left(s-\psi_{-}\left(\varphi, \theta_{\mathbf{q}}\right)\right)^{2}+s^{2}}=\pi\left[1+\left(\frac{\gamma_{\rho}}{\gamma}\right)^{2} \frac{m^{2} \sin ^{2} \theta_{\mathbf{q}} \cos ^{2} \theta_{\mathbf{q}}}{\left\{\left(1+\sin ^{2} \theta_{\mathbf{q}}\right)^{2}-m^{2} \frac{\gamma_{\rho}}{\gamma}\left[\varpi-1+(\varpi-\varphi) \sin ^{2} \theta_{\mathbf{q}}\right] \cos ^{2} \theta_{\mathbf{q}}\right\}}\right]$.

Using (V.19), we obtain the expression for the equal- time correlation function:

$$
\begin{aligned}
C_{p p}(\mathbf{q}) & =\frac{D_{p}}{q \Psi\left(1, \theta_{\mathbf{q}}\right)}\left[1+\left(\frac{\gamma_{\rho}}{\gamma}\right)^{2} \frac{m^{2} \sin ^{2} \theta_{\mathbf{q}} \cos ^{2} \theta_{\mathbf{q}}}{D\left(m^{2}, \varpi, \varphi, \theta_{\mathbf{q}}\right)}\right] \\
& =\frac{D_{p}}{\gamma q\left(1+\sin ^{2} \theta_{\mathbf{q}}\right)}\left[1+\left(\frac{\gamma_{\rho}}{\gamma}\right)^{2} \frac{m^{2} \sin ^{2} \theta_{\mathbf{q}} \cos ^{2} \theta_{\mathbf{q}}}{D\left(m^{2}, \varpi, \varphi, \theta_{\mathbf{q}}\right)}\right],
\end{aligned}
$$

where we have defined

$$
D\left(m^{2}, \varpi, \varphi, \theta_{\mathbf{q}}\right)=\left[\left(1+\sin ^{2} \theta_{\mathbf{q}}\right)^{2}-m^{2} \frac{\gamma_{\rho}}{\gamma}\left[\varpi-1+(\varpi-\varphi) \sin ^{2} \theta_{\mathbf{q}}\right] \cos ^{2} \theta_{\mathbf{q}}\right]
$$

This expression is easily Fourier transformed in space to give the real space correlations $C_{p p}(\mathbf{r})$ of the polarization fluctuations:

$$
C_{p p}(\mathbf{r}) \equiv\left\langle p_{y}(\mathbf{r}+\mathbf{R}, t) p_{y}(\mathbf{R}, t)\right\rangle=\int \frac{d^{2} q}{(2 \pi)^{2}} e^{i \mathbf{q} \cdot \mathbf{r}} C_{p p}(\mathbf{q}) .
$$

Defining $\theta_{\mathbf{r}}$ as the angle between $\mathbf{r}$ and the $x$-axis, we can rewrite this as 


$$
C_{p p}(\mathbf{r})=\frac{D_{p}}{(2 \pi)^{2} \gamma} \int_{0}^{2 \pi} \frac{d \theta_{\mathbf{q}}}{1+\sin ^{2} \theta_{\mathbf{q}}}\left[1+\left(\frac{\gamma_{\rho}}{\gamma}\right)^{2} \frac{m^{2} \sin ^{2} \theta_{\mathbf{q}} \cos ^{2} \theta_{\mathbf{q}}}{D\left(m^{2}, \varpi, \varphi, \theta_{\mathbf{q}}\right)}\right] \int_{0}^{\infty} d q e^{i q r \cos \left(\theta_{\mathbf{q}}-\theta_{\mathbf{r}}\right)}
$$

The integral over $q$ is given by

$$
\begin{aligned}
& \int_{0}^{\infty} d q e^{i q r \cos \left(\theta_{\mathbf{q}}-\theta_{\mathbf{r}}\right)}=\pi \delta\left(r \cos \left(\theta_{\mathbf{q}}-\theta_{\mathbf{r}}\right)\right)+\mathcal{P}\left[\frac{1}{r \cos \left(\theta_{\mathbf{q}}-\theta_{\mathbf{r}}\right)}\right] \\
& \quad C_{p p}(\mathbf{r})=\frac{D_{p}}{2 \pi \gamma} \int_{0}^{2 \pi} \frac{d \theta_{\mathbf{q}}}{1+\sin ^{2} \theta_{\mathbf{q}}}\left[1+\left(\frac{\gamma_{\rho}}{\gamma}\right)^{2} \frac{m^{2} \sin ^{2} \theta_{\mathbf{q}} \cos ^{2} \theta_{\mathbf{q}}}{D\left(m^{2}, \varpi, \varphi, \theta_{\mathbf{q}}\right)}\right]\left(\delta\left(r \cos \left(\theta_{\mathbf{q}}-\theta_{\mathbf{r}}\right)\right)+\mathcal{P}\left[\frac{1}{\pi r \cos \left(\theta_{\mathbf{q}}-\theta_{\mathbf{r}}\right)}\right]\right) .
\end{aligned}
$$

The Cauchy principal value term in this expression is odd under the operation $\theta_{\mathbf{q}} \rightarrow \theta_{\mathbf{q}}+\pi$, while $\frac{1}{1+\sin ^{2} \theta_{\mathbf{q}}}$ is even under this operation; hence, the contribution to

integral from the Cauchy principal value term vanishes. We are therefore left with

$$
C_{p p}(\mathbf{r})=\frac{D_{p}}{4 \pi \gamma} \int_{0}^{2 \pi} \frac{d \theta_{\mathbf{q}}}{1+\sin ^{2} \theta_{\mathbf{q}}}\left[1+\left(\frac{\gamma_{\rho}}{\gamma}\right)^{2} \frac{m^{2} \sin ^{2} \theta_{\mathbf{q}} \cos ^{2} \theta_{\mathbf{q}}}{D\left(m^{2}, \varpi, \varphi, \theta_{\mathbf{q}}\right)}\right] \delta\left(r \cos \left(\theta_{\mathbf{q}}-\theta_{\mathbf{r}}\right)\right)
$$

Using

$$
\delta\left(r \cos \left(\theta_{\mathbf{q}}-\theta_{\mathbf{r}}\right)\right)=\frac{\delta\left(\theta_{\mathbf{q}}-\theta_{\mathbf{r}}+\pi / 2\right)+\delta\left(\theta_{\mathbf{q}}-\theta_{\mathbf{r}}-\pi / 2\right)}{r\left|\left(\frac{\partial \cos \left(\theta_{\mathbf{q}}-\theta_{\mathbf{r}}\right)}{\partial \theta_{\mathbf{q}}}\right)_{\theta_{\mathbf{q}}=\theta_{\mathbf{r}} \pm \pi / 2}\right|}=\frac{\delta\left(\theta_{\mathbf{q}}-\theta_{\mathbf{r}}+\pi / 2\right)+\delta\left(\theta_{\mathbf{q}}-\theta_{\mathbf{r}}-\pi / 2\right)}{r}
$$
tity

where the first equality follows from the familiar iden-

$$
\delta(f(x))=\sum_{x_{0}} \delta\left(x-x_{0}\right) /\left|f^{\prime}\left(x_{0}\right)\right|
$$

where $\left\{x_{0}\right\}$ are the roots of $f(x), \mathrm{V} .25$ becomes

$$
\begin{aligned}
C_{p p}(\mathbf{r})= & \frac{D_{p}}{4 \pi \gamma r} \int_{0}^{2 \pi} \frac{d \theta_{\mathbf{q}}}{1+\sin ^{2} \theta_{\mathbf{q}}}\left[1+\left(\frac{\gamma_{\rho}}{\gamma}\right)^{2} \frac{m^{2} \sin ^{2} \theta_{\mathbf{q}} \cos ^{2} \theta_{\mathbf{q}}}{D\left(m^{2}, \varpi, \varphi, \theta_{\mathbf{q}}\right)}\right]\left(\delta\left(\theta_{\mathbf{q}}-\theta_{\mathbf{r}}+\pi / 2\right)+\delta\left(\theta_{\mathbf{q}}-\theta_{\mathbf{r}}-\pi / 2\right)\right) \\
& =\frac{D_{p} r}{2 \pi \gamma\left(r^{2}+x^{2}\right)}\left[1+\left(\frac{\gamma_{\rho}}{\gamma}\right)^{2} \frac{m^{2} y^{2} x^{2}}{\left\{\left(r^{2}+x^{2}\right)^{2}-m^{2} \frac{\gamma_{\rho}}{\gamma} y^{2}\left[r^{2}(\varpi-1)+(\varpi-\varphi) x^{2}\right]\right\}}\right]
\end{aligned}
$$

We now turn to the equal-time density correlations. Using (V.6), we have

$$
\begin{aligned}
& C_{\rho \rho}(\mathbf{q}) \equiv\left\langle|\rho(\mathbf{q}, t)|^{2}\right\rangle \\
& =\int_{-\infty}^{\infty} \frac{d \omega}{2 \pi}\left\langle|\rho(\mathbf{q}, \omega)|^{2}\right\rangle=\frac{D_{\rho} \rho_{c}^{2} v_{\rho}^{2} q^{2} \sin ^{2} \theta_{\mathbf{q}}}{\pi} \int_{-\infty}^{\infty} \frac{d \omega}{\left(\omega-c_{+}\left(\theta_{\mathbf{q}}\right) q\right)^{2}\left(\omega-c_{-}\left(\theta_{\mathbf{q}}\right) q\right)^{2}+\left(\omega \Psi\left(1, \theta_{\mathbf{q}}\right)-v_{\rho} q \Psi\left(\varphi, \theta_{\mathbf{q}}\right) \cos \theta_{\mathbf{q}}\right)^{2} q^{2}}
\end{aligned}
$$




$$
C_{\rho \rho}(\mathbf{q})=\frac{D_{p} \rho_{c}^{2} v_{\rho}^{2} q^{2} \sin ^{2} \theta_{\mathbf{q}}}{2 i \pi\left[\gamma q\left(1+\sin ^{2} \theta_{\mathbf{q}}\right)\right]^{3}}\left(I_{\rho}^{-}-I_{\rho}^{+}\right),
$$

where we have defined

$$
I_{\rho}^{ \pm} \equiv \lim _{\epsilon \rightarrow 0} \int_{-\infty}^{\infty} \frac{d s}{\left(s-i \epsilon-\psi_{+}\left(\theta_{\mathbf{q}}\right)\right)\left(s-i \epsilon-\psi_{-}\left(\theta_{\mathbf{q}}\right)\right) \pm i(s-i \epsilon)} \frac{1}{s-i \epsilon}
$$

where we have introduced the small parameter $\epsilon$ to regularize the integral.

We can now do both integrals by the usual complex contour techniques. Note that, unlike the integral for the polarization correlation function $C_{p p}$, the integrand in V.31 converges rapidly enough at infinity that the infinite semicircle needed to close the path of integration contributes nothing to the integral. Note also that the poles of $I_{\rho}^{ \pm}$are those of $I_{ \pm}$considered earlier, plus one more pole at $s=i \epsilon$.

If we choose $\epsilon>0$, then we can close the integral for $I_{\rho}^{+}$in the upper half plane, picking up the pole at $s=i \epsilon$. This gives

$$
I_{\rho}^{+}=\frac{2 i \pi}{\psi_{+} \psi_{-}}
$$

where we have taken the limit $\epsilon \rightarrow 0$.

On the other hand, we can close the contour for $I_{\rho}^{-}$in the lower half plane, finding (for $\epsilon>0$ ) no poles at all. This implies

$$
I_{\rho}^{-}=0
$$

Inserting $(\mathrm{V} .32$ and $\mathrm{V} .33$ into $(\mathrm{V} .30)$, we obtain

$$
C_{\rho \rho}(\mathbf{q})=-\frac{D_{p} \rho_{c}^{2} v_{\rho}^{2} q^{2} \sin ^{2} \theta_{\mathbf{q}}}{\left[\gamma q\left(1+\sin ^{2} \theta_{\mathbf{q}}\right)\right]^{3} \psi_{+} \psi_{-}} .
$$

It is straightforward to check that, had we chosen $\epsilon<0$, we would have gotten the same answer, although now with $I_{\rho}^{+}=0$ and $I_{\rho}^{-}=-\frac{2 i \pi}{\psi_{+} \psi_{-}}$.

It is an equally straightforward algebraic exercise, using the definitions $\sqrt{\mathrm{V} .17}$ of $\psi_{ \pm}$and our expressions $\sqrt{\mathrm{V} .4}$ for $c_{ \pm}\left(\theta_{\mathbf{q}}\right)$ to show that

$$
\psi_{+} \psi_{-}=-\frac{D\left(m^{2}, \varpi, \varphi, \theta_{\mathbf{q}}\right) v_{\rho}^{2} \gamma^{2} \sin ^{2} \theta_{\mathbf{q}}}{m^{2}\left[\Psi\left(1, \theta_{\mathbf{q}}\right)\right]^{4}} .
$$

Using this in $\mathrm{V} .34$ gives

$$
C_{\rho \rho}(\mathbf{q})=\frac{D_{p} \rho_{c}^{2} m^{2}\left(1+\sin ^{2} \theta_{\mathbf{q}}\right)}{\gamma q D\left(m^{2}, \varpi, \varphi, \theta_{\mathbf{q}}\right)} .
$$

The above expression can be easily Fourier transformed in space to obtain the real space density correlations $C_{\rho \rho}(\mathbf{r})$ just as we did above to obtain $C_{p p}(\mathbf{r})$. We thereby obtain:

$$
C_{\rho \rho}(\mathbf{r}) \equiv\langle\delta \rho(\mathbf{r}+\mathbf{R}, t) \delta \rho(\mathbf{R}, t)\rangle=\frac{D_{p} \rho_{c}^{2} m^{2}}{2 \pi \gamma} \frac{r\left(r^{2}+x^{2}\right)}{\left\{\left(r^{2}+x^{2}\right)^{2}-m^{2} \frac{\gamma_{\rho}}{\gamma} y^{2}\left[r^{2}(\varpi-1)+(\varpi-\varphi) x^{2}\right]\right\}} .
$$




$$
\begin{aligned}
C_{p \rho}(\mathbf{q}) \equiv\left\langle\rho(\mathbf{q}) p_{y}(-\mathbf{q})\right\rangle & =\int_{-\infty}^{\infty} \frac{d \omega}{2 \pi}\left\langle\rho(\mathbf{q}, \omega) p_{y}(-\mathbf{q},-\omega)\right\rangle \\
& =\int_{-\infty}^{\infty} \frac{d \omega}{2 \pi} \frac{2 D_{p} \rho_{c} v_{\rho} q \sin \theta_{\mathbf{q}}\left(\omega-v_{\rho} q \cos \theta_{\mathbf{q}}\right)}{\left(\omega-c_{+}\left(\theta_{\mathbf{q}}\right) q\right)^{2}\left(\omega-c_{-}\left(\theta_{\mathbf{q}}\right) q\right)^{2}+\left(\omega \Psi\left(1, \theta_{\mathbf{q}}\right)-v_{\rho} q \Psi\left(\varphi, \theta_{\mathbf{q}}\right) \cos \theta_{\mathbf{q}}\right)^{2} q^{2}} \\
& =\frac{D_{p} \rho_{c} v_{\rho} q \sin \theta_{\mathbf{q}}}{\pi \gamma^{2} q^{2}\left(1+\sin ^{2} \theta_{\mathbf{q}}\right)^{2}} \int_{-\infty}^{\infty} \frac{\left(s+\psi\left(\varphi, \theta_{\mathbf{q}}\right)\right) d s}{\left(s-\psi_{+}\right)^{2}\left(s-\psi_{-}\right)^{2}+s^{2}} .
\end{aligned}
$$

From equations $(\overline{\mathrm{C} 9})$ and $(\mathrm{C} 10)$, we can easily show that

$$
\int_{-\infty}^{\infty} \frac{\left(s+\psi\left(\varphi, \theta_{\mathbf{q}}\right)\right) d s}{\left(s-\psi_{+}\right)^{2}\left(s-\psi_{-}\right)^{2}+s^{2}}=I_{2}+\psi\left(\varphi, \theta_{\mathbf{q}}\right) I_{3}
$$

where

$$
I_{2}=0, \text { and } I_{3}=-\frac{\pi}{\psi_{+} \psi_{-}} .
$$

Using eqs. V.40 and (V.38), we obtain

$$
C_{p \rho}(\mathbf{q})=-\frac{D_{p} \rho_{c} \sin \theta_{\mathbf{q}} \cos \theta_{\mathbf{q}} m^{2} \gamma_{\rho}}{\gamma^{2} q D\left(m^{2}, \varpi, \varphi, \theta_{\mathbf{q}}\right)}
$$

Fourier transformation of the above expression gives the real space cross correlation, given by

$$
C_{p \rho}(\mathbf{r})=-\frac{D_{p} \rho_{c} m^{2} \gamma_{\rho}}{2 \pi \gamma^{2}} \frac{x y r}{\left\{\left(r^{2}+x^{2}\right)^{2}-m^{2} \frac{\gamma_{\rho}}{\gamma} y^{2}\left[r^{2}(\varpi-1)+(\varpi-\varphi) x^{2}\right]\right\}} .
$$

To summarize, we have shown that over a finite range of the model parameters, the linearized model displays long range orientational order. We argue below that this linear theory prediction is asymptotically exact, in contrast to the well-known Toner-Tu model for dry active matter, for which nonlinear effects are essential for the existence of the long range order [13, 14].

\section{GIANT NUMBER FLUCTUATIONS AND THEIR SHAPE DEPENDENCE}

We will show in this section that the long-ranged density correlations found in equation (V.37) of the previous section lead to "giant number fluctuations" 48] 15, 17,
19. These can be defined as follows:

Consider a "counting box", defined as a rectangular area $A=L_{x} \times L_{y}$, and define the aspect ratio $\alpha_{A}=L_{x} / L_{y}$. Our experiment will consist of counting the number of active particles in this box. The mean number of particles $\bar{N}$ in the box is, of course, given by

$$
\bar{N}=L_{x} L_{y} \rho_{0}=\alpha_{A} L_{y}^{2} \rho_{0} .
$$

The total number fluctuation $\delta N \equiv N-\bar{N}$ in the area $A$ is

Thence,

$$
\delta N=\int d^{2} r \delta \rho(\mathbf{r}, t)
$$

$$
\begin{aligned}
& \left\langle(\delta N)^{2}\right\rangle=\int_{0}^{L_{x}} d x \int_{0}^{L_{y}} d y \int_{0}^{L_{x}} d x^{\prime} \int_{0}^{L_{y}} d y^{\prime}\left\langle\rho(\mathbf{r}, t) \rho\left(\mathbf{r}^{\prime}, t\right)\right\rangle=\int_{0}^{L_{x}} d x \int_{0}^{L_{y}} d y \int_{0}^{L_{x}} d x^{\prime} \int_{0}^{L_{y}} d y^{\prime} C_{\rho \rho}\left(\mathbf{r}-\mathbf{r}^{\prime}\right) \\
& =\frac{D_{p} \rho_{c}^{2} m^{2}}{2 \pi \gamma} \int_{0}^{L_{x}} d x \int_{0}^{L_{y}} d y \int_{0}^{L_{y}} d y^{\prime} \int_{0}^{L_{x}} d x^{\prime} \frac{\left|\mathbf{r}-\mathbf{r}^{\prime}\right|}{\left(\mathbf{r}-\mathbf{r}^{\prime}\right)^{2}+\left(x-x^{\prime}\right)^{2}} \\
& \times\left[1-\frac{\left(v_{p}-v_{\rho}\right)\left(y-y^{\prime}\right)^{2}}{\left(\mathbf{r}-\mathbf{r}^{\prime}\right)^{2}+\left(x-x^{\prime}\right)^{2}} \frac{\gamma_{\rho} v_{\rho}}{\gamma c_{0}^{2}}-\left(\frac{v_{\rho} \gamma_{\rho}}{c_{0} \gamma}\right)^{2} \frac{\left(y-y^{\prime}\right)^{2}\left(x-x^{\prime}\right)^{2}}{\left\{\left(\mathbf{r}-\mathbf{r}^{\prime}\right)^{2}+\left(x-x^{\prime}\right)^{2}\right\}^{2}}\right]^{-1}
\end{aligned}
$$

We consider the limits $\alpha_{A} \gg 1$ and $\alpha_{A} \ll 1$.

Consider first the limit $L_{x} \gg L_{y}$, i.e., $\alpha_{A} \gg 1$. Now 
consider the integral over $x^{\prime}$ for fixed $\mathbf{r}=(x, y)$ in this limit. Note first that in this limit, for most of the range of integration over $x, x \gg L_{y}$. For such values of $x$, we can split the integral over $x^{\prime}$ into three parts:

$$
\begin{aligned}
& \int_{0}^{L_{x}} d x^{\prime} \frac{\left|\mathbf{r}-\mathbf{r}^{\prime}\right|}{\left(\mathbf{r}-\mathbf{r}^{\prime}\right)^{2}+\left(x-x^{\prime}\right)^{2}} \\
& \times\left[1-\frac{\left(v_{p}-v_{\rho}\right)\left(y-y^{\prime}\right)^{2}}{\left(\mathbf{r}-\mathbf{r}^{\prime}\right)^{2}+\left(x-x^{\prime}\right)^{2}} \frac{\gamma_{\rho} v_{\rho}}{\gamma c_{0}^{2}}-\left(\frac{v_{\rho} \gamma_{\rho}}{c_{0} \gamma}\right)^{2} \frac{\left(y-y^{\prime}\right)^{2}\left(x-x^{\prime}\right)^{2}}{\left\{\left(\mathbf{r}-\mathbf{r}^{\prime}\right)^{2}+\left(x-x^{\prime}\right)^{2}\right\}^{2}}\right]^{-1}=I_{1}+I_{2}+I_{3},
\end{aligned}
$$

where we have defined

$$
\begin{aligned}
I_{1} \equiv & \int_{0}^{x-C L_{y}} d x^{\prime} \frac{\left|\mathbf{r}-\mathbf{r}^{\prime}\right|}{\left(\mathbf{r}-\mathbf{r}^{\prime}\right)^{2}+\left(x-x^{\prime}\right)^{2}} \\
& \times\left[1-\frac{\left(v_{p}-v_{\rho}\right)\left(y-y^{\prime}\right)^{2}}{\left(\mathbf{r}-\mathbf{r}^{\prime}\right)^{2}+\left(x-x^{\prime}\right)^{2}} \frac{\gamma_{\rho} v_{\rho}}{\gamma c_{0}^{2}}-\left(\frac{v_{\rho} \gamma_{\rho}}{c_{0} \gamma}\right)^{2} \frac{\left(y-y^{\prime}\right)^{2}\left(x-x^{\prime}\right)^{2}}{\left\{\left(\mathbf{r}-\mathbf{r}^{\prime}\right)^{2}+\left(x-x^{\prime}\right)^{2}\right\}^{2}}\right]^{-1}, \\
I_{2} \equiv & \int_{x-C L_{y}}^{x+C L_{y}} d x^{\prime} \frac{\left|\mathbf{r}-\mathbf{r}^{\prime}\right|}{\left(\mathbf{r}-\mathbf{r}^{\prime}\right)^{2}+\left(x-x^{\prime}\right)^{2}} \\
& \times\left[1-\frac{\left(v_{p}-v_{\rho}\right)\left(y-y^{\prime}\right)^{2}}{\left(\mathbf{r}-\mathbf{r}^{\prime}\right)^{2}+\left(x-x^{\prime}\right)^{2}} \frac{\gamma_{\rho} v_{\rho}}{\gamma c_{0}^{2}}-\left(\frac{v_{\rho} \gamma_{\rho}}{c_{0} \gamma}\right)^{2} \frac{\left(y-y^{\prime}\right)^{2}\left(x-x^{\prime}\right)^{2}}{\left\{\left(\mathbf{r}-\mathbf{r}^{\prime}\right)^{2}+\left(x-x^{\prime}\right)^{2}\right\}^{2}}\right]^{-1}, \\
I_{3} \equiv & \int_{x+C L_{y}}^{L_{x}} d x^{\prime} \frac{\left|\mathbf{r}-\mathbf{r}^{\prime}\right|}{\left(\mathbf{r}-\mathbf{r}^{\prime}\right)^{2}+\left(x-x^{\prime}\right)^{2}} \\
& \times\left[1-\frac{\left(v_{p}-v_{\rho}\right)\left(y-y^{\prime}\right)^{2}}{\left(\mathbf{r}-\mathbf{r}^{\prime}\right)^{2}+\left(x-x^{\prime}\right)^{2}} \frac{\gamma_{\rho} v_{\rho}}{\gamma c_{0}^{2}}-\left(\frac{v_{\rho} \gamma_{\rho}}{c_{0} \gamma}\right)^{2} \frac{\left(y-y^{\prime}\right)^{2}\left(x-x^{\prime}\right)^{2}}{\left\{\left(\mathbf{r}-\mathbf{r}^{\prime}\right)^{2}+\left(x-x^{\prime}\right)^{2}\right\}^{2}}\right]^{-1},
\end{aligned}
$$

where $C$ is an arbitrary constant enough larger than 1 that we can safely neglect terms of $\mathcal{O}\left(\frac{1}{C}\right)$. With this choice, throughout the region of integration for $I_{1}, x-x^{\prime} \gg L_{y}$. Therefore, throughout this region, $\left|y-y^{\prime}\right| \ll x-x^{\prime}$ (since $\left|y-y^{\prime}\right|$ is always $<L_{y}$ ). This gives $\left(\frac{y-y^{\prime}}{x-x^{\prime}}\right)^{2} \ll 1$. Making this approximation for $I_{1}$, our expression for it reduces to

$$
I_{1} \approx \frac{1}{2} \int_{0}^{x-C L_{y}} \frac{d x^{\prime}}{x-x^{\prime}} .
$$

Performing this elementary integral gives

$$
I_{1}=\frac{1}{2} \ln \left(\frac{x}{C L_{y}}\right)=\frac{1}{2} \ln \left(\frac{x}{L_{y}}\right)-\frac{1}{2} \ln C .
$$

Virtually identical reasoning can be applied to $I_{3}$, giving the result

$$
\begin{aligned}
I_{2}= & \int_{-C L_{y}}^{C L_{y}} d x^{\prime \prime} \frac{\sqrt{x^{\prime \prime 2}+\left(y-y^{\prime}\right)^{2}}}{\left(y-y^{\prime}\right)^{2}+2 x^{\prime \prime 2}} \\
& \left.\times\left[1-\frac{\gamma_{\rho} v_{\rho}}{\gamma c_{0}^{2}} \frac{\left(v_{p}-v_{\rho}\right)\left(y-y^{\prime}\right)^{2}}{\left(y-y^{\prime}\right)^{2}+2 x^{\prime \prime 2}}-\left(\frac{v_{\rho} \gamma_{\rho}}{c_{0} \gamma}\right)^{2} \frac{\left(y-y^{\prime}\right)^{2} x^{\prime \prime 2}}{\left[\left(y-y^{\prime}\right)^{2}+2 x^{\prime \prime 2}\right.}\right]^{2}\right]^{-1}
\end{aligned}
$$

For $I_{2}$, a simple shift of variables of integration $x^{\prime}=$ $x+x^{\prime \prime}$ shows that $I_{2}$ is independent of $x$ : 
our expression VI.4, and using that result in our expression VI.3 for $\left\langle(\delta N)^{2}\right\rangle$, we obtain

$$
\begin{aligned}
& \left\langle(\delta N)^{2}\right\rangle \approx \frac{D_{p} \rho_{c}^{2} m^{2}}{2 \pi \gamma}\left\{\frac{1}{2} \int_{0}^{L_{x}} d x \int_{0}^{L_{y}} d y \int_{0}^{L_{y}} d y^{\prime}\left(\ln \left(\frac{x}{L_{y}}\right)+\ln \left(\frac{L_{x}-x}{L_{y}}\right)-2 \ln C\right)\right. \\
& \left.+\int_{0}^{L_{x}} d x \int_{0}^{L_{y}} d y \int_{0}^{L_{y}} d y^{\prime} \int_{-C L_{y}}^{C L_{y}} d x^{\prime \prime} \frac{\sqrt{x^{\prime \prime 2}+\left(y-y^{\prime}\right)^{2}}}{\left[\left(y-y^{\prime}\right)^{2}+2 x^{\prime \prime 2}\right]} \times\left[1-\frac{\gamma_{\rho} v_{\rho}}{\gamma c_{0}^{2}} \frac{\left(v_{p}-v_{\rho}\right)\left(y-y^{\prime}\right)^{2}}{\left[\left(y-y^{\prime}\right)^{2}+2 x^{\prime \prime 2}\right]}-\left(\frac{v_{\rho} \gamma_{\rho}}{c_{0} \gamma}\right)^{2} \frac{\left(y-y^{\prime}\right)^{2} x^{\prime \prime 2}}{\left[\left(y-y^{\prime}\right)^{2}+2 x^{\prime \prime 2}\right]^{2}}\right]^{-1}\right\}
\end{aligned}
$$

The integrals over $y$ and $y^{\prime}$ in the (triple) integral in the first line of this expression trivially give a factor of
$L_{y}^{2}$, since the integrand is independent of $y$ and $y^{\prime}$. The remaining integral over $x$ is elementary. The net result is

$$
\frac{1}{2} \int_{0}^{L_{x}} d x \int_{0}^{L_{y}} d y \int_{0}^{L_{y}} d y^{\prime}\left(\ln \left(\frac{x}{L_{y}}\right)+\ln \left(\frac{L_{x}-x}{L_{y}}\right)-2 \ln C\right)=L_{x} L_{y}^{2}\left(\ln \left(\frac{L_{x}}{C L_{y}}\right)-1\right)
$$

The $x$ integral in the remaining (quadruple) integral which appears on the second line in equation VI.12 can be done immediately, since the integrand is independent of $x$, yielding a factor of $L_{x}$. The remaining triple integral over $y, y^{\prime}$, and $x^{\prime \prime}$ can be done by changing variables of integration to new, rescaled variables $u_{x}, u_{y}$, and $u_{y}^{\prime}$ via

$$
y \equiv L_{y} u_{y} \quad, \quad y^{\prime} \equiv L_{y} u_{y}^{\prime} \quad, \quad x^{\prime \prime} \equiv L_{y} u_{x} .
$$

This gives

$$
\begin{aligned}
\int_{0}^{L_{x}} d x \int_{0}^{L_{y}} d y \int_{0}^{L_{y}} d y^{\prime} \int_{-C L_{y}}^{C L_{y}} d x^{\prime \prime} & \frac{\sqrt{x^{\prime \prime 2}+\left(y-y^{\prime}\right)^{2}}}{\left[\left(y-y^{\prime}\right)^{2}+2 x^{\prime \prime 2}\right]} \times\left[1-\frac{\gamma_{\rho} v_{\rho}}{\gamma c_{0}^{2}} \frac{\left(v_{p}-v_{\rho}\right)\left(y-y^{\prime}\right)^{2}}{\left[\left(y-y^{\prime}\right)^{2}+2 x^{\prime \prime 2}\right]}-\left(\frac{v_{\rho} \gamma_{\rho}}{c_{0} \gamma}\right)^{2} \frac{\left(y-y^{\prime}\right)^{2} x^{\prime \prime 2}}{\left[\left(y-y^{\prime}\right)^{2}+2 x^{\prime \prime 2}\right]^{2}}\right]^{-1} \\
& =C^{\prime} L_{x} L_{y}^{2}
\end{aligned}
$$

where

$$
\begin{aligned}
C^{\prime} \equiv & \int_{0}^{1} d u_{y} \int_{0}^{1} d u_{y}^{\prime} \int_{-C}^{C} d u_{x} \frac{\sqrt{u_{x}^{2}+\left(u_{y}-u_{y}^{\prime}\right)^{2}}}{\left(u_{y}-u_{y}^{\prime}\right)^{2}+2 u_{x}^{2}} \\
& {\left[1-\frac{\gamma_{\rho} v_{\rho}}{\gamma c_{0}^{2}} \frac{\left(v_{p}-v_{\rho}\right)\left(u_{y}-u_{y}^{\prime}\right)^{2}}{2 u_{x}^{2}+\left(u_{y}-u_{y}^{\prime}\right)^{2}}-\left(\frac{v_{\rho} \gamma_{\rho}}{c_{0} \gamma}\right)^{2} \frac{\left(u_{y}-u_{y}^{\prime}\right)^{2} u_{x}^{2}}{\left[2 u_{x}^{2}+\left(u_{y}-u_{y}^{\prime}\right)^{2}\right]^{2}}\right]^{-1} }
\end{aligned}
$$

is an $\mathcal{O}(1)$ constant. Comparing (VI.13) and (VI.15), we see that the first line of VI.12) actually dominates the second in the large aspect ratio limit $L_{x} \gg L_{y}$ that we are considering here. Therefore, we obtain, in the limit of large aspect ratio $\left(\alpha_{A} \gg 1\right)$ : 


$$
\sqrt{\left\langle(\delta N)^{2}\right\rangle} \approx \frac{\rho_{c} m \sqrt{D_{p}}}{\sqrt{2 \pi \gamma}} \sqrt{L_{x} L_{y}^{2} \ln \left(\frac{L_{x}}{L_{y}}\right)} \approx \frac{\rho_{c} m \sqrt{D_{p}}}{\sqrt{2 \pi \gamma}} L_{y}^{3 / 2} \sqrt{\alpha_{A} \ln \alpha_{A}}
$$

This expression VI.17 can be rewritten in terms of the mean particle number $\bar{N}$ in the same area $A$, which is given by

$$
\bar{N}=L_{x} L_{y} \rho_{0}=\alpha_{A} L_{y}^{2} \rho_{0}
$$

This gives

$$
L_{y}=\left(\bar{N} /\left(\alpha_{A} \rho_{0}\right)\right)^{1 / 2}
$$

Using this result (VI.19) in our expression (VI.17) for the variance $(\Delta N)^{2}$ of the number fluctuations gives

$\Delta N=\sqrt{\left\langle(\delta N)^{2}\right\rangle}=\left(\frac{\rho_{c} m}{\rho_{0}^{3 / 4}}\right)\left(\sqrt{\frac{D_{p}}{2 \pi \gamma}}\right)\left(\frac{\bar{N}^{3 / 4} \sqrt{\ln \alpha_{A}}}{\alpha_{A}^{1 / 4}}\right)$

Three points should be noted about this result:

1) The number fluctuations are giant; that is, they grow much more rapidly with $\bar{N}$ than the usual $\sqrt{\bar{N}}$ "law of large numbers" fluctuations, which are found in almost all systems, and, in particular, in most equilibrium systems away from fixed points 49 . Specifically, we have $\Delta N \propto \bar{N}^{3 / 4}$. Note also that the exponent $3 / 4$ is very close to the exponent .8 found in the experiments of [27].

2) The size of the number fluctuations depend not only on the mean number $\bar{N}$, but also on the shape (i.e., on the aspect ratio $\alpha_{A}$ ).
3) The number fluctuations $\Delta N$ are a monotonically decreasing function of the aspect ratio $\alpha_{A}$ in this range of $\alpha_{A} \gg 1$.

We analyze giant number fluctuations in the opposite regime of $L_{y} \gg L_{x}$, i.e., small aspect ratio $\alpha_{A} \ll 1$ in Appendix E. As shown there, in this regime of small aspect ratio $\alpha_{A}, \Delta N$ is a monotonically increasing function of $\alpha_{A}$. Recall that in the opposite limit of $\alpha_{A} \gg 1$, eqn VI.20, we found that $\Delta N$ is a monotonically decreas$\mathrm{ing}$ function of $\alpha_{A}$. Hence, the maximum value of $\Delta N$ for a given mean number of particles $\bar{N}$ will occur when $\alpha_{A} \sim 1$; i.e., for a roughly square counting box.

In contrast to our above results on giant number fluctuations, Ref. 28] did not report any giant number fluctuations in their experiment. We believe this is due to the fact that in their experiment, the density fluctuations were probed at length scales larger than the height of the passive fluid over the active layer, which is outside the regime of validity of our theory.

\section{BULK VELOCITY FLUCTUATIONS}

We can use the relations (III.38) and III.39 between the bulk fluid velocity field and the surface velocity, and our boundary condition (III.14) for that surface velocity, to obtain expressions for the bulk velocity correlations in terms of the polarization $\hat{\mathbf{p}}$ and density $\rho$ correlation functions. This gives, to the lowest order in gradients

$$
\begin{aligned}
& \left\langle v_{i}\left(\mathbf{r}_{\|}, z\right) v_{j}\left(\mathbf{r}_{\|}, z\right)\right\rangle=v_{0}^{2} \int \frac{d^{2} q}{(2 \pi)^{2}} C_{p p}(\mathbf{q}) e^{-2 q z}\left[\delta_{i y} \delta_{j y}-\frac{q_{y} z}{q}\left(\delta_{i y} q_{j}+\delta_{j y} q_{i}\right)+q_{y}^{2} z^{2}\left(\frac{q_{i} q_{j}}{q^{2}}+\delta_{i z} \delta_{i z}\right)\right] \\
& +v_{0} v_{a}^{\prime} \int \frac{d^{2} q}{(2 \pi)^{2}} C_{p \rho}(\mathbf{q}) e^{-2 q z}\left[\delta_{i y} \delta_{j x}+\delta_{i x} \delta_{j y}-z\left(\frac{q_{y}}{q} \delta_{i x} q_{j}+\frac{q_{y}}{q} \delta_{j x} q_{i}+\frac{q_{x}}{q} \delta_{j y} q_{i}+\frac{q_{x}}{q} \delta_{i y} q_{j}\right)+2 q_{x} q_{y} z^{2}\left(\frac{q_{i} q_{j}}{q^{2}}+\delta_{i z} \delta_{i z}\right)\right] \\
& +v_{a}^{\prime 2} \int \frac{d^{2} q}{(2 \pi)^{2}} C_{\rho \rho}(\mathbf{q}) e^{-2 q z}\left[\delta_{i x} \delta_{j x}-\frac{q_{x} z}{q}\left(\delta_{i x} q_{j}+\delta_{j x} q_{i}\right)+q_{x}^{2} z^{2}\left(\frac{q_{i} q_{j}}{q^{2}}+\delta_{i z} \delta_{i z}\right)\right]
\end{aligned}
$$

The second $\left(\delta_{i y} q_{j}\right)$ term in the first integral is odd in $q_{x}$ if $j=x$. Since $C_{p p}(\mathbf{q}) e^{-2 q z}$ and $q_{y}$ are even in $q_{x}$, the integral of this term vanishes when $i=x$. Hence, we can replace this term by $\delta_{i y} \delta_{j y} q_{y}$. Similar arguments imply that we can also replace the third $\left(\delta_{j y} q_{i}\right)$ term with $\delta_{i y} \delta_{j y} q_{y}$.
Similarly we can replace $\left(\delta_{i x} q_{j}\right)$ with $\delta_{i x} \delta_{j x} q_{x}$, and $\left(\delta_{j x} q_{i}\right)$ with $\delta_{i x} \delta_{j x} q_{x}$ in the third integral. In the second integral $\left(\delta_{i x} q_{j}\right)$ can be replaced with $\delta_{i x} \delta_{j y} q_{y},\left(\delta_{j x} q_{i}\right)$ with $\delta_{j x} \delta_{i y} q_{y},\left(\delta_{j y} q_{i}\right)$ with $\delta_{i x} \delta_{j y} q_{x}$, and $\left(\delta_{i y} q_{j}\right)$ with $\delta_{i y} \delta_{j x} q_{x}$ respectively. Thus, we can rewrite (VII.1) as 


$$
\begin{aligned}
\left\langle v_{i}\left(\mathbf{r}_{\|}, z\right) v_{j}\left(\mathbf{r}_{\|}, z\right)\right\rangle= & v_{0}^{2} \int \frac{d^{2} q}{(2 \pi)^{2}} C_{p p}(\mathbf{q}) e^{-2 q z}\left[\delta_{i y} \delta_{j y}\left(1-\frac{2 q_{y}^{2} z}{q}\right)+q_{y}^{2} z^{2}\left(\frac{q_{i} q_{j}}{q^{2}}+\delta_{i z} \delta_{j z}\right)\right] \\
& +v_{0} v_{a}^{\prime} \int \frac{d^{2} q}{(2 \pi)^{2}} C_{p \rho}(\mathbf{q}) e^{-2 q z}\left[\left(\delta_{i y} \delta_{j x}+\delta_{i x} \delta_{j y}\right)(1-z q)+2 z^{2} q_{x} q_{y}\left(\frac{q_{i} q_{j}}{q^{2}}+\delta_{i z} \delta_{j z}\right)\right] \\
& +v_{a}^{\prime 2} \int \frac{d^{2} q}{(2 \pi)^{2}} C_{\rho \rho}(\mathbf{q}) e^{-2 q z}\left[\delta_{i x} \delta_{j x}\left(1-\frac{2 q_{x}^{2} z}{q}\right)+q_{x}^{2} z^{2}\left(\frac{q_{i} q_{j}}{q^{2}}+\delta_{i z} \delta_{j z}\right)\right] .
\end{aligned}
$$

We also note that the $\frac{q_{i} q_{j}}{q^{2}}$ term in the first integral is also odd in $q_{x}$ unless $i=j$, because, if $i \neq j$, then one, and only one, of the indices $(i, j)$ must be $x$. Hence, its integral also vanishes if $i \neq j$. Identical reasoning implies that the third integral also vanishes for $i \neq j$. This makes the first and third integrals diagonal.

The second integral is non-zero only when $i=x, j=y$, or $i=y, j=x$. This is explicit for the first term, since that term is proportional to $\delta_{i y} \delta_{j x}+\delta_{i x} \delta_{j y}$. To see that it is also true for the second term (i.e., the $z^{2} q_{x} q_{y}$ term), note that term is odd in at least one of $q_{x}$ or $q_{y}$ unless $i=x, j=y$, or $i=y, j=x$, and will therefore integrate to zero otherwise.

In light of these observations, we can rewrite our expression VII.2 for the velocity correlations as

$\left\langle v_{i}\left(\mathbf{r}_{\|}, z\right) v_{j}\left(\mathbf{r}_{\|}, z\right)\right\rangle=v_{0}^{2} \Pi_{i j}^{(1)}(z)+v_{0} v_{a}^{\prime} \Pi_{i j}^{(2)}(z)+v_{a}^{\prime 2} \Pi_{i j}^{(3)}(z)$, where we have defined

$$
\begin{gathered}
\Pi_{i j}^{(1)}(z) \equiv \int \frac{d^{2} q}{(2 \pi)^{2}} C_{p p}(\mathbf{q}) e^{-2 q z}\left[\delta_{i y} \delta_{j y}\left(1-\frac{2 q_{y}^{2} z}{q}\right)+q_{y}^{2} z^{2}\left(\frac{q_{i} q_{j}}{q^{2}}+\delta_{i z} \delta_{j z}\right)\right] \\
\Pi_{i j}^{(2)}(z) \equiv \int \frac{d^{2} q}{(2 \pi)^{2}} C_{p \rho}(\mathbf{q}) e^{-2 q z}\left[1-z q+\frac{2 z^{2} q_{x}^{2} q_{y}^{2}}{q^{2}}\right]\left(\delta_{i y} \delta_{j x}+\delta_{i x} \delta_{j y}\right) \\
\Pi_{i j}^{(3)}(z) \equiv \int \frac{d^{2} q}{(2 \pi)^{2}} C_{\rho \rho}(\mathbf{q}) e^{-2 q z}\left[\delta_{i x} \delta_{j x}\left(1-\frac{2 q_{x}^{2} z}{q}\right)+q_{x}^{2} z^{2}\left(\frac{q_{i} q_{j}}{q^{2}}+\delta_{i z} \delta_{j z}\right)\right] .
\end{gathered}
$$

Using the fact that all three of the correlation functions $C_{\rho \rho}(\mathbf{q}), C_{p \rho}(\mathbf{q})$, and $C_{p p}(\mathbf{q})$ are proportional to $\frac{1}{q}$ times functions that depend only on the direction $\theta_{\mathbf{q}}$ of $\mathbf{q}$, and making the simple change of variables $\mathbf{q}=\frac{\mathbf{Q}}{z}$, shows that all three of these (tensor) integrals $\Pi^{(1,2,3)}(z)$ are proportional to $\frac{1}{z}$.

Thus, we conclude that

$$
\left\langle v_{i}\left(\mathbf{r}_{\|}, z\right) v_{j}\left(\mathbf{r}_{\|}, z\right)\right\rangle=\left(\frac{1}{z}\right) \mathbf{M}
$$

where we have defined the constant, parameter dependent matrix

$$
\mathbf{M} \equiv v_{0}^{2} \mathbf{M}_{i j}^{(1)}+v_{0} v_{a}^{\prime} \mathbf{M}_{i j}^{(2)}+v_{a}^{\prime 2} \mathbf{M}_{i j}^{(3)}
$$

with

$$
\begin{gathered}
\mathbf{M}_{i j}^{(1)}(z) \equiv \int \frac{d^{2} Q}{(2 \pi)^{2}} \frac{f_{p p}\left(\theta_{\mathbf{Q}}\right)}{Q} e^{-2 Q}\left[\delta_{i y} \delta_{j y}\left(1-\frac{2 Q_{y}^{2}}{Q}\right)+Q_{y}^{2}\left(\frac{Q_{i} Q_{j}}{Q^{2}}+\delta_{i z} \delta_{j z}\right)\right], \\
\mathbf{M}_{i j}^{(2)}(z) \equiv \int \frac{d^{2} Q}{(2 \pi)^{2}} \frac{f_{p \rho}\left(\theta_{\mathbf{Q}}\right)}{Q} e^{-2 Q}\left[1-Q+\frac{2 Q_{x}^{2} Q_{y}^{2}}{Q^{2}}\right]\left(\delta_{i y} \delta_{j x}+\delta_{i x} \delta_{j y}\right) \\
\mathbf{M}_{i j}^{(3)}(z) \equiv \int \frac{d^{2} Q}{(2 \pi)^{2}} \frac{f_{\rho \rho}\left(\theta_{\mathbf{Q}}\right)}{Q} e^{-2 Q}\left[\delta_{i x} \delta_{j x}\left(1-\frac{2 Q_{x}^{2}}{Q}\right)+Q_{x}^{2}\left(\frac{Q_{i} Q_{j}}{Q^{2}}+\delta_{i z} \delta_{j z}\right)\right] .
\end{gathered}
$$

In light of the above discussion, $\mathbf{M}$ is a symmetric matrix, $\quad$ whose only non-zero off-diagonal components are $M_{x y}=$ 
$M_{y x}$. Its diagonal entries are all, in general, different from each other, and from $M_{x y}$.

The important point about these velocity correlations is that they scale like $\frac{1}{z}$; that is, inversely proportional to the distance $z$ from the solid surface. This $\frac{1}{z}$ scaling will break down once $z$ becomes microscopic, as can be seen as follows: our arguments above depended on our hydrodynamic theory, which breaks down for wavevectors $q$ comparable to an inverse microscopic length. Since the integrals over wavevector that we have done to derive VII.7 were dominated by $q \sim \frac{1}{z}$, the calculation clearly ceases to be valid once $z$ is a microscopic length, because then we'll need the correlation functions at wavevectors comparable to an inverse microscopic length, at which our hydrodynamic theory does not apply.

We now calculate the space and time-dependent velocity correlators

$$
C_{i j}^{v}\left(\mathbf{r}_{\|}-\mathbf{r}_{\|}^{\prime}, z, z^{\prime}, t-t^{\prime}\right) \equiv\left\langle v_{i}\left(\mathbf{r}_{\|}, z, t\right) v_{j}\left(\mathbf{r}_{\|}^{\prime}, z^{\prime}, t^{\prime}\right)\right\rangle
$$

This can also be written in terms of the in terms of the polarization $\hat{\mathbf{p}}$ and density $\rho$ correlation functions using the relation A21 between the bulk fluid velocity field and the surface velocity, and our boundary condition (III.14 for that surface velocity. We find

$$
\begin{aligned}
& C_{i j}^{v}\left(\mathbf{r}_{\|}-\mathbf{r}_{\|}^{\prime}, z, z^{\prime}, t-t^{\prime}\right)=\int \frac{d \omega}{2 \pi} \frac{d^{2} q}{(2 \pi)^{2}} \exp \left[i \omega\left(t-t^{\prime}\right)-i \mathbf{q} \cdot\left(\mathbf{r}_{\|}-\mathbf{r}_{\|}^{\prime}\right)\right]\left\langle v_{i}(\mathbf{q}, z, \omega) v_{j}\left(-\mathbf{q}, z^{\prime},-\omega\right)\right\rangle \\
& =v_{0}^{2} \int \frac{d \omega}{2 \pi} \frac{d^{2} q}{(2 \pi)^{2}} \exp \left[i \omega\left(t-t^{\prime}\right)-i \mathbf{q} \cdot\left(\mathbf{r}_{\|}-\mathbf{r}_{\|}^{\prime}\right)-q\left(z+z^{\prime}\right)\right] C_{p p}(\mathbf{q}, \omega)\left[\delta_{i y} \delta_{j y}\left(1-\frac{q_{y}^{2}\left(z+z^{\prime}\right)}{q}\right)+q_{y}^{2} z z^{\prime}\left(\frac{q_{i} q_{j}}{q^{2}}+\delta_{i z} \delta_{j z^{\prime}}\right)\right] \\
& +v_{0} v_{a}^{\prime} \int \frac{d \omega}{2 \pi} \frac{d^{2} q}{(2 \pi)^{2}} \exp \left[i \omega\left(t-t^{\prime}\right)-i \mathbf{q} \cdot\left(\mathbf{r}_{\|}-\mathbf{r}_{\|}^{\prime}\right)-q\left(z+z^{\prime}\right)\right] C_{p \rho}(\mathbf{q}, \omega)\left[\left(\delta_{i x} \delta_{j y}+\delta_{i y} \delta_{j x}\right)\left(1-\frac{q\left(z+z^{\prime}\right)}{2}+\frac{2 z z^{\prime} q_{x}^{2} q_{y}^{2}}{q^{2}}\right)\right] \\
& +v_{a}^{\prime 2} \int \frac{d \omega}{2 \pi} \frac{d^{2} q}{(2 \pi)^{2}} \exp \left[i \omega\left(t-t^{\prime}\right)-i \mathbf{q} \cdot\left(\mathbf{r}_{\|}-\mathbf{r}_{\|}^{\prime}\right)-q\left(z+z^{\prime}\right)\right] C_{\rho \rho}(\mathbf{q}, \omega)\left[\delta_{i x} \delta_{j x}\left(1-\frac{q_{x}^{2}\left(z+z^{\prime}\right)}{q}\right)+q_{x}^{2} z z^{\prime}\left(\frac{q_{i} q_{j}}{q^{2}}+\delta_{i z} \delta_{j z^{\prime}}\right)\right]
\end{aligned}
$$

where $C_{p p}(\mathbf{q}, \omega), C_{p \rho}(\mathbf{q}, \omega)$, and $C_{\rho \rho}(\mathbf{q}, \omega)$ are given by equations (II.13) and (V.8), (II.14), (V.10), (II.15), and (V.12), of the introduction.

One of the best experimental probes of this correlation function is the diffusion of tracer particles. Consider first neutrally buoyant tracer particles, which, in the absence of diffusion, sit at a constant $z$, and are advected along $\hat{\mathbf{x}}$ at a speed $v_{0}$ by the mean motion of the fluid. If we wish to study the diffusion of these particles on a time scale small compared to the time required for them to diffuse a distance comparable to $z$, we therefore only need the velocity correlations (VII.13) at $\mathbf{r}-\mathbf{r}^{\prime}=v_{0}\left(t-t^{\prime}\right) \hat{\mathbf{x}}$, $z=z^{\prime}$. In this limit, (VII.13) reduces to

$C_{i j}^{v}\left(v_{0}\left(t-t^{\prime}\right) \hat{\mathbf{x}}, z, z, t-t^{\prime}\right)=C_{i j}^{p p}\left(v_{0}\left(t-t^{\prime}\right) \hat{\mathbf{x}}, z, z, t-t^{\prime}\right)+C_{i j}^{p \rho}\left(v_{0}\left(t-t^{\prime}\right) \hat{\mathbf{x}}, z, z, t-t^{\prime}\right)+C_{i j}^{\rho \rho}\left(v_{0}\left(t-t^{\prime}\right) \hat{\mathbf{x}}, z, z, t-t^{\prime}\right)$ 


$$
\begin{aligned}
& C_{i j}^{p p}\left(v_{0}\left(t-t^{\prime}\right) \hat{\mathbf{x}}, z, z, t-t^{\prime}\right)= \\
& v_{0}^{2} \int \frac{d \omega}{2 \pi} \frac{d^{2} q}{(2 \pi)^{2}} \exp \left[i\left(\omega-v_{0} q_{x}\right)\left(t-t^{\prime}\right)-2 q z\right] C_{p p}(\mathbf{q}, \omega)\left[\delta_{i y} \delta_{j y}\left(1-\frac{2 q_{y}^{2} z}{q}\right)+q_{y}^{2} z^{2}\left(\frac{q_{i} q_{j}}{q^{2}}+\delta_{i z} \delta_{j z}\right)\right] \\
& C_{i j}^{p \rho}\left(v_{0}\left(t-t^{\prime}\right) \hat{\mathbf{x}}, z, z, t-t^{\prime}\right)= \\
& v_{a}^{\prime} v_{0} \int \frac{d \omega}{2 \pi} \frac{d^{2} q}{(2 \pi)^{2}} \exp \left[i\left(\omega-v_{0} q_{x}\right)\left(t-t^{\prime}\right)-2 q z\right] C_{p \rho}(\mathbf{q}, \omega)\left[\left(\delta_{i x} \delta_{j y}+\delta_{i y} \delta_{j x}\right)\left(1-q z+\frac{2 z^{2} q_{x}^{2} q_{y}^{2}}{q^{2}}\right)\right] \\
& C_{i j}^{\rho \rho}\left(v_{0}\left(t-t^{\prime}\right) \hat{\mathbf{x}}, z, z, t-t^{\prime}\right)= \\
& v_{a}^{\prime 2} \int \frac{d \omega}{2 \pi} \frac{d^{2} q}{(2 \pi)^{2}} \exp \left[i\left(\omega-v_{0} q_{x}\right)\left(t-t^{\prime}\right)-2 q z\right] C_{\rho \rho}(\mathbf{q}, \omega)\left[\delta_{i x} \delta_{j x}\left(1-\frac{2 q_{x}^{2} z}{q}\right)+q_{x}^{2} z^{2}\left(\frac{q_{i} q_{j}}{q^{2}}+\delta_{i z} \delta_{j z}\right)\right]
\end{aligned}
$$

It is straightforward to see from this expression that the tensor $C_{i j}^{p p}\left(v_{0}\left(t-t^{\prime}\right) \hat{\mathbf{x}}, z, z, t-t^{\prime}\right)$ is purely diagonal. Begin by noting that the only term in the integrand in VII.15 that has an off-diagonal component is the $q_{i} q_{j}$ term. Since $\mathbf{q}$ is a vector in the $x y$ plane, this term vanishes if either index $i$ or $j$ is $z$. Hence, the only offdiagonal terms are $i=x, j=y$, or vis-versa. In either case, the integrand then becomes odd in $q_{y}$ (recall that both $C_{p p}(\mathbf{q}, \omega)$ and $q=|\mathbf{q}|$ are even in $\mathbf{q}$. These are the only $q_{y}$ dependent pieces of the rest of the integrand (it appears in the $2 q z$ term in the argument of the first exponential)).

Hence, the integral in (VII.15), and, therefore, the correlation function $C_{i j}^{p p}\left(v_{0}\left(t-t^{\prime}\right) \hat{\mathbf{x}}, z, z, t-t^{\prime}\right)$, itself vanishes if $i=x, j=y$, or vice-versa. Since we have already established that the off-diagonal components of $C_{i j}^{p p}\left(v_{0}\left(t-t^{\prime}\right) \hat{\mathbf{x}}, z, z, t-t^{\prime}\right)$ with one of the indices equal to $z$ also vanish, this completes the proof that $C_{i j}^{p p}\left(v_{0}\left(t-t^{\prime}\right) \hat{\mathbf{x}}, z, z, t-t^{\prime}\right)$ is, as claimed earlier, purely diagonal. Similarly we can show from VII.15 that $C_{i j}^{\rho \rho}\left(v_{0}\left(t-t^{\prime}\right) \hat{\mathbf{x}}, z, z, t-t^{\prime}\right)$ is also a diagonal tensor.

However $C_{i j}^{p \rho}\left(v_{0}\left(t-t^{\prime}\right) \hat{\mathbf{x}}, z, z, t-t^{\prime}\right)$ is an off-diagonal symmetric tensor, as is easily seen from VII.15). Only the components $i=x, j=y$, and $i=y, j=x$ are non-zero, and equal.

This shows that $C_{i j}^{v}\left(v_{0}\left(t-t^{\prime}\right) \hat{\mathbf{x}}, z, z, t-t^{\prime}\right)$ as a whole is symmetric tensor with four independent components. The long-time scaling behavior of the four non-zero independent components $C_{x x}^{v}\left(v_{0}\left(t-t^{\prime}\right) \hat{\mathbf{x}}, z, z, t-t^{\prime}\right)$, $C_{y y}^{v}\left(v_{0}\left(t-t^{\prime}\right) \hat{\mathbf{x}}, z, z, t-t^{\prime}\right)$ and $C_{x y}^{v}\left(v_{0}\left(t-t^{\prime}\right) \hat{\mathbf{x}}, z, z, t-\right.$ $\left.t^{\prime}\right)$ are essentially same, as shown below. However, $C_{z z}^{v}\left(v_{0}\left(t-t^{\prime}\right) \hat{\mathbf{x}}, z, z, t-t^{\prime}\right)$ has a different behavior in the long time limit. This is significant: diffusion along any direction in the $x y$-plane is anomalous, whereas it is normal (non-anomalous) along the vertical or $z$-direction.

To see this, let us consider each of these four non-zero components in turn, starting with $C_{x x}^{v}\left(v_{0}\left(t-t^{\prime}\right) \hat{\mathbf{x}}, z, z, t-\right.$ $\left.t^{\prime}\right)$. Using (VII.14), we see that this can be expressed as

$$
C_{x x}^{v}\left(v_{0}\left(t-t^{\prime}\right) \hat{\mathbf{x}}, z, z, t-t^{\prime}\right)=C_{x x}^{p p}\left(v_{0}\left(t-t^{\prime}\right) \hat{\mathbf{x}}, z, z, t-t^{\prime}\right)+C_{x x}^{\rho \rho}\left(v_{0}\left(t-t^{\prime}\right) \hat{\mathbf{x}}, z, z, t-t^{\prime}\right)
$$

as $C_{x x}^{p \rho}\left(v_{0}\left(t-t^{\prime}\right) \hat{\mathbf{x}}, z, z, t-t^{\prime}\right)$ is zero, being a non-diagonal tensor itself.
We evaluate each of the two terms in (VII.16). From (VII.15), we see that $C_{x x}^{p p}\left(v_{0}\left(t-t^{\prime}\right) \hat{\mathbf{x}}, z, z, t-t^{\prime}\right)$ is given by

$$
C_{x x}^{p p}\left(v_{0}\left(t-t^{\prime}\right) \hat{\mathbf{x}}, z, z, t-t^{\prime}\right)=v_{0}^{2} \int \frac{d \omega}{2 \pi} \frac{d^{2} q}{(2 \pi)^{2}} \exp \left[i\left(\omega-v_{0} q_{x}\right)\left(t-t^{\prime}\right)-2 q z\right] C_{p p}(\mathbf{q}, \omega) \frac{q_{x}^{2} q_{y}^{2} z^{2}}{q^{2}}
$$

Making the change of variables of integration from $(\mathbf{q}, \omega)$ to dimensionless variables $\mathbf{Q}, \Omega$ given by

$$
\omega \equiv \frac{v_{0} \Omega}{z} \quad, \quad \mathbf{q} \equiv \frac{\mathbf{Q}}{z},
$$

and recalling that $C_{p p}(\mathbf{q}, \omega)$ obeys the scaling form (II.13, we find that 


$$
C_{x x}^{p p}\left(v_{0}\left(t-t^{\prime}\right) \hat{\mathbf{x}}, z, z, t-t^{\prime}\right)=\left(\frac{v_{0}^{3}}{z}\right) \int \frac{d \Omega}{2 \pi} \frac{d^{2} Q}{(2 \pi)^{2}} \exp \left[i\left(\Omega-Q_{x}\right) v_{0}\left(t-t^{\prime}\right) / z-2 Q\right] F_{p p}\left(\frac{v_{0} \Omega}{Q}, \theta_{\mathbf{Q}}\right) \frac{Q_{x}^{2} Q_{y}^{2}}{Q^{4}}
$$

where $\theta_{\mathbf{Q}}$ is the angle between the rescaled, dimensionless vector $\mathbf{Q}$ and the direction of mean polarization $\hat{\mathbf{x}}$ (which is, of course, just the same as the angle $\theta_{\mathbf{q}}$ between the original vector $\mathbf{q}$ and the direction of mean polarization $\hat{\mathbf{x}}$, since our rescaling (VII.18) was isotropic.

Using our expression $(\mathrm{V} .8)$ for $F_{p p}$, we have

$$
F_{p p}\left(\frac{v_{0} \Omega}{Q}, \theta_{\mathbf{Q}}\right)=\frac{2 D_{p}\left(v_{0} \frac{\Omega}{Q}-v_{\rho} \cos \theta_{\mathbf{Q}}\right)^{2}}{\left(v_{0} \frac{\Omega}{Q}-c_{+}\left(\theta_{\mathbf{Q}}\right)\right)^{2}\left(v_{0} \frac{\Omega}{Q}-c_{-}\left(\theta_{\mathbf{Q}}\right)\right)^{2}+\left(v_{0} \frac{\Omega}{Q} \psi\left(1, \theta_{\mathbf{Q}}\right)-v_{\rho} \psi\left(\varphi, \theta_{\mathbf{Q}}\right) \cos \theta_{\mathbf{Q}}\right)^{2}}
$$

Factoring out $D_{p} v_{0}^{2}$ from the numerator of this expression, and $v_{0}^{4}$ from the denominator, we see that this can be rewritten in terms of a completely dimensionless scal- ing function of dimensionless arguments as

$$
F_{p p}\left(\frac{v_{0} \Omega}{Q}, \theta_{\mathbf{Q}}\right)=\left(\frac{D_{p}}{v_{0}^{2}}\right) H_{p p}\left(\frac{\Omega}{Q}, \theta_{\mathbf{Q}} ;\left\{\frac{v_{\rho}}{v_{0}}, \frac{v_{p}}{v_{0}}, \frac{\gamma}{v_{0}}, \frac{c_{0}}{v_{0}}\right\}\right),
$$

where we have defined

$$
H_{p p}\left(\frac{\Omega}{Q}, \theta_{\mathbf{Q}} ;\left\{\frac{v_{\rho}}{v_{0}}, \frac{v_{p}}{v_{0}}, \frac{\gamma}{v_{0}}, \frac{c_{0}}{v_{0}}\right\}\right)=\frac{2\left(\frac{\Omega}{Q}-\frac{v_{\rho}}{v_{0}} \cos \theta_{\mathbf{Q}}\right)^{2}}{\left(\frac{\Omega}{Q}-\frac{c_{+}\left(\theta_{\mathbf{Q}}\right)}{v_{0}}\right)^{2}\left(\frac{\Omega}{Q}-\frac{c_{-}\left(\theta_{\mathbf{Q}}\right)}{v_{0}}\right)^{2}+\left(\frac{\Omega}{Q} \frac{\psi\left(1, \theta_{\mathbf{Q}}\right)}{v_{0}}-\frac{v_{\rho}}{v_{0}} \frac{\psi\left(\varphi, \theta_{\mathbf{Q}}\right)}{v_{0}} \cos \theta_{\mathbf{Q}}\right)^{2}} .
$$

Some of the dependence of this function on the dimensionless ratios $\frac{v_{\rho}}{v_{0}}, \frac{v_{p}}{v_{0}}, \frac{\gamma}{v_{0}}$, and $\frac{c_{0}}{v_{0}}$ is hidden in the ratios $\frac{c_{ \pm}\left(\theta_{\mathbf{Q}}\right)}{v_{0}}$, since $c_{ \pm}\left(\theta_{\mathbf{Q}}\right)$ depend on $v_{\rho}, v_{p}, \gamma$, and $c_{0}$, as displayed in equation (V.4).

Using (VII.21) and (VII.22) in (VII.19), we find that $C_{x x}^{p p}\left(v_{0}\left(t-t^{\prime}\right) \hat{\mathbf{x}}, z, z, t-t^{\prime}\right)$ itself obeys a simple scaling law:

$$
C_{x x}^{p p}\left(v_{0}\left(t-t^{\prime}\right) \hat{\mathbf{x}}, z, z, t-t^{\prime}\right)=\left(\frac{D_{p} v_{0}}{z}\right) F_{x x}^{p p}\left(\frac{v_{0}\left|t-t^{\prime}\right|}{z}\right)
$$

where the dimensionless scaling function

$$
F_{x x}^{p p}\left(u_{r}\right)=\int \frac{d \Omega}{2 \pi} \frac{d^{2} Q}{(2 \pi)^{2}} \exp \left[i\left(\Omega-Q_{x}\right) u-2 Q\right] H_{p p}\left(\frac{\Omega}{Q}, \theta_{\mathbf{Q}} ;\left\{\frac{v_{\rho}}{v_{0}}, \frac{v_{p}}{v_{0}}, \frac{\gamma}{v_{0}}, \frac{c_{0}}{v_{0}}\right\}\right) \frac{Q_{x}^{2} Q_{y}^{2}}{Q^{4}}
$$

where the dimensionless scaling variable $u_{r} \equiv v_{0}\left|t-t^{\prime}\right| / z$.

The limiting behaviors of $F_{x x}^{p p}\left(u_{r}\right)$ for small and large $u_{r}$ are easy to obtain; and useful: $u_{r} \ll 1$ and $u_{r} \gg 1$ give information about the velocity correlations, and from those, the displacement correlations, in the ballistic and diffusive limits, respectively, as we'll soon show. Intermediate values of $u_{r} \sim \mathcal{O}(1)$ correspond to the crossover between the two regimes. For $u \ll 1$, which corresponds to time differences obeying $v_{0}\left|t-t^{\prime}\right| \ll z$, the $e^{-2 Q}$ factor in the integral VII.24 kills the integrand before (that is, at smaller $Q$ than) the $\left(\Omega-Q_{x}\right) u$ term becomes important. Hence, the integral, and $F_{x x}^{p p}\left(u_{r}\right)$ itself, become independent of $u$ in this limit; that is,

$$
F_{x x}^{p p}\left(u_{r}\right)=A_{x} \text { for } u_{r} \ll 1
$$

where the constant $A_{x x}^{p p}$ is given by

$$
A_{x x}^{p p}=\int \frac{d \Omega}{2 \pi} \frac{d^{2} Q}{(2 \pi)^{2}} e^{-2 Q} H_{p p}\left(\frac{\Omega}{Q}, \theta_{\mathbf{Q}} ;\left\{\frac{v_{\rho}}{v_{0}}, \frac{v_{p}}{v_{0}}, \frac{\gamma}{v_{0}}, \frac{c_{0}}{v_{0}}\right\}\right) \frac{Q_{x}^{2} Q_{y}^{2}}{Q^{4}}
$$


and is a function of all of the ratios $\frac{v_{\rho}}{v_{0}}, \frac{v_{p}}{v_{0}}, \frac{\gamma}{v_{0}}$, and $\frac{c_{0}}{v_{0}}$. $A_{x x}^{p p}$ will be of $\mathcal{O}(1)$ when all of these ratios are of $\mathcal{O}(1)$.

In the opposite limit $u_{r} \gg 1$, which corresponds to time differences obeying $v_{0}\left|t-t^{\prime}\right| \gg z$, the $e^{i\left(\Omega-Q_{x}\right) u}$ factor in the integral VII.24 kills (by oscillation) all contributions to the integral coming from $Q \gtrsim \frac{1}{u_{r}} \ll 1$. Hence, in the dominant region of the integral, $Q \ll 1$, and so we can drop the $2 Q$ term in the argument of the exponential. Doing so gives

$$
F_{x x}^{p p}\left(u_{r}\right)=\int \frac{d \Omega}{2 \pi} \frac{d^{2} Q}{(2 \pi)^{2}} \exp \left[i\left(\Omega-Q_{x}\right) u\right] H_{p p}\left(\frac{\Omega}{Q}, \theta_{\mathbf{Q}} ;\left\{\frac{v_{\rho}}{v_{0}}, \frac{v_{p}}{v_{0}}, \frac{\gamma}{v_{0}}, \frac{c_{0}}{v_{0}}\right\}\right) \frac{Q_{x}^{2} Q_{y}^{2}}{Q^{4}}
$$

This integral can easily be done with one further change of variables:

$$
\Omega \equiv \frac{\Omega^{\prime}}{u_{r}} \quad, \quad \mathbf{Q} \equiv \frac{\mathbf{Q}^{\prime}}{u_{r}},
$$

which gives

$$
F_{x x}^{p p}\left(u_{r}\right)=\frac{B_{x x}^{p p}}{u_{r}^{3}} \text { for } u_{r} \gg 1
$$

where the constant $B_{x x}^{p p}$ is given by

$$
B_{x x}^{p p}=\int \frac{d \Omega^{\prime}}{2 \pi} \frac{d^{2} Q^{\prime}}{(2 \pi)^{2}} \exp \left[i\left(\Omega^{\prime}-Q_{x}^{\prime}\right)\right] H_{p p}\left(\frac{\Omega^{\prime}}{Q^{\prime}}, \theta_{\mathbf{Q}^{\prime}} ;\left\{\frac{v_{\rho}}{v_{0}}, \frac{v_{p}}{v_{0}}, \frac{\gamma}{v_{0}}, \frac{c_{0}}{v_{0}}\right\}\right) \frac{Q_{x}^{\prime 2} Q_{y}^{\prime 2}}{Q^{\prime}}
$$

and, like $A_{x x}^{p p}$, is again a function of all of the ratios $\frac{v_{\rho}}{v_{0}}$, $\frac{v_{p}}{v_{0}}, \frac{\gamma}{v_{0}}$, and $\frac{c_{0}}{v_{0}}$, and will again be of $O(1)$ when all of these ratios are of $O(1)$.

In summary, the behavior of the scaling function
$F_{x x}^{p p}\left(u_{r}\right)$ is given by:

$$
F_{x x}^{p p}\left(u_{r}\right)=\left\{\begin{array}{lll}
A_{x x}^{p p} & , & u_{r} \ll 1 \\
\frac{B_{x x}^{p p}}{u_{r}^{3}} & , & u_{r} \gg 1
\end{array},\right.
$$

We now evaluate $C_{x x}^{\rho \rho}\left(v_{0}\left(t-t^{\prime}\right) \hat{\mathbf{x}}, z, z, t-t^{\prime}\right)$, which from VII.15) is expressed as

$$
C_{x x}^{\rho \rho}\left(v_{0}\left(t-t^{\prime}\right) \hat{\mathbf{x}}, z, z, t-t^{\prime}\right)=v_{a}^{\prime 2} \int \frac{d \omega}{2 \pi} \frac{d^{2} q}{(2 \pi)^{2}} \exp \left[i\left(\omega-v_{0} q_{x}\right)\left(t-t^{\prime}\right)-2 q z\right] C_{\rho \rho}(\mathbf{q}, \omega)\left[1-\frac{2 q_{x}^{2} z}{q}+\frac{z^{2} q_{x}^{4}}{q^{2}}\right]
$$

Making the same change of variables VII.18 as before, we obtain an scaling form for $C_{i j}^{\rho \rho}\left(v_{0}\left(t-t^{\prime}\right) \hat{\mathbf{x}}, z, z, t-\right.$ $\left.t^{\prime}\right)$, given by

$C_{x x}^{\rho \rho}\left(v_{0}\left(t-t^{\prime}\right) \hat{\mathbf{x}}, z, z, t-t^{\prime}\right)=\left(\frac{D_{p} \rho_{c}^{2} v_{a}^{\prime 2}}{v_{0} z}\right) F_{x x}^{\rho \rho}\left(\frac{v_{0}\left|t-t^{\prime}\right|}{z}\right)$

where $F_{x x}^{\rho \rho}\left(\frac{v_{0}\left|t-t^{\prime}\right|}{z}\right)$ is written as

$$
F_{x x}^{\rho \rho}\left(u_{r}\right)=\int \frac{d \Omega}{2 \pi} \frac{d^{2} Q}{(2 \pi)^{2}} \exp \left[i\left(\Omega-Q_{x}\right) u_{r}-2 Q\right] H_{\rho \rho}\left(\frac{\Omega}{Q}, \theta_{\mathbf{Q}} ;\left\{\frac{v_{\rho}}{v_{0}}, \frac{v_{p}}{v_{0}}, \frac{\gamma}{v_{0}}, \frac{c_{0}}{v_{0}}\right\}\right)\left[\frac{1}{Q^{2}}-\frac{2 Q_{x}^{2}}{Q^{3}}+\frac{Q_{x}^{4}}{Q^{4}}\right]
$$




$$
H_{\rho \rho}\left(\frac{\Omega}{Q}, \theta_{\mathbf{Q}} ;\left\{\frac{v_{\rho}}{v_{0}}, \frac{v_{p}}{v_{0}}, \frac{\gamma}{v_{0}}, \frac{c_{0}}{v_{0}}\right\}\right)=\frac{2\left(\frac{v_{\rho}}{v_{0}}\right)^{2}\left(\sin \theta_{\mathbf{Q}}\right)^{2}}{\left(\frac{\Omega}{Q}-\frac{c_{+}\left(\theta_{\mathbf{Q}}\right)}{v_{0}}\right)^{2}\left(\frac{\Omega}{Q}-\frac{c_{-}\left(\theta_{\mathbf{Q}}\right)}{v_{0}}\right)^{2}+\left(\frac{\Omega}{Q} \frac{\psi\left(1, \theta_{\mathbf{Q}}\right)}{v_{0}}-\frac{v_{\rho}}{v_{0}} \frac{\psi\left(\varphi, \theta_{\mathbf{Q}}\right)}{v_{0}} \cos \theta_{\mathbf{Q}}\right)^{2}}
$$

The limiting behaviors of the scaling function $F_{x x}^{\rho \rho}(u)$ can be obtained by an almost identical analysis to that used for $F_{x x}^{p p}\left(u_{r}\right)$, giving:

$$
F_{x x}^{\rho \rho}\left(u_{r}\right)= \begin{cases}A_{x x}^{\rho \rho}, & u \ll 1 \\ \frac{\left(B_{x x}^{\rho \rho}\right)_{1}}{u_{r}}+\frac{\left(B_{x x}^{\rho \rho}\right)_{2}}{u_{r}^{2}}+\frac{\left(B_{x x}^{\rho \rho}\right)_{3}}{u_{r}^{3}}, & u_{r} \gg 1\end{cases}
$$

with

$$
A_{x x}^{\rho \rho}=\int \frac{d \Omega}{2 \pi} \frac{d^{2} Q}{(2 \pi)^{2}} e^{-2 Q} H_{\rho \rho}\left(\frac{\Omega}{Q}, \theta_{\mathbf{Q}} ;\left\{\frac{v_{\rho}}{v_{0}}, \frac{v_{p}}{v_{0}}, \frac{\gamma}{v_{0}}, \frac{c_{0}}{v_{0}}\right\}\right)\left[\frac{1}{Q^{2}}-\frac{2 Q_{x}^{2}}{Q^{3}}+\frac{Q_{x}^{4}}{Q^{4}}\right]
$$

and

$$
\begin{gathered}
\left(B_{x x}^{\rho \rho}\right)_{1}=\int \frac{d \Omega^{\prime}}{2 \pi} \frac{d^{2} Q^{\prime}}{(2 \pi)^{2}} \exp \left[i\left(\Omega^{\prime}-Q_{x}^{\prime}\right)\right] H_{\rho \rho}\left(\frac{\Omega^{\prime}}{Q^{\prime}}, \theta_{\mathbf{Q}^{\prime}} ;\left\{\frac{v_{\rho}}{v_{0}}, \frac{v_{p}}{v_{0}}, \frac{\gamma}{v_{0}}, \frac{c_{0}}{v_{0}}\right\}\right) \frac{1}{Q^{\prime 2}}, \\
\left(B_{x x}^{\rho \rho}\right)_{2}=-2 \int \frac{d \Omega^{\prime}}{2 \pi} \frac{d^{2} Q^{\prime}}{(2 \pi)^{2}} \exp \left[i\left(\Omega^{\prime}-Q_{x}^{\prime}\right)\right] H_{\rho \rho}\left(\frac{\Omega^{\prime}}{Q^{\prime}}, \theta_{\mathbf{Q}^{\prime}} ;\left\{\frac{v_{\rho}}{v_{0}}, \frac{v_{p}}{v_{0}}, \frac{\gamma}{v_{0}}, \frac{c_{0}}{v_{0}}\right\}\right) \frac{Q_{x}^{\prime 2}}{Q^{\prime 3}}, \\
\left(B_{x x}^{\rho \rho}\right)_{3}=\int \frac{d \Omega^{\prime}}{2 \pi} \frac{d^{2} Q^{\prime}}{(2 \pi)^{2}} \exp \left[i\left(\Omega^{\prime}-Q_{x}^{\prime}\right)\right] H_{\rho \rho}\left(\frac{\Omega^{\prime}}{Q^{\prime}}, \theta_{\mathbf{Q}^{\prime}} ;\left\{\frac{v_{\rho}}{v_{0}}, \frac{v_{p}}{v_{0}}, \frac{\gamma}{v_{0}}, \frac{c_{0}}{v_{0}}\right\}\right) \frac{Q_{x}^{\prime 4}}{Q^{\prime 4}},
\end{gathered}
$$

Like $A_{x x}^{p p}$ VII.26) and $B_{x x}^{p p}$ (VII.28), $A_{x x}^{\rho \rho}$ and $\left(B_{x x}^{\rho \rho}\right)_{1}$, $\left(B_{x x}^{\rho \rho}\right)_{2}$, and $\left(B_{x x}^{\rho \rho}\right)_{3}$ are functions of all of the ratios $\frac{v_{\rho}}{v_{0}}$, $\frac{v_{p}}{v_{0}}, \frac{\gamma}{v_{0}}$, and $\frac{c_{0}}{v_{0}}$, and will all be of $O(1)$ when all of these ratios are of $O(1)$.

Taking (VII.23) and VII.30 together, we find that $C_{x x}^{v}\left(v_{0}\left(t-t^{\prime}\right) \hat{\mathbf{x}}, z, z, t-t^{\prime}\right)$ is given by

$$
C_{x x}^{v}\left(v_{0}\left(t-t^{\prime}\right) \hat{\mathbf{x}}, z, z, t-t^{\prime}\right)=\left(\frac{D_{p} v_{0}}{z}\right) F_{x x}^{p p}\left(\frac{v_{0}\left|t-t^{\prime}\right|}{z}\right)+\left(\frac{D_{p} \rho_{c}^{2} v_{a}^{\prime 2}}{v_{0} z}\right) F_{x x}^{\rho \rho}\left(\frac{v_{0}\left|t-t^{\prime}\right|}{z}\right)
$$

with $F_{x x}^{p p}\left(\frac{v_{0}\left|t-t^{\prime}\right|}{z}\right)$, and $F_{x x}^{\rho \rho}\left(\frac{v_{0}\left|t-t^{\prime}\right|}{z}\right)$ given by VII.28 and VII.32 respectively.

Because of the $\frac{1}{u_{r}}$ piece of the scaling function $F_{x x}^{\rho \rho}\left(u_{r}\right)$, the integral of this correlation function over $u$ for fixed $z$ diverges logarithmically at large $u_{r}$. We will show in section (VIII) below that this implies logarithmic superdif- fusion (equations II.5 and II.6) in the $x$ direction.

The derivation of similar results for the remain components of the velocity correlator tensor is given in Appendix $\mathrm{F}$. 


\section{ANOMALOUS DIFFUSION OF TRACERS}

\section{A. Neutrally buoyant tracers}

A neutrally buoyant tracer is a particle that is simply carried around passively by the flows in the bulk fluid. That is, if the particle is at position $r_{p}(t)$ at time $t$, its instantaneous velocity $\dot{\mathbf{r}}_{p}(t)$ is

$$
\dot{\mathbf{r}}_{p}(t)=\mathbf{v}\left(\mathbf{r}_{p}(t), t\right)
$$

where $\mathbf{v}(\mathbf{r}, t)$ is the velocity field of the passive bulk fluid. Therefore, the instantaneous position of a tracer particle $\mathbf{r}_{p}(t)$ that starts at $\mathbf{r}_{p}(t=0)$ at some later time $t$ is given by

$$
\mathbf{r}_{p}(t)-\mathbf{r}_{p}(0)=\int_{0}^{t} \mathbf{v}\left(\mathbf{r}_{p}\left(t^{\prime}\right), t^{\prime}\right) d t^{\prime}
$$

Since diffusion, and even the superdiffusion than we eventually will find, is a much slower process that ballistic transport, we can take, for a neutrally buoyant particle (i.e., one which has no net speed in the $z$-direction),

$$
\mathbf{r}_{p}\left(t^{\prime}\right)=\left(v_{0} t^{\prime}+x(t=0)\right) \hat{\mathbf{x}}+y(0) \hat{\mathbf{y}}+z \hat{\mathbf{z}}
$$

in VIII.2, where we have taken into account the fact that the passive fluid is, on average, flowing along the $\hat{\mathbf{x}}$ direction at a speed $v_{0}$. We have also simply written $z(t=0)$ as $z$. Doing so gives

$\mathbf{r}_{p}(t)-\mathbf{r}_{p}(0)=\int_{0}^{t} \mathbf{v}\left(\left(v_{0} t^{\prime}+x(t=0)\right) \hat{\mathbf{x}}+y(0) \hat{\mathbf{y}}+z \hat{\mathbf{z}}, t^{\prime}\right) d t^{\prime}$
Autocorrelating the $x, y$, and $z$ components of this equation with themselves gives

$$
\left\langle(x(t)-x(0))^{2}\right\rangle=\int_{0}^{t} d t^{\prime} \int_{0}^{t} d t^{\prime \prime} C_{x x}^{v}\left(t^{\prime}-t^{\prime \prime}\right)
$$

$$
\left\langle(y(t)-y(0))^{2}\right\rangle=\int_{0}^{t} d t^{\prime} \int_{0}^{t} d t^{\prime \prime} C_{y y}^{v}\left(t^{\prime}-t^{\prime \prime}\right),
$$

$$
\begin{gathered}
\langle(x(t)-x(0))(y(t)-y(0))\rangle=\int_{0}^{t} d t^{\prime} \int_{0}^{t} d t^{\prime \prime} C_{x y}^{v}\left(t^{\prime}-t^{\prime \prime}\right)=0 \\
\text { (VIII.7) }
\end{gathered}
$$

where the correlation functions $C_{x x}^{v}\left(t^{\prime}-t^{\prime \prime}\right), C_{y y}^{v}\left(t^{\prime}-t^{\prime \prime}\right)$, $C_{x y}^{v}\left(t^{\prime}-t^{\prime \prime}\right)$, and $C_{z z}^{v}\left(t^{\prime}-t^{\prime \prime}\right)$ are precisely those we calculated in equations (VII.36), (C3), and (C17) respectively of the preceding section. We will now use these expressions to obtain the diffusive motion - that is, the small departure from the mean ballistic motion at velocity $v_{0} \hat{\mathbf{x}}$ of tracers in the $\hat{\mathbf{x}}$ direction - for all three directions.

We begin with the $x$-direction. Using our scaling form (VIII.4) (VII.36) for $C_{x x}^{v}\left(t^{\prime}-t^{\prime \prime}\right)$, we can rewrite this as

$$
\left\langle(x(t)-x(0))^{2}\right\rangle=\left(\frac{D_{p} v_{0}}{z}\right) \int_{0}^{t} d t^{\prime} \int_{0}^{t} d t^{\prime \prime} F_{x x}^{p p}\left(\frac{v_{0}\left|t^{\prime}-t^{\prime \prime}\right|}{z}\right)+\left(\frac{D_{p} \rho_{c}^{2} v_{a}^{\prime 2}}{v_{0} z}\right) \int_{0}^{t} d t^{\prime} \int_{0}^{t} d t^{\prime \prime} F_{x x}^{\rho \rho}\left(\frac{v_{0}\left|t^{\prime}-t^{\prime \prime}\right|}{z}\right)
$$

Now, changing variables of integration in the integrals

$$
\left\langle(x(t)-x(0))^{2}\right\rangle=\left(\frac{D_{p} v_{0}}{z}\right) \int_{0}^{t} d t^{\prime} \int_{-t^{\prime}}^{t-t^{\prime}} d \delta t F_{x x}^{p p}\left(\frac{v_{0}|\delta t|}{z}\right)+\left(\frac{D_{p} \rho_{c}^{2} v_{a}^{\prime 2}}{v_{0} z}\right) \int_{0}^{t} d t^{\prime} \int_{-t^{\prime}}^{t-t^{\prime}} d \delta t F_{x x}^{\rho \rho}\left(\frac{v_{0}|\delta t|}{z}\right)
$$

Now recalling, as shown by equations (VII.27) and VII.32, that $F_{x x}^{p p}\left(u_{r}\right) \sim 1 / u_{r}^{3}$, and $F_{x x}^{\rho \rho}\left(u_{r}\right) \sim 1 / u_{r}$ for $u_{r} \gg 1$ (that is, for $t \gg\left(\frac{z}{v_{0}}\right)$ ), we expect the first integral over $\delta t$ on the right hand side of (VIII.10) to converge as its limits go to $\infty$, and the second integral to diverge. Thus the second integral clearly dominates at long times and (VIII.10) can be approximated by

$$
\left\langle(x(t)-x(0))^{2}\right\rangle \approx\left(\frac{D_{p} \rho_{c}^{2} v_{a}^{\prime 2}}{v_{0} z}\right) \int_{0}^{t} d t^{\prime} \int_{-t^{\prime}}^{t-t^{\prime}} d \delta t F_{x x}^{\rho \rho}\left(\frac{v_{0}|\delta t|}{z}\right)
$$

(VIII.11)

Changing variables of integration from $\delta t$ to $u \equiv \frac{v_{0} \delta t}{z}$, 
we obtain for the integral over $\delta t$ :

$$
\int_{-t^{\prime}}^{t-t^{\prime}} d \delta t F_{x x}^{\rho \rho}\left(\frac{v_{0}|\delta t|}{z}\right)=\left(\frac{z}{v_{0}}\right) \int_{-\frac{v_{0} t^{\prime}}{z}}^{\frac{v_{0}\left(t-t^{\prime}\right)}{z}} d u_{r} F_{x x}^{\rho \rho}\left(u_{r}\right)
$$

Using the fact that $F_{x x}^{\rho \rho}\left(u_{r}\right)$ is an even function of $u_{r}$, we can rewrite this as:

$$
\int_{-t^{\prime}}^{t-t^{\prime}} d \delta t F_{x x}^{\rho \rho}\left(\frac{v_{0}|\delta t|}{z}\right)=\left(\frac{z}{v_{0}}\right)\left\{\int_{0}^{\frac{v_{0} t^{\prime}}{z}} d u_{r} F_{x x}^{\rho \rho}\left(u_{r}\right)+\int_{0}^{\frac{v_{0}\left(t-t^{\prime}\right)}{z}} d u_{r} F_{x x}^{\rho \rho}\left(u_{r}\right)\right\}
$$

It is convenient to break up the first integral in this expression into a part coming from $u_{r}<1$ and another part from $u_{r}>1$ :

$$
\int_{0}^{\frac{v_{0} t^{\prime}}{z}} d u_{r} F_{x x}^{\rho \rho}\left(u_{r}\right)=\int_{0}^{1} d u_{r} F_{x x}^{\rho \rho}\left(u_{r}\right)+\int_{1}^{\frac{v_{0} t^{\prime}}{z}} d u_{r} F_{x x}^{\rho \rho}\left(u_{r}\right)
$$

The integral from 0 to 1 in this expression is clearly $O(1)$, since, as we showed earlier, the integrand is. The second integral can be rewritten:

$$
\begin{aligned}
\int_{1}^{\frac{v_{0} t^{\prime}}{z}} d u_{r} F_{x x}^{\rho \rho}\left(u_{r}\right) & =\left(B_{x x}^{\rho \rho}\right)_{1} \int_{1}^{\frac{v_{0} t^{\prime}}{z}} \frac{d u_{r}}{u_{r}} \\
& +\int_{1}^{\frac{v_{0} t^{\prime}}{z}} d u_{r}\left(F_{x x}^{\rho \rho}\left(u_{r}\right)-\frac{\left(B_{x x}^{\rho \rho}\right)_{1}}{u_{r}}\right) .
\end{aligned}
$$

The second integral on the right hand side of this equa- tion VIII.15 is also $\mathcal{O}(1)$, as can be seen from the fact that the integrand is $\mathcal{O}(1)$, and the expression in parentheses falls off like $\frac{1}{u_{r}^{2}}$ for large $u_{r}$, so the integral converges, even if the upper limit is taken to $\infty$. The first integral in VIII.15) is, of course, elementary (about as elementary as they come, in fact!). We thereby obtain:

$$
\int_{0}^{\frac{v_{0} t^{\prime}}{z}} d u_{r} F_{x x}^{\rho \rho}\left(u_{r}\right)=\left(B_{x x}^{\rho \rho}\right)_{1} \ln \left(\frac{v_{0} t^{\prime}}{z}\right)+\mathcal{O}(1)
$$

An almost identical analysis shows that

$$
\int_{0}^{\frac{v_{0}\left(t-t^{\prime}\right)}{z}} d u_{r} F_{x x}^{\rho \rho}\left(u_{r}\right)=\left(B_{x x}^{\rho \rho}\right)_{1} \ln \left(\frac{v_{0}\left(t-t^{\prime}\right)}{z}\right)+\mathcal{O}(1) .
$$

Inserting (VIII.16) and (VIII.17) into VIII.13) gives

$$
\int_{-t^{\prime}}^{t-t^{\prime}} d \delta t F_{x x}^{\rho \rho}\left(\frac{v_{0}|\delta t|}{z}\right)=\left(\frac{z}{v_{0}}\right)\left\{\left(B_{x x}^{\rho \rho}\right)_{1}\left[\ln \left(\frac{v_{0} t^{\prime}}{z}\right)+\ln \left(\frac{v_{0}\left(t-t^{\prime}\right)}{z}\right)\right]+O(1)\right\}
$$

Inserting this in turn into VIII.11, we obtain:

$$
\left\langle(x(t)-x(0))^{2}\right\rangle=\frac{D_{p} \rho_{c}^{2} v_{a}^{\prime 2}}{v_{0}^{2}} \int_{0}^{t} d t^{\prime}\left\{\left(B_{x x}^{\rho \rho}\right)_{1}\left[\ln \left(\frac{v_{0} t^{\prime}}{z}\right)+\ln \left(\frac{v_{0}\left(t-t^{\prime}\right)}{z}\right)\right]+O(1)\right\} .
$$

All of the integrals in this expression are elementary,

yielding our final superdiffusive expression for the mean squared displacement in the $x$ direction:

$$
\left\langle(x(t)-x(0))^{2}\right\rangle=2\left(B_{x x}^{\rho \rho}\right)_{1} \frac{D_{p} \rho_{c}^{2} v_{a}^{\prime 2}}{v_{0}^{2}} t\left(\ln \left(\frac{v_{0} t}{z}\right)+O(1)\right)
$$

which the alert reader will recognize as equation II.5 of

the introduction. 
We have derived this result assuming that the particle has not moved appreciably in the $z$ direction from its original height $z$. This will be true only for $t \ll \frac{z^{2}}{D_{z}}$, where $D_{z}$ is the ( $z$-independent) diffusion constant we calculated below in Eq. VIII.27). For much longer times, the particle will typically be a distance $z(t) \sim \sqrt{D_{z} t}$ above the surface. Since the logarithm is quite insensitive to the precise position (i.e., to factors of $O(1)$ in this estimate of $z$, it will suffice, to leading logarithmic order, to replace $z$ in VIII.20 with $z(t) \sim \sqrt{D_{z} t}$ in VIII.20). Doing so we obtain

$$
\left\langle(x(t)-x(0))^{2}\right\rangle=\left(B_{x x}^{\rho \rho}\right)_{1} \frac{D_{p} \rho_{c}^{2} v_{a}^{\prime 2}}{v_{0}^{2}} t\left(\ln \left(\frac{v_{0}^{2} t}{D_{z}}\right)+\mathcal{O}(1)\right)
$$

which that same alert reader will recognize as equation (II.6) of the introduction.

For short times $t \ll\left(\frac{z}{v_{0}}\right)$ (which, it should be noted, can actually get arbitrarily long as $z \rightarrow \infty$ ), the arguments of $F_{x x}^{p p}$ and $F_{x x}^{\rho \rho}$ are always much less than 1 throughout the region of integration over $t^{\prime}$ and $t^{\prime \prime}$ in (VIII.9). Therefore, $F_{x x}^{p p}$ and $F_{x x}^{\rho \rho}$ can be replaced in that integral by their small $u_{r}$ limits $A_{x x}^{p p}$ and $A_{x x}^{\rho \rho}$ respectively. And because they are constants, we obtain ballistic scaling in this regime:

$$
\left\langle(x(t)-x(0))^{2}\right\rangle=\left[A_{x x}^{p p}\left(\frac{D_{p} v_{0}}{z}\right)+A_{x x}^{\rho \rho}\left(\frac{D_{p} \rho_{c}^{2} v_{a}^{\prime 2}}{v_{0} z}\right)\right] t^{2} \quad, \quad t \ll\left(\frac{z}{v_{0}}\right) .
$$

The interested reader can easily verify that this ballistic behavior matches smoothly onto the $z$-independent diffusive behavior VIII.12 for $t \sim\left(\frac{z}{v_{0}}\right)$, up to logarithmic factors, as it should.

Virtually identical reasoning applies to diffusion in the $y$ direction, with the result:

$$
\left\langle(y(t)-y(0))^{2}\right\rangle=2\left(B_{y y}^{p p}\right)_{1} D_{p} t\left(\ln \left(\frac{v_{0} t}{z}\right)+O(1)\right)
$$

and at very long times,

$$
\left\langle(y(t)-y(0))^{2}\right\rangle=\left(B_{y y}^{p p}\right)_{1} D_{p} t\left(\ln \left(\frac{v_{0}^{2} t}{D_{z}}\right)+O(1)\right) .
$$

The above equation is easy to recognize as II.7) of the introduction.

ballistic for short times $t \ll\left(\frac{z}{v_{0}}\right)$ :

As for $x$-direction, the motion in the $y$-direction is also

$$
\left\langle(y(t)-y(0))^{2}\right\rangle=\left[A_{y y}^{p p}\left(\frac{D_{p} v_{0}}{z}\right)+A_{y y}^{\rho \rho}\left(\frac{D_{p} \rho_{c}^{2} v_{a}^{\prime 2}}{v_{0} z}\right)\right] t^{2} \quad, \quad t \ll\left(\frac{z}{v_{0}}\right) .
$$

Once again, the interested reader can easily verify that 
independent diffusive behavior VIII.23 for $t \sim\left(\frac{z}{v_{0}}\right)$, as it should.

In the $z$-direction, we see that both $F_{z z}^{p p}\left(u_{r}\right) \sim 1 / u_{r}^{3}$, and $F_{z z}^{\rho \rho}\left(u_{r}\right) \sim 1 / u_{r}^{3}$ for $u \gg 1$ or $t \gg\left(\frac{z}{v_{0}}\right)$. This implies diffusive behavior at long times; i.e.

$$
\left\langle(z(t)-z(0))^{2}\right\rangle=2 D_{z} t
$$

with

$$
D_{z}=D_{p} \int_{0}^{\infty} d u_{r} F_{z z}^{p p}(u)+\frac{D_{p} \rho_{c}^{2} v_{a}^{\prime 2}}{v_{0}^{2}} \int_{0}^{\infty} d u_{r} F_{z z}^{\rho \rho}\left(u_{r}\right)
$$

where $D_{z}$ converges to a finite value.

As for motion in the $x$ and $y$ direction, at short times

$$
t \ll\left(\frac{z}{v_{0}}\right) \text { we obtain ballistic scaling for } z \text { : }
$$

$$
\left\langle(z(t)-z(0))^{2}\right\rangle=\left[A_{z z}^{p p}\left(\frac{D_{p} v_{0}}{z}\right)+A_{z z}^{\rho \rho}\left(\frac{D_{p} \rho_{c}^{2} v_{a}^{\prime 2}}{v_{0} z}\right)\right] t^{2} \quad, \quad t \ll\left(\frac{z}{v_{0}}\right) .
$$

As for $x$, this ballistic behavior for $z$ matches smoothly onto the $z$-independent diffusive behavior (VIII.26) for $t \sim\left(\frac{z}{v_{0}}\right)$, as it should.

We can also calculate the off diagonal correlation

$$
\langle(x(t)-x(0))(y(t)-y(0))\rangle=\int_{0}^{t} d t^{\prime} \int_{0}^{t} d t^{\prime \prime} C_{x y}^{v}\left(t^{\prime}-t^{\prime \prime}\right),
$$

which turns out to be zero as $C_{x y}^{v}\left(t^{\prime}-t^{\prime \prime}\right)$ consists of an integral over q of $C_{p \rho}(\mathbf{q})$ times an even function of $q_{y}$. As we have seen from Eq. () that $C_{p \rho}(\mathbf{q})$ is an odd in $q_{y}$, this integral turns out to be zero. So

$$
\langle(x(t)-x(0))(y(t)-y(0))\rangle=0
$$

The above equation is easy to recognize as (II.8) of the introduction.

\section{B. Sedimenting tracers}

For sedimenting particles, we first note that, since we just showed that diffusion in the $z$ direction is normal, and homogeneous, we need to consider diffusive motion in the $x$, and $y$ direction as well as the $x-y$ plane. We'll start by considering sedimenting particles whose sedimentation speed $v_{\text {sed }}<<v_{0}$. Such particles will spend a time of $O\left(\frac{z_{0}}{v_{\text {sed }}}\right)$ at a height of order $z_{0}$. Due to the aforementioned insensitivity of the logarithmic factor in VIII.20, VIII.23), and the (VIII.30, we can therefore accurately estimate the total mean squared $y$ displacement $\left\langle\left(x\left(z=z_{0}\right)-x(z=0)\right)^{2}\right\rangle,\left\langle\left(y\left(z=z_{0}\right)-y(z=0)\right)^{2}\right\rangle$, $\left\langle\left(x\left(z=z_{0}\right)-x(z=0)\right)\left(y\left(z=z_{0}\right)-y(z=0)\right)\right\rangle$ of a sedimenting particle that starts at $z=z_{0}$ and sinks at speed $v_{\text {sed }}$ all the way down to the surface by simply replacing $t$ in (VIII.20), VIII.23), and VIII.30 equations by $\frac{z_{0}}{v_{\text {sed }}}$, which is the time it takes the sedimenting tracer to sink to the bottom. Doing so gives

$$
\begin{aligned}
\left\langle\left(x\left(z=z_{0}\right)-x(z=0)\right)^{2}\right\rangle & =2\left(B_{x x}^{\rho \rho}\right)_{1} \frac{D_{p} \rho_{c}^{2} v_{a}^{\prime 2}}{v_{0}^{2}}\left(\frac{z_{0}}{v_{\text {sed }}}\right)\left(\ln \left(\frac{v_{0}}{v_{\text {sed }}}\right)+O(1)\right), \\
\left\langle\left(y\left(z=z_{0}\right)-y(z=0)\right)^{2}\right\rangle & =2 B_{y y}^{p p} D_{p}\left(\frac{z_{0}}{v_{\text {sed }}}\right)\left(\ln \left(\frac{v_{0}}{v_{\text {sed }}}\right)+O(1)\right) \\
\left\langle\left(x\left(z=z_{0}\right)-x(z=0)\right)\left(y\left(z=z_{0}\right)-y(z=0)\right)\right\rangle & =0
\end{aligned}
$$

which are just the equations tion.

For more rapidly sedimenting particles (i.e., denser ones), for which $v_{\text {sed }} \gg v_{0}$, the time scale $t \sim \frac{z_{0}}{v_{\text {sed }}}$ of the sinking of the sedimenting particle is in the ballistic regime $t \ll\left(\frac{z}{v_{0}}\right)$, and so we need to use VIII.22 and VIII.25 with $t$ replaced with the sedimenting time 


$$
\begin{aligned}
& \left\langle\left(x\left(z=z_{0}\right)-x(z=0)\right)^{2}\right\rangle=A_{x x}^{p p}\left(\frac{D_{p} v_{0} z_{0}}{v_{\text {sed }}^{2}}\right)+A_{x x}^{\rho \rho}\left(\frac{D_{p} \rho_{c}^{2} v_{a}^{\prime 2} z_{0}}{v_{0} v_{\text {sed }}^{2}}\right), \\
& \left\langle\left(y\left(z=z_{0}\right)-y(z=0)\right)^{2}\right\rangle=A_{y y}^{p p}\left(\frac{D_{p} v_{0} z_{0}}{v_{\mathrm{sed}}^{2}}\right)+A_{y y}^{\rho \rho}\left(\frac{D_{p} \rho_{c}^{2} v_{a}^{\prime 2} z_{0}}{v_{0} v_{\mathrm{sed}}^{2}}\right),
\end{aligned}
$$

These are equations (II.9) and (II.10) of the introduction.

We close this Section by noting that, for both neutrally buoyant particles and sedimenting tracers, the aspect ratio $\sqrt{(y(t)-y(0))^{2} /(x(t)-x(0))^{2}}$ is not exactly 1 , but is a model parameter dependent $\mathcal{O}(1)$ number, reflecting the geometric anisotropy due to the $x$-direction being the preferred direction of orientation for the polar particles. Nonetheless, the aspect ratio is independent of time $t$. This means an anisotropy exponent $\zeta_{a n i}$ that describes the relative scaling between distances measured along the $x$ - and $y$-directions is unity (i.e., $\zeta_{a n i}=1$ ), unlike in the Toner-Tu model of flocking, for which $\zeta_{a n i} \neq 1$ [13, 14] due to strongly relevant anharmonic effects.

The anomalous diffusion we find here has some features common with the phenomenon of "Taylor diffusion" 55, which occurs in parallel plate shear flow. One could obtain the Taylor diffusion geometry from ours by replacing the active fluid layer at the bottom by a flat surface, moving at constant velocity, and our free top surface with a stationary rigid wall with no-slip boundary conditions. However, our anomalous diffusion is fundamentally different from Taylor diffusion. One principal difference is that, in our case, anomalous diffusion arises purely due to the fluctuations away from uniform alignment and motion in the active layer. There is obviously no analog of this if one replaces the active layer in our problem with a uniformly moving rigid body, as in Taylor diffusion.

\section{RENORMALIZATION GROUP ARGUMENT AND IRRELEVANCE OF NON-LINEARITIES}

So far, we have worked strictly with the linear theory. For dry active matter, it is well-known 13 15] that non-linear effects radically change the long-wavelength behavior (indeed, it is only the effects of non-linearities that even make the ordered state in two dimensions pos- sible). It therefore clearly behooves us to ask whether non-linearities have such important effects in our problem.

In this section, we will use a simple renormalization group power counting argument to show that they do not. In fact, the linear theory presented earlier is asymptotically exact at long distances.

There are several sources of nonlinearities. For instance, treating the various speeds and pressures in the problem as functions of $\rho$, and then expanding in powers of $\delta \rho$ produces these nonlinear terms. In addition there are nonlinear terms which originate from the fixed length constant on the polarization $\mathbf{p}$.

In order to ascertain the relevance or irrelevance of the nonlinear effects, we need to consider the lowest order nonlinear terms in the equations for $p_{y}$ and $\rho$, and in the "active partial slip" boundary condition (III.14). The RG analysis we are about to present will make it clear that the most important terms at long distances are those with the fewest possible spatial derivatives, and the smallest number of fields.

There are two types of additional terms. The first type arises very straightforwardly from expanding, e.g, the pressures $P_{p, s}(\rho)$ and the velocity $v_{a}(\rho)$, to higher order in $\rho$. The second come from including the $p_{k} p_{i}$ piece of the transverse projection operator $T_{k i}=\delta_{k i}-p_{k} p_{i}$ in the full equation of motion (III.15). The latter gives rise to the following extra quadratic order in the fields $p_{y}$ and $\delta \rho$ contribution to $\partial_{t} p_{y}$ :

$$
\partial_{t} p_{y}\left(\mathbf{r}_{\|}, t\right)_{\text {quad }}=-\alpha p_{y} v_{s x}\left(\mathbf{r}_{\|}, t\right) .
$$

Since this term already has one power of $p_{y}$ multiplying $v_{s x}$, it is obviously sufficient, to quadratic order in the fields, to use our linear solution (III.46) for $v_{s x}$ in (IX.1). Since it is most convenient for our RG analysis to work in real space, we rewrite (IX.1) in real space, where it reads

$\delta v_{s x}\left(\mathbf{r}_{\|}, t\right)=\left(v_{a}^{\prime}+(\bar{\zeta}-\sigma) \partial_{x}\right) \delta \rho\left(\mathbf{r}_{\|}, t\right)-\mu \eta v_{a}^{\prime} \int d^{2} r_{\perp}^{\prime} \mathcal{K}_{p p \rho}\left(\mathbf{r}_{\|}-\mathbf{r}_{\|}^{\prime}\right) \delta \rho\left(\mathbf{r}_{\|}^{\prime}, t\right)+\zeta_{20} \partial_{y} p_{y}\left(\mathbf{r}_{\|}, t\right)-\mu \eta v_{0} \int d^{2} r_{\perp}^{\prime} \mathcal{K}_{p \rho}\left(\mathbf{r}_{\|}-\mathbf{r}_{\|}^{\prime}\right) p_{y}\left(\mathbf{r}_{\|}^{\prime}, t\right)$

where the kernels $\mathcal{K}_{p p \rho}\left(\mathbf{r}_{\|}\right)$and $\mathcal{K}_{p \rho}\left(\mathbf{r}_{\|}\right)$are the inverse two-dimensional Fourier transforms of $\left(\frac{q^{2}+q_{x}^{2}}{q}\right)$ 
and $\left(\frac{q_{x} q_{y}}{q}\right)$ respectively [50, and are given, at large distances 50, by

$$
\mathcal{K}_{p p \rho}\left(\mathbf{r}_{\|}\right) \approx-\frac{3 x^{2}}{2 \pi r_{\perp}^{5}}
$$

and

$$
\mathcal{K}_{p \rho}\left(\mathbf{r}_{\|}\right) \approx-\frac{3 x y}{2 \pi r_{\perp}^{5}}
$$

as we demonstrate in appendix C. Including such terms, the equations of motion take the form

$$
\begin{aligned}
\frac{\partial p_{y}}{\partial t} & =-v_{p} \partial_{x} p_{y}-\gamma \int d^{2} r_{\perp}^{\prime} \mathcal{K}_{p p}\left(\mathbf{r}_{\|}-\mathbf{r}_{\|}^{\prime}\right) p_{y}\left(\mathbf{r}_{\|}^{\prime}\right)-\frac{\gamma_{\rho}}{\rho_{c}} \int d^{2} r_{\perp}^{\prime} \mathcal{K}_{p \rho}\left(\mathbf{r}_{\|}-\mathbf{r}_{\|}^{\prime}\right) \delta \rho\left(\mathbf{r}_{\|}^{\prime}\right)-\sigma_{t} \partial_{y} \delta \rho+f_{y} \\
& -\gamma_{N L} \int d^{2} r_{\perp}^{\prime} p_{y}\left(\mathbf{r}_{\|}\right) \mathcal{K}_{p \rho}\left(\mathbf{r}_{\|}-\mathbf{r}_{\|}^{\prime}\right) p_{y}\left(\mathbf{r}_{\|}^{\prime}\right)-g_{p p \rho 1} \int d^{2} r_{\perp}^{\prime} p_{y}\left(\mathbf{r}_{\|}\right) \mathcal{K}_{p p \rho}\left(\mathbf{r}_{\|}-\mathbf{r}_{\|}^{\prime}\right) \delta \rho\left(\mathbf{r}_{\|}^{\prime}\right) \\
& -g_{p p \rho 2} \int d^{2} r_{\perp}^{\prime} p_{y}\left(\mathbf{r}_{\|}^{\prime}\right) \mathcal{K}_{p p}\left(\mathbf{r}_{\|}-\mathbf{r}_{\|}^{\prime}\right) \delta \rho\left(\mathbf{r}_{\|}^{\prime}\right)-\sigma_{N L} p_{y} \partial_{x} \delta \rho \\
& +\frac{\lambda_{1}}{2} \partial_{y} p_{y}^{2}+\lambda_{2} \delta \rho \partial_{x} p_{y}+\lambda_{3} p_{y} f_{x}
\end{aligned}
$$

and

$$
\frac{\partial \delta \rho}{\partial t}=-v_{\rho} \partial_{x} \delta \rho-v_{p} \rho_{c} \partial_{y} p_{y}-\lambda_{4} \partial_{y}\left(p_{y} \delta \rho\right)+\lambda_{5} \partial_{x}(\delta \rho)^{2}+\nabla \cdot f_{\rho}
$$

In equation (IX.5), the non-linear terms appear on the last three lines. The "bare" values of the parameters in these equations of motion (hereafter denoted by a superscript " 0 ") are related as follows:

$$
\begin{aligned}
& \gamma_{N L}^{0}=\gamma^{0} \\
& g_{p p \rho 1}^{0}=\gamma_{\rho}^{0} / \rho_{c}^{0}, \\
& g_{p p \rho 2}^{0}=\alpha v_{a}^{\prime} \mu \eta, \\
& \sigma_{N L}^{0}=\sigma_{t}^{0} \\
& \lambda_{1}^{0}=\left(\nu_{1}^{0}-\lambda_{\rho v}^{0}\right) v_{0}-\lambda^{0}, \\
& \lambda_{2}^{0}=\left(\frac{\nu_{1}^{0}+1}{2}\right) v_{a}^{\prime 0}-\lambda_{\rho v}^{0}\left(v_{a}^{\prime}\right)^{0}, \\
& \lambda_{3}^{0}=-1 \\
& \lambda_{4}^{0}=v_{\rho}, \\
& \lambda_{5}^{0}=-\rho_{e}^{\prime 0}\left(v_{a}^{\prime}\right)^{0} .
\end{aligned}
$$

However, none of these parameters will continue to maintain these relations to the other parameters upon renormalization, which is why we have introduced them as independent parameters in the equations of motion (IX.5) and (IX.6).

Here, the kernel $\mathcal{K}_{p p}\left(\mathbf{r}_{\|}\right)$is the inverse two-dimensional Fourier transform of $\left(\frac{q^{2}+q_{y}^{2}}{q}\right)$, and is given, at large dis- tances [50], by

$$
\mathcal{K}_{p p}\left(\mathbf{r}_{\|}\right) \approx-\frac{3 y^{2}}{2 \pi r_{\perp}^{5}},
$$

as we demonstrate in appendix $\mathrm{C}$. Notice that we have restored the number-conserving noise $\mathbf{f}_{\rho}$ in (IX.6). That noise is assumed to be of zero-mean and Gaussiandistributed with variance

$$
\left\langle f_{\rho i}(\mathbf{x}, t) f_{\rho j}\left(\left(\mathbf{x}^{\prime}, t^{\prime}\right)\right\rangle=2 D_{\rho} \delta_{i j} \delta\left(\mathbf{x}-\mathbf{x}^{\prime}\right) \delta\left(t-t^{\prime}\right) .\right.
$$

We will now assess the importance of the non-linear terms in these equations of motion using the dynamical renormalization group (DRG). Readers interested in a more complete and pedagogical discussion of the DRG are referred to [51] for the details of this general approach.

This approach begins by decomposing the Fourier modes of the fields $p_{y}(\mathbf{q}, \omega)$ and $\rho(\mathbf{q}, \omega)$, and the noises $f_{p}$ and $f_{\rho}$ into a rapidly varying parts $p_{y}^{>}(\mathbf{q}, \omega), \rho^{>}(\mathbf{q}, \omega)$, $f_{p}^{>}(\mathbf{q}, \omega)$ and $f_{\rho}^{>}(\mathbf{q}, \omega)$, and slowly varying parts $p_{y}^{<}(\mathbf{q}, \omega)$ and $\left.\rho^{>}<\mathbf{q}, \omega\right) f_{p}^{<}(\mathbf{q}, \omega)$ and $f_{\rho}^{<}(\mathbf{q}, \omega)$. The rapidly varying parts are supported in the momentum shell $\Lambda \mathrm{e}^{-\mathrm{d} \ell}<$ $q<\Lambda$, where $\mathrm{d} \ell$ is an infinitesimal and $\Lambda$ is the ultraviolet cutoff. The slowly varying part is supported in $q<\Lambda \mathrm{e}^{-\mathrm{d} \ell}$.

The DRG procedure then consists of two steps. In step 1 , we eliminate the rapidly varying parts $p_{y}^{>}(\mathbf{q}, \omega)$ and 
$\rho^{>}(\mathbf{q}, \omega)$ from the equations of motion. We do this by solving them iteratively for $p_{y}^{>}(\mathbf{q}, \omega)$ and $\rho^{>}(\mathbf{q}, \omega)$. This solution is a series in the non-linearities which depends on the slow fields $p_{y}^{<}(\mathbf{q}, \omega)$ and $\left.\rho^{<} \mathbf{q}, \omega\right)$. We substitute this solution into the equations of motion for the slow fields, and average over the short wavelength components $f_{y}^{>}(\mathbf{q}, \omega)$ and $\mathbf{f}_{\rho}^{>}(\mathbf{q}, \omega)$ of the noises $f_{y}$ and $\mathbf{f}_{\rho}$, which gives a closed EOM for the slow fields $p_{y}^{<}(\mathbf{q}, \omega)$ and $\left.\rho^{<} \mathbf{q}, \omega\right)$.

Step 2 consists of rescaling space and time as follows: $\mathbf{r}_{\perp}^{\prime}=\mathbf{r}_{\perp} \mathrm{e}^{-\mathrm{d} \ell}, t^{\prime}=t \mathrm{e}^{-z \mathrm{~d} \ell}$, where we will choose the "dynamical exponent" $z$ for our convenience. The rescaling of $\mathbf{r}$ has the effect of restoring the ultraviolet cutoff $\Lambda$ to its original value.
We simultaneously rescale the fields $p_{y}\left(\mathbf{r}_{\perp}, t\right)$ and $\rho\left(\mathbf{r}_{\perp}, t\right)$ according to

$$
p_{y}\left(\mathbf{r}_{\perp}, t\right)=e^{\chi_{p} d \ell} p_{y}\left(\mathbf{x}^{\prime}, t^{\prime}\right), \rho\left(\mathbf{r}_{\perp}, t\right)=e^{\chi_{\rho} d \ell} \rho\left(\mathbf{x}^{\prime}, t^{\prime}\right)
$$

where we will also choose the field rescaling exponents $\chi_{p}$ and $\chi_{\rho}$ for our convenience. We then reorganize the resultant EOM so that it has the same form as our original equations of motion, but with all of the coefficients changed, or, to use the standard jargon, "renormalized". This process is then repeated. The result is a set of differential recursion relations for the various parameters in the equations of motion (IX.5) and (IX.6), which are:

$$
\begin{aligned}
& \frac{d D_{p}}{d \ell}=\left(z-2-2 \chi_{p}\right) D_{p}+\text { nonlinear corrections, } \\
& \frac{d D_{\rho}}{d \ell}=\left(z-4-2 \chi_{\rho}\right) D_{\rho}+\text { nonlinear corrections, } \\
& \frac{d \sigma_{t}}{d \ell}=\left(z-1+\chi_{\rho}-\chi_{p}\right) \sigma_{t}+\text { nonlinear corrections }, \\
& \frac{d \gamma}{d \ell}=(z-1) \gamma+\text { nonlinear corrections }, \\
& \frac{d}{d \ell}\left(\frac{\gamma_{\rho}}{\rho_{c}}\right)=\left(z+\chi_{\rho}-\chi_{p}-1\right)\left(\frac{\gamma_{\rho}}{\rho_{c}}\right)+\text { nonlinear corrections, } \\
& \frac{d v_{p}}{d \ell}=(z-1) v_{p}+\text { nonlinear corrections, } \\
& \frac{d v_{\rho}}{d \ell}=(z-1) v_{\rho}+\text { nonlinear corrections }, \\
& \frac{d\left(v_{p} \rho_{c}\right)}{d \ell}=\left(\chi_{p}-\chi_{\rho}+z-1\right) v_{p} \rho_{c}+\text { nonlinear corrections, } \\
& \frac{d \gamma_{N L}}{d \ell}=\left(z+\chi_{p}-1\right) \gamma_{N L}+\text { nonlinear corrections }, \\
& \frac{d g_{p p \rho 1}}{d \ell}=\left(z+\chi_{\rho}-1\right) g_{p p \rho 1}+\text { nonlinear corrections, } \\
& \frac{d g_{p p \rho 2}}{d \ell}=\left(z+\chi_{\rho}-1\right) g_{p p \rho 2}+\text { nonlinear corrections, } \\
& \frac{d \sigma_{N L}}{d \ell}=\left(z+\chi_{\rho}-1\right) \sigma_{N L}+\text { nonlinear corrections }, \\
& \frac{d \lambda_{1}}{d \ell}=\left(z+\chi_{p}-1\right) \lambda_{1}+\text { nonlinear corrections, }
\end{aligned}
$$

$$
\begin{aligned}
\frac{d \lambda_{2}}{d \ell} & =\left(z+\chi_{\rho}-1\right) \lambda_{2}+\text { nonlinear corrections } \\
\frac{d \lambda_{3}}{d \ell} & =\left(\chi_{p}\right) \lambda_{3}+\text { nonlinear corrections } \\
\frac{d \lambda_{4}}{d \ell} & =\left(z+\chi_{p}-1\right) \lambda_{4}+\text { nonlinear corrections } \\
\frac{d \lambda_{5}}{d \ell} & =\left(z+\chi_{\rho}-1\right) \lambda_{4}+\text { nonlinear corrections }
\end{aligned}
$$


and fields. The "non-linear corrections" denote corrections arising due to the couplings between the "fast" and "slow" modes that arise in the equations of motion IX.5 and (IX.6) as a result of the non-linear terms in those equations. Since they arise from those non-linear terms, these terms must vanish when those non-linear terms do, and must, by continuity, be small when the coefficients $\lambda_{i}$ of those non-linear terms are small.

Of course, how small the $\lambda_{i}$ have to be to ensure that the non-linear corrections in the above recursion relations are negligible depends on the linear parameters in the equations of motion, since those determine the size of the fluctuations in the fields. This suggests a very simple, and very standard, way to decide if the non-linear terms are important at long distances and times: simply choose the rescaling exponents $z$ and $\chi_{p, \rho}$ to keep the linear parameters that control the fluctuations fixed. If, with this choice of $z$ and $\chi_{p, \rho}$, the $\lambda_{i}$ all then flow to zero as $\ell \rightarrow \infty$, then the non-linear terms are guaranteed to be unimportant at long distances. This is the approach we will now take.

We begin by noting that the linear parameters which control the size of the fluctuations in the linear theory are $D_{p}, v_{p, \rho}, \sigma_{t}, \gamma, \frac{\gamma_{\rho}}{\rho_{c}}$, and $v_{p} \rho_{c}$, as we showed in section $(\mathrm{V})$ above, where we calculated the fluctuations in the linear theory.

If we assume that all of the $\lambda_{i}$ are initially small enough that the non-linear corrections in the recursion relations (IX.19)-(IX.31) are negligible, then we can keep the above parameters fixed by choosing

$$
\chi_{p}=\chi_{\rho}=-\frac{1}{2}, \quad z=1 .
$$

The choice $z=1$ implies that the scaling argument of the scaling functions $F_{p p}, F_{\rho \rho}$ and $F_{p \rho}$ should be $\omega / q$ (since for general $z$, it would be $\left.\omega / q^{z}\right)$.

Using these choices (IX.36) in the recursion relation for $D_{\rho}$, and again neglecting the non-linear terms, we see that

$$
\frac{d D_{\rho}}{d \ell}=-2 D_{\rho},
$$

which clearly shows that $D_{\rho}(\ell \rightarrow \infty) \rightarrow 0$. Thus, $D_{\rho}$ is irrelevant at long distances and times, which justifies our neglect of it in our earlier, linear analysis.

We also find that all $\lambda_{i}, i=1 \rightarrow 5$ have same recursion relation:

$$
\frac{d \lambda_{i}}{d \ell}=-\left(\frac{1}{2}\right) \lambda_{i}+\text { nonlinear corrections }
$$

and that the other three non-linear coefficients $\gamma_{N L}, g_{p p \rho}$, and $\sigma_{N L}$ have the same RG eigenvalues:

$$
\begin{aligned}
& \frac{d \gamma_{N L}}{d \ell}=-\left(\frac{1}{2}\right) \gamma_{N L}+\text { nonlinear corrections }, \\
& \frac{d g_{p p \rho 1}}{d \ell}=-\left(\frac{1}{2}\right) g_{p p \rho 1}+\text { nonlinear corrections }, \\
& \frac{d g_{p p \rho 2}}{d \ell}=-\left(\frac{1}{2}\right) g_{p p \rho 2}+\text { nonlinear corrections } \\
& \frac{d \sigma_{N L}}{d \ell}=-\left(\frac{1}{2}\right) \sigma_{N L}+\text { nonlinear corrections }
\end{aligned}
$$

Therefore, all of these nonlinearities are also irrelevant at long distances, at least if they are initially small.

Since $\chi_{p, \rho}$ are both $<0$, any terms with more fields are less relevant. Likewise, any fields with more gradients are also less relevant. This includes all possible other nonlinearities. Therefore, all non-linearities are irrelevant. This implies that our linear results are asymptotically exact at long length and time scales.

\section{SUMMARY AND CONCLUSIONS}

In this paper, we have studied the the stability and fluctuations of a large polar-ordered flock at a solid-liquid interface, which is a natural intermediate case between the two previously studied cases of wet and dry polar active fluids. Such a flock is affected by both the friction force from the solid substrate underneath and the long range hydrodynamic interaction mediated by the overlying passive, isotropic bulk fluid. As a result, such a flock is simultaneously momentum nonconserving, but affected by the hydrodynamic interactions of the bulk surrounding fluid. Friction with the substrate also breaks Galilean invariance.

These features lead to novel behavior at long length and time scales, radically different from both dry and wet polar flocks. First of all, a flock of arbitrarily large size with long range polar order is stable for a range of the model parameters. Although this prediction is qualitatively same as that of the original Toner-Tu model for a dry polar-ordered flock [13, 14], there are significant differences. For instance, the effective damping in the present theory is $\mathcal{O}(q)$ in contrast to the $\mathcal{O}\left(q^{2}\right)$ damping in the linearized Toner-Tu model [13, 14] in the long wavelength limit. As a result, fluctuations are significantly smaller at a solid-fluid interface than in the Toner-Tu model. Therefore, the predictions of the linear theory are asymptotically exact in the long wavelength limit. This is contrast to the larger fluctuations in the Toner-Tu model, in which the predictions from the linear theory break down at long length and time scales due to the fluctuations. Furthermore, although both the solid-fluid interface problem treated here and the TonerTu model are anisotropic, we find isotropic scaling in our problem, while the Toner-Tu model exhibits anisotropic scaling [13, 14]. 
We have shown the existence of giant number fluctuation in our model, with the variance of the number scaling as the $3 / 4$ th power of the mean. In addition to this unusual scaling, we also find that the number fluctuations in a given area depend on the shape, as well as the size, of the area.

In addition, we find that the bulk fluid is "stirred" by the active particles on the interface, giving rise to long ranged fluctuations in the bulk fluid velocity. These in turn lead to anomalous diffusion of tagged particles in the bulk fluid. Specifically, we find that the displacement variances $\left\langle(x(t)-x(0))^{2}\right\rangle$ and $\left\langle(y(t)-y(0))^{2}\right\rangle$ in the plane become anomalous, scaling as $t \ln t$ in the large time limit. In contrast, diffusion in the $z$-direction remains normal, i.e., $\left\langle(z(t)-z(0))^{2}\right\rangle$ scales linearly with $t$.

Non-interacting particles sedimenting through the bulk fluid, as a result, will land on the solid substrate in a region whose typical dimensions exhibit an anomalous logarithmic dependence on the sedimenting speed, as summarized in equations (VIII.31)-(VIII.33).

Finally, we have shown that non-linearities are irrelevant to the long-distance, long-time behavior of these systems, in contrast to dry active matter.

There are many possible extensions of the work reported here. One could, for example, consider replacing either the bulk fluid or the bulk solid of our problem with a liquid crystal (e.g., nematic or smectic). One could also ask how the presence of multiple species, instead of one as here, might affect the macroscopic properties.

It would also be interesting to study the order-disorder transition in the present model. Will a linear theory suffice, as we have found it does for the ordered phase?

It would also be interesting to study a variant of our model, in which the passive fluid layer has finite height $h$. This is precisely the experimental geometry of Ref. [28]. In the limit of lateral length scales $L_{\perp} \gg h$, such a system reduces to the system of polar-ordered flocks suspended in a fluid on a substrate studied in Ref. 52. Lastly, one might consider another variant of our system, in which there is a bulk fluid of finite thickness resting on a solid substrate, and is covered by a fluid membrane at the top surface containing self-propelled particles attached to the membrane. How the order of the polar flock couples with the membrane undulations, and how this depends on the bulk fluid thickness, is an interesting, and completely open, question.

Another interesting extension would be to consider a variant of our system, in which the bulk fluid above has additional properties, e.g., if it is anisotropic or has long ranged correlations. This could be achieved if the passive bulk fluid is replaced, e.g., by a nematic liquid crystal [54]. The special ordering direction of the bulk nematic will be system specific (i.e., it will depend on the active particles, the nematic material used, and properties of the substrate, as well as (possibly) temperature and pressure. It will not, in general, be parallel to the direction of self-propulsion in the active fluid layer. Indeed, in some cases, it may even be along the direction orthogonal to the active fluid layer ( the $z$-direction in our geometry). Similarly, one might also extend this study by replacing the isotropic bulk fluid by a bulk smectic A or $\mathrm{C}$ liquid crystal. With a bulk smectic too, there could be competition between the in-plane self-propulsion direction in the active fluid layer and the alignment direction of the smectic molecules. How all these different possibilities conspire with the activity to produce a steady state of some type is an unresolved but open question.

Acknowledgements: One of us (AB) thanks the SERB, DST (India) for partial financial support through the MATRICS scheme [file no.: MTR/2020/000406]. NS is partially supported by Netherlands Organization for Scientific Research (NWO), through the Vidi grant No. 2016/N/00075794. We thank S. Ramaswamy for sharing reference 44] with us. NS thanks Institut Curie and MPIPKS for their support through postdoctoral fellowships while some of this work was being done. AB thanks the MPIPKS, Dresden for their hospitality, and their support through their Visitors' Program, while a portion of this work was underway. JT likewise thanks the MPIPKS for their hospitality, and their support through the Martin Gutzwiller Fellowship, and the Higgs Center of the University of Edinburgh for their support with a Higgs Fellowship.

\section{Appendix A: Relating the 3D bulk fluid flow to the surface velocity field}

The 3D bulk passive fluid satisfies the Stoke's equation

$$
\eta \nabla_{3}^{2} \mathbf{v}=\nabla_{3} \Pi
$$

where $\nabla_{3}$ is the $3 \mathrm{D}$ gradient operator, and $\Pi$ is the hydrostatic pressure associated with the bulk fluid in the $z>0$ region. We impose 3D incompressibility for this fluid, so that $\nabla_{3} \cdot \mathbf{v}=0$. Therefore, taking the divergence of A1 implies

$$
\nabla_{3}^{2} \Pi=0
$$

Taking the Laplacian of A1 and using (A2) then implies

$$
\nabla_{3}^{4} v_{\alpha}=0
$$

We now look for plane wave solutions of this equation; that is, solutions of the form

$$
\mathbf{v}\left(\mathbf{r}_{\perp}, z\right)=\mathbf{V}(z) \exp \left(i \mathbf{q} \cdot \mathbf{r}_{\perp}\right)
$$

where $\mathbf{q}$ is a two-dimensional in-plane Fourier wavevector and $\mathbf{r}_{\|} \equiv(x, y, 0)$ is the projection of $\mathbf{r}$ onto the plane of the surface.

We likewise assume that the velocity $\mathbf{v}_{s}(x, y)$ on the surface (which by assumption is parallel to the surface) also takes a plane wave form:

$$
\mathbf{v}_{s}\left(\mathbf{r}_{\perp}\right)=\mathbf{v}_{s}(\mathbf{q}) \exp \left(i \mathbf{q} \cdot \mathbf{r}_{\perp}\right)
$$


The general solution of this equation $\mathrm{A} 3$ of the form (A4) that vanishes as $z \rightarrow \infty$ is

$$
\mathbf{v}\left(\mathbf{r}_{\perp}, z\right)=\left(\mathbf{w}_{1}(\mathbf{q})+\mathbf{w}_{2}(\mathbf{q}) z\right) \exp (-q z) \exp \left(i \mathbf{q} \cdot \mathbf{r}_{\perp}(\mathrm{A} 6)\right.
$$

The boundary condition we impose on the $3 \mathrm{D}$ passive fluid at the fluid-solid interface is $v_{z}(z=0)=0$ and $\mathbf{v}_{\perp}(x, y, z=0)=\mathbf{v}_{s}(x, y)$, where $\perp$ denotes components of the velocity parallel to the fluid-solid interface, and

These boundary conditions entirely determine $\mathbf{w}_{1}$ in equation $\mathrm{A6}$ :

$$
\mathbf{w}_{1}(\mathbf{q})=\mathbf{v}_{s}(\mathbf{q})
$$

which implicitly fixes $\mathrm{w}_{1 z}=0$.

To determine $\mathbf{w}_{2}$, we take the curl of A1 to eliminate the pressure $\Pi_{3}$. This gives:

$$
\nabla_{3} \times \nabla_{3}^{2} \mathbf{v}=\mathbf{0}
$$

The Laplacian of our velocity field $\sqrt{\mathrm{A} 6}$ is

$$
\nabla_{3}^{2} \mathbf{v}=\mathbf{w}_{1} \nabla_{3}^{2}\left[\exp (-q z) \exp \left(i \mathbf{q} \cdot \mathbf{r}_{\perp}\right)\right]+\mathbf{w}_{2} \nabla_{3}^{2}\left[z \exp (-q z) \exp \left(i \mathbf{q} \cdot \mathbf{r}_{\perp}\right)\right]=-2 q \mathbf{w}_{2} \exp (-q z) \exp \left(i \mathbf{q} \cdot \mathbf{r}_{\perp}\right)
$$

Inserting this into $\mathrm{A} 8$ gives

$$
(-q \hat{\mathbf{z}}+i \mathbf{q}) \times \mathbf{w}_{2}=\mathbf{0}
$$

Taking the dot product of this with $\mathbf{q}$ (which we remind the reader lies in the plane of the surface, and, hence, perpendicular to $\hat{\mathbf{z}}$ ) implies

$$
\mathbf{q} \cdot\left(\hat{\mathbf{z}} \times \mathbf{w}_{2}\right)=0
$$

Using the cyclic properties of triple products, this in turn implies

$$
\hat{\mathbf{z}} \cdot\left(\mathbf{q} \times \mathbf{w}_{2}\right)=0 .
$$

Since $\hat{\mathbf{z}} \cdot \mathbf{q} \times \hat{\mathbf{z}}=0$, this only imposes a condition on the in-plane components $\mathbf{w}_{2} \perp$ of $\mathbf{w}$ :

$$
\mathbf{q} \times \mathbf{w}_{2 \perp}=\mathbf{0},
$$

the most general solution of which is

$$
\mathbf{w}_{2 \perp}=\mathbf{q} \phi
$$

To determine the remaining unknown quantities, which are $\phi$ and $\mathrm{w}_{2 z}$, we insert our expression $\mathrm{A} 6$ for $\mathbf{v}$ into the incompressibility condition:

$$
\boldsymbol{\nabla}_{3} \cdot \mathbf{v}=\left[i\left(\mathbf{q} \cdot \mathbf{v}_{s}\right)+\mathrm{w}_{2 z}\right] \exp (-q z) \exp \left(i \mathbf{q} \cdot \mathbf{r}_{\perp}\right)+\left(i \mathbf{q} \cdot \mathbf{w}_{2}-q \mathrm{w}_{2 z}\right) z \exp (-q z) \exp \left(i \mathbf{q} \cdot \mathbf{r}_{\perp}\right)=0
$$

where we have replaced $\mathbf{w}_{1}$ with $v_{s}$ everywhere it appears.

To satisfy this equation, the coefficients of both $\exp (-q z) \exp \left(i \mathbf{q} \cdot \mathbf{r}_{\perp}\right)$ and $z \exp (-q z) \exp \left(i \mathbf{q} \cdot \mathbf{r}_{\perp}\right)$ must vanish. The former condition implies

$$
\mathrm{w}_{2 z}=-i \mathbf{q} \cdot \mathbf{v}_{s}
$$

which can be used in the latter to give

$$
i \mathbf{q} \cdot \mathbf{w}_{2}=-i q\left(\mathbf{q} \cdot \mathbf{v}_{s}\right) .
$$

Using A14 to rewrite this equation in terms of $\phi$, and solving for $\phi$, gives

$$
\phi=-\frac{\mathbf{q} \cdot \mathbf{v}_{s}}{q},
$$

which in turn implies via A14 that

$$
\mathbf{w}_{\perp}=-\hat{q} \mathbf{q} \cdot \mathbf{v}_{s} .
$$

Now using A7, A16, and (A19) in A6, we obtain:

$$
\mathbf{v}\left(\mathbf{r}_{\perp}, z, t\right)=\left[\mathbf{v}_{s}-z\left(\mathbf{q} \cdot \mathbf{v}_{s}\right)(\hat{\mathbf{q}}+i \hat{\mathbf{z}})\right] e^{-q z+i \mathbf{q} \cdot \mathbf{r}_{\perp}} .
$$

We have so far focused on solutions to the Stokes' equation A3 that are single plane waves. The most general solution, of course, can be obtained by summing up plane waves with all possible values of the wavevector $\mathbf{q}$. This gives

$$
\mathbf{v}\left(\mathbf{r}_{\perp}, z, t\right)=\sum_{\mathbf{q}}\left[\mathbf{v}_{s}(\mathbf{q})-z\left(\mathbf{q} \cdot \mathbf{v}_{s}(\mathbf{q})\right)(\hat{\mathbf{q}}+i \hat{\mathbf{z}})\right] e^{-q z+i \mathbf{q} \cdot \mathbf{r}_{\perp}}
$$




\section{Appendix B: Derivation of the stability condition}

We will now prove, in two steps, that the conditions $c_{0}^{2}>0, \gamma>0$, and $\phi$ sufficiently small imply that $\operatorname{Im}(\Upsilon(\theta))<0$ for all $\theta$. First, we will show that $\operatorname{Im}(\Upsilon(\theta=\pi / 2))<0$. Then we will show that $\operatorname{Im}(\Upsilon(\theta))$ cannot equal 0 for any value of $\theta$. Since $\operatorname{Im}(\Upsilon(\theta))$ is obviously a continuous function of $\theta$, this implies that it can never become positive, since to get from its negative value at $\theta=\pi / 2$ to a positive value, it would first have to pass through 0 .

We begin by showing $\operatorname{Im}(\Upsilon(\theta=\pi / 2))<0$. Setting $\theta=\pi / 2$ in $\mathrm{IV} .5$ gives

$$
\Upsilon(\pi / 2)^{2}+2 i \gamma \Upsilon(\pi / 2)-c_{0}^{2}=0
$$

which is readily solved to give

$$
\Upsilon(\pi / 2)=-i \gamma \pm \sqrt{c_{0}^{2}-\gamma^{2}}
$$

If $c_{0}^{2}$ and $\gamma$ are both $>0$, then the magnitude of the argument of the square root in this expression is clearly less than $\gamma$. Hence, even if $\gamma^{2}>c_{0}^{2}$, so that the argument of the square root is negative, making the square root itself purely imaginary, it can only add to the imaginary part of $\Upsilon$ a contribution smaller in magnitude than $-i \gamma$; hence, it cannot make the imaginary part positive. If $\gamma<c_{0}^{2}$, things are even simpler: the square root in $\mathrm{B} 2$ is real, and $\operatorname{Im}(\Upsilon(\theta=\pi / 2))=-\gamma$, which is $<0$ if $\gamma>0$.

So our stability conditions $c_{0}^{2}>0, \gamma>0$ imply that $\operatorname{Im}(\Upsilon(\theta=\pi / 2))<0$. We now complete our proof that $\operatorname{Im}(\Upsilon(\theta))<0$ for all $\theta$ by showing that $\operatorname{Im}(\Upsilon(\theta)) \neq 0$ for any value of $\theta$. We will prove this by contradiction: assume that $\operatorname{Im}(\Upsilon(\theta))=0$ at some value of $\theta$. Then at that value of $\theta, \Upsilon(\theta))$ is real. Therefore, the imaginary part of (IV.5) reads

$$
\Upsilon \gamma\left(1+\sin ^{2} \theta\right)-\gamma v_{\rho} \cos \theta\left(1+\varphi \sin ^{2} \theta\right)=0
$$

which can be solved for $\Upsilon$ :

$$
\Upsilon=v_{\rho} \cos \theta\left(\frac{1+\varphi \sin ^{2} \theta}{1+\sin ^{2} \theta}\right)
$$

Inserting ( $\mathrm{B} 4)$ into the real part of $(\overline{\mathrm{IV} .5})$ then implies

$$
v_{\rho}^{2} \cos ^{2} \theta\left(\frac{1+\varphi \sin ^{2} \theta}{1+\sin ^{2} \theta}\right)^{2}-v_{\rho}\left(v_{\rho}+v_{p}\right) \cos ^{2} \theta\left(\frac{1+\varphi \sin ^{2} \theta}{1+\sin ^{2} \theta}\right)+v_{p} v_{\rho} \cos ^{2} \theta-c_{0}^{2} \sin ^{2} \theta=0 .
$$

After a bit of algebra, and using $\varphi=1-\frac{\gamma_{\rho}}{\gamma}$, this can be rewritten

$$
\left[\left(\frac{v_{\rho} \gamma_{\rho}}{\gamma}\right) \cos ^{2} \theta\left[v_{p}-v_{\rho}+\left(v_{p}-\varphi v_{\rho}\right) \sin ^{2} \theta\right]-c_{0}^{2}\left(1+\sin ^{2} \theta\right)^{2}\right] \sin ^{2} \theta=0
$$

Our original assumption that $\operatorname{Im}(\Upsilon(\theta))=0$ can therefore only be satisfied if $(\mathrm{B} 6)$ has a solution for some real $\theta$ between $-\pi$ and $\pi$. To say this another way, our system will be stable if $(\mathrm{B} 6)$ has no solution for any real $\theta$.
Since, in that range of $\theta, \sin ^{2} \theta \neq 0$, we can divide $\mathrm{B} 6$ by $\sin ^{2} \theta$. Doing so, and reorganizing a bit further, we can rewrite $(\mathrm{B} 6)$ in dimensionless form as

$$
\left(\frac{m^{2} \gamma_{\rho}}{\gamma}\right) \cos ^{2} \theta\left[\varpi-1+(\varpi-\varphi) \sin ^{2} \theta\right]=\left(1+\sin ^{2} \theta\right)^{2}
$$

where we have defined the "Mach number"

$$
m \equiv \frac{v_{\rho}}{c_{0}},
$$

and the speed ratio

$$
\varpi \equiv \frac{v_{p}}{v_{\rho}}
$$

This equation clearly has no solution for real $\theta$, implying that the system is stable, if the left hand side of the equation is bounded above by 1 , since the right hand side is clearly bounded below by 1 .

We can derive such a bound by using $\cos ^{2} \theta \leq 1$, $\sin ^{2} \theta \leq 1, \varpi-1 \leq|\varpi-1|$, and $\varphi=1-\frac{\gamma_{\rho}}{\gamma}$ to show 
that the left hand side (LHS) obeys

$$
\operatorname{LHS}<\left(\frac{m^{2} \gamma_{\rho}}{\gamma}\right)\left[2|\varpi-1|+\frac{\gamma_{\rho}}{\gamma}\right]
$$

The right hand side of equation $(\mathrm{B} 10)$ is a quadratic function of the ratio $\frac{\gamma_{\rho}}{\gamma}$. Requiring that it be less than 1 leads to the bounds on that ratio:

$$
-|\varpi-1|-\sqrt{(\varpi-1)^{2}+\frac{1}{m^{2}}}<\frac{\gamma_{\rho}}{\gamma}<-|\varpi-1|+\sqrt{(\varpi-1)^{2}+\frac{1}{m^{2}}}
$$

It is easy to see that this condition can always be satisfied for sufficiently small $\gamma_{\rho}$; in particular, the allowed region always includes $\gamma_{\rho}=0$.

Note that we have shown that the condition (B11) is sufficient for stability; however, it is by no means necessary.

Nonetheless, having such a sufficient condition proves that the ordered state in this system, unlike that of "wet" systems, can be stable. More specifically, as long as $c_{0}^{2}$ and $\gamma$ are positive, there is always a window of stability at sufficiently small $\gamma_{\rho}$.

At $\theta=0, \pi, \operatorname{Im} \Upsilon(\theta)=0$; however, there are stabilizing $\mathcal{O}\left(q^{2}\right)$ damping terms that have been neglected in the previous discussion. For these two special directions of propagation, the dynamics of the density fluctuations $\delta \rho$ decouple, up to $\mathcal{O}(q)$, from those of $p_{y}$. Hence the density fluctuations are not damped at this order. This is not really a problem, as there are diffusive damping terms at $\mathcal{O}\left(q^{2}\right)$ which provide damping to the density fluctuations. These damping terms are the $\left[D_{\rho} \nabla_{s}^{2}+D_{\rho 2} \partial_{x}^{2}\right] \delta \rho$ terms, which, for $\theta=0, \pi$ in Fourier space, give $-\left(D_{\rho}+D_{\rho 2}\right) q^{2} \delta \rho$ and ultimately damp the density fluctuations at $\theta=0, \pi$. In fact, this decoupling of $\delta \rho$ and $p_{y}$ at $\mathcal{O}(q)$ at $\theta=0, \pi$ is the origin of the special behaviors of the scaling functions at those points.

Q.E.D.

\section{Appendix C: Evaluation of the integral for the equal time correlation function}

In this appendix, we evaluate the integral

$$
I \equiv \int_{-\infty}^{\infty} \frac{\left(s+\psi\left(\varphi, \theta_{\mathbf{q}}\right)^{2} d s\right.}{\left(s-\psi_{+}\left(\varphi, \theta_{\mathbf{q}}\right)\right)^{2}\left(s-\psi_{-}\left(\varphi, \theta_{\mathbf{q}}\right)\right)^{2}+s^{2}}
$$

in equation (V.16), which arises in the calculation of the equal time correlation function $C_{p p}(\mathbf{q}) \equiv\left\langle\left|p_{y}(\mathbf{q}, t)\right|^{2}\right\rangle$, is given by $I=\pi$, independent of all parameters, and $\theta_{\mathbf{q}}$.

This proves to be true if the two quantities

$$
\psi_{ \pm}\left(\theta_{\mathbf{q}}\right) \equiv \frac{c_{ \pm}\left(\theta_{\mathbf{q}}\right)-v_{\rho} \cos \theta_{\mathbf{q}} \Xi\left(\varphi, \theta_{\mathbf{q}}\right)}{\Psi\left(1, \theta_{\mathbf{q}}\right)}
$$

obey $\psi_{+}\left(\theta_{\mathbf{q}}\right)>0$, and $\psi_{-}\left(\theta_{\mathbf{q}}\right)<0$. We will now show that $\psi_{+}\left(\theta_{\mathbf{q}}\right)$, and $\psi_{-}\left(\theta_{\mathbf{q}}\right)$ do indeed obey these inequalities whenever the system is stable. We will then show that when these inequalities are satisfied, $I=\pi$.

We begin by showing that $\psi_{+}\left(\theta_{\mathbf{q}}\right)>0$, and $\psi_{-}\left(\theta_{\mathbf{q}}\right)<$ 0 . This can be shown by first rewriting C2 using our expression $\mathrm{V} .4$ for the sound speeds $c_{ \pm}\left(\theta_{\mathbf{q}}\right)$ :

$$
\psi_{ \pm}\left(\theta_{\mathbf{q}}\right)=\left\{\left(\frac{v_{\rho}(1-2 \Xi)+v_{p}}{2}\right) \cos \theta_{\mathbf{q}} \pm \sqrt{\frac{1}{4}\left(v_{\rho}-v_{p}\right)^{2} \cos ^{2} \theta_{\mathbf{q}}+c_{0}^{2} \sin ^{2} \theta_{\mathbf{q}}}\right\} / \Psi\left(1, \theta_{\mathbf{q}}\right)
$$

Since $\Psi\left(1, \theta_{\mathbf{q}}\right)=\gamma\left(1+\sin ^{2} \theta\right)$ is always positive if $\gamma>0$ (as it must be for stability), we will clearly have $\psi_{+}\left(\theta_{\mathbf{q}}\right)>0$, and $\psi_{-}\left(\theta_{\mathbf{q}}\right)<0$ if the magnitude of the square root in $\mathrm{C} 3$ is greater than the magnitude of the term $\left(\frac{v_{\rho}(1-2 \Xi)+v_{p}}{2}\right) \cos \theta_{\mathbf{q}}$ in that equation. This is equivalent to the argument of that square root being big- ger than the square of $\left(\frac{v_{\rho}(1-2 \Xi)+v_{p}}{2}\right) \cos \theta_{\mathbf{q}}$. This leads to the condition

$\frac{1}{4}\left(v_{\rho}-v_{p}\right)^{2} \cos ^{2} \theta_{\mathbf{q}}+c_{0}^{2} \sin ^{2} \theta_{\mathbf{q}}>\left[\left(\frac{v_{\rho}(1-2 \Xi)+v_{p}}{2}\right) \cos \theta_{\mathbf{q}}\right]^{2}$ 
as a necessary and sufficient condition for making $\psi_{+}\left(\theta_{\mathbf{q}}\right)>0$, and $\psi_{-}\left(\theta_{\mathbf{q}}\right)<0$.
After a little (!) algebra, and using the fact that

$$
\Xi(\Phi, \theta) \equiv \frac{\Psi(\Phi, \theta)}{\Psi(1, \theta)}=\frac{1+\Phi \sin ^{2} \theta}{1+\sin ^{2} \theta},
$$

this can be rewritten as

$$
\left(\frac{v_{\rho} \gamma_{\rho}}{\gamma}\right) \cos ^{2} \theta\left[v_{p}-v_{\rho}+\left(v_{p}-\varphi v_{\rho}\right)\right]<c_{0}^{2}\left(1+\sin ^{2} \theta\right)^{2}
$$

which the alert reader will note is precisely the same as our stability condition (IV.6). So, if our system is stable, $\psi_{+}\left(\theta_{\mathbf{q}}\right)>0$, and $\psi_{-}\left(\theta_{\mathbf{q}}\right)<0$.

Now we turn to the evaluation of the integral $I$ equation C1. This can clearly be rewritten as

$$
I=I_{1}+2 \psi I_{2}+\psi^{2} I_{3}
$$

with

$$
I_{1}=\int_{-\infty}^{\infty} \frac{s^{2} d s}{\left(s-\psi_{+}\left(\theta_{\mathbf{q}}\right)\right)^{2}\left(s-\psi_{-}\left(\theta_{\mathbf{q}}\right)\right)^{2}+s^{2}}
$$

$$
I_{2}=\int_{-\infty}^{\infty} \frac{s d s}{\left(s-\psi_{+}\left(\theta_{\mathbf{q}}\right)\right)^{2}\left(s-\psi_{-}\left(\theta_{\mathbf{q}}\right)\right)^{2}+s^{2}}
$$

and

$$
I_{3}=\int_{-\infty}^{\infty} \frac{d s}{\left(s-\psi_{+}\left(\theta_{\mathbf{q}}\right)\right)^{2}\left(s-\psi_{-}\left(\theta_{\mathbf{q}}\right)\right)^{2}+s^{2}} .
$$

We will now evaluate each of these using the method of partial fractions. This begins with the identity

$$
\frac{1}{\left(s-\psi_{+}\left(\theta_{\mathbf{q}}\right)\right)^{2}\left(s-\psi_{-}\left(\theta_{\mathbf{q}}\right)\right)^{2}+s^{2}}=\frac{1}{2 i s}\left[\frac{1}{\left(s-\psi_{+}\left(\theta_{\mathbf{q}}\right)\right)\left(s-\psi_{-}\left(\theta_{\mathbf{q}}\right)\right)-i s}-\frac{1}{\left(s-\psi_{+}\left(\theta_{\mathbf{q}}\right)\right)\left(s-\psi_{-}\left(\theta_{\mathbf{q}}\right)\right)+i s}\right] .
$$

Using this, we can rewrite $I_{1}$ C8 as

$$
I_{1}=\int_{-\infty}^{\infty} \frac{s^{2} d s}{\left(s-\psi_{+}\left(\theta_{\mathbf{q}}\right)\right)^{2}\left(s-\psi_{-}\left(\theta_{\mathbf{q}}\right)\right)^{2}+s^{2}}=\frac{1}{2 i}\left(I_{1-}-I_{1+}\right)
$$

where we have defined

$$
I_{1 \pm} \equiv \int_{-\infty}^{\infty} \frac{s d s}{\left(s-\psi_{+}\left(\theta_{\mathbf{q}}\right)\right)\left(s-\psi_{-}\left(\theta_{\mathbf{q}}\right)\right) \pm i s}
$$

We will now evaluate $I_{1 \pm}$; in particular, we will show that both are independent of $\psi_{ \pm}$.

Consider first $I_{1+}$, and the poles of its integrand in the complex $s$ plane. There are obviously two of these, which we can write as

$$
s_{1,2}=r_{1,2} e^{i \phi_{1,2}}
$$

with $r_{1,2}$ both real and positive, and $\phi_{1,2}$ real. Inspection of the denominator in $\mathrm{C} 13$ shows that these must obey

$$
r_{1} e^{i \phi_{1}}+r_{2} e^{i \phi_{2}}=\psi_{+}\left(\theta_{\mathbf{q}}\right)+\psi_{-}\left(\theta_{\mathbf{q}}\right)-i
$$

and

$$
r_{1} r_{2} e^{i\left(\phi_{1}+\phi_{2}\right)}=\psi_{+}\left(\theta_{\mathbf{q}}\right) \psi_{-}\left(\theta_{\mathbf{q}}\right) .
$$

Since $\psi_{+}>0$ and $\psi_{-}<0, \psi_{+} \psi_{-}<0$. Using this fact in (C16), and recalling that $r_{1}$ and $r_{2}$ are both real and positive, we see that

$$
\phi_{1}+\phi_{2}=\pi
$$

from which it immediately follows that

$$
s_{2}=r_{2} e^{i\left(\pi-\phi_{1}\right)}=-r_{2} e^{-i \phi_{1}} .
$$

Note that $\mathrm{C} 18$ implies that $s_{1}$ and $s_{2}$ lie on the same side of the real axis. Using (C18) in C15 gives

$$
r_{1} e^{i \phi_{1}}-r_{2} e^{-i \phi_{1}}=\psi_{+}\left(\theta_{\mathbf{q}}\right)+\psi_{-}\left(\theta_{\mathbf{q}}\right)-i
$$


Taking the imaginary part of this expression gives

$$
\left(r_{1}+r_{2}\right) \sin \phi_{1}=-1
$$

which implies that $\sin \phi_{1}<0$, which in turn implies that $s_{1}$, and, hence, $s_{2}$, both lie in the lower half plane.

Then if we consider the contour $\mathcal{C}$ in the complex $s$ plane consisting of the real axis plus the infinite semicircle above the real axis connecting its ends, it follows from the absence of poles in the upper half plane that

$$
\oint_{\mathcal{C}} \frac{s d s}{\left(s-\psi_{+}\left(\theta_{\mathbf{q}}\right)\right)\left(s-\psi_{-}\left(\theta_{\mathbf{q}}\right)\right)+i s}=0 .
$$

The integral in this expression can be written as

$$
\oint_{\mathcal{C}} \frac{s d s}{\left(s-\psi_{+}\left(\theta_{\mathbf{q}}\right)\right)\left(s-\psi_{-}\left(\theta_{\mathbf{q}}\right)\right)+i s}=I_{1+}+\int_{\mathcal{S}} \frac{s d s}{\left(s-\psi_{+}\left(\theta_{\mathbf{q}}\right)\right)\left(s-\psi_{-}\left(\theta_{\mathbf{q}}\right)\right)+i s}
$$

where the contour $\mathcal{S}$ is the aforementioned infinite semicircle. Since that semicircle is infinite, $\psi_{ \pm}$and $i s$ can be neglected in the denominator of the integrand relative to $s^{2}$. Thus we have

$\int_{\mathcal{S}} \frac{s d s}{\left(s-\psi_{+}\left(\theta_{\mathbf{q}}\right)\right)\left(s-\psi_{-}\left(\theta_{\mathbf{q}}\right)\right)+i s}=\int_{\mathcal{S}} \frac{s d s}{s^{2}}=\int_{\mathcal{S}} \frac{d s}{s}=i \pi$.

Using this in $\mathrm{C} 22$ and using the result in $\mathrm{C} 21$ gives

$$
I_{+}=-i \pi
$$

It is straightforward to repeat this reasoning for $I_{-}$; in that case, both poles lie in the upper half plane, and the semicircle must lie in the lower half plane. We obtain

$$
I_{-}=i \pi
$$

Using $(\mathrm{C} 24)$ and $(\mathrm{C} 25)$ in $\mathrm{C} 12$, we obtain

$$
I_{1}=\int_{-\infty}^{\infty} \frac{s^{2} d s}{\left(s-\psi_{+}\left(\theta_{\mathbf{q}}\right)\right)^{2}\left(s-\psi_{-}\left(\theta_{\mathbf{q}}\right)\right)^{2}+s^{2}}=\pi .
$$

as claimed earlier.

Similar reasoning can be applied to $I 2$ and $I 3$. For $I 2$, we get

$$
I_{2}=\int_{-\infty}^{\infty} \frac{s d s}{\left(s-\psi_{+}\left(\theta_{\mathbf{q}}\right)\right)^{2}\left(s-\psi_{-}\left(\theta_{\mathbf{q}}\right)\right)^{2}+s^{2}}=\frac{1}{2 i}\left(I_{2-}-I_{2+}\right)
$$

where we have defined

$$
I_{2 \pm} \equiv \int_{-\infty}^{\infty} \frac{d s}{\left(s-\psi_{+}\left(\theta_{\mathbf{q}}\right)\right)\left(s-\psi_{-}\left(\theta_{\mathbf{q}}\right)\right) \pm i s} .
$$

Proceeding as we did above for $I_{1}$, we find

$$
\oint_{\mathcal{C}} \frac{d s}{\left(s-\psi_{+}\left(\theta_{\mathbf{q}}\right)\right)\left(s-\psi_{-}\left(\theta_{\mathbf{q}}\right)\right)+i s}=I_{2+}+\int_{\mathcal{S}} \frac{d s}{\left(s-\psi_{+}\left(\theta_{\mathbf{q}}\right)\right)\left(s-\psi_{-}\left(\theta_{\mathbf{q}}\right)\right)+i s}=0 .
$$

Again using the fact that semicircle is infinite, we can

again neglect $\psi_{ \pm}$and $i s$ in the denominator of the integrand relative to $s^{2}$. Thus we have

$$
\int_{\mathcal{S}} \frac{d s}{\left(s-\psi_{+}\left(\theta_{\mathbf{q}}\right)\right)\left(s-\psi_{-}\left(\theta_{\mathbf{q}}\right)\right)+i s}=\int_{\mathcal{S}} \frac{d s}{s^{2}}=\lim _{R \rightarrow \infty} \int_{\mathcal{S}} \frac{d s}{s^{2}}=\lim _{R \rightarrow \infty}\left(1-e^{-i \pi}\right) / R=0
$$

Thus we find

$$
I_{2+}=0 \text {. }
$$

Similar reasoning shows that

$$
I_{2-}=0
$$


as well. Taking these two results C31 and C31 together in $\mathrm{C} 27$ gives

$$
I_{2}=0
$$

Finally, applying this partial fraction approach to $I_{3}$, we obtain

$$
I_{3}=\left(\frac{1}{2 i}\right)\left(I_{3-}-I_{3+}\right)
$$

where we have defined

$$
I_{3 \pm} \equiv \int_{-\infty}^{\infty} \frac{d s}{(s-i \epsilon)\left[\left(s-\psi_{+}\left(\theta_{\mathbf{q}}\right)\right)\left(s-\psi_{-}\left(\theta_{\mathbf{q}}\right)\right) \pm i s\right]}
$$

and we have shifted the pole of $\frac{1}{s}$ above the real axis by a small amount $\epsilon$, which we will take to zero at the end of our calculation. It is straightforward to check that choosing to move the pole below the real axis by a small amount $\epsilon$ leads to exactly the same final answer for $I_{3}$.

Proceeding with the choice of moving the pole above the axis, we note that, for this choice, all of the poles in the integrand for $I_{3-}$ lie in the upper half plane. Therefore, evaluating the integral by closing the contour in the lower half plane (which we can do with impunity, since the integrand vanishes like $\frac{1}{s^{2}}$ as $|s| \rightarrow \infty$, which implies that the semi-infinite semi-circle with we close the contour contributes nothing to the integral), we find that $I_{3-}=0$.

On the other hand, for $I_{3+}$, there is a single pole at $s=$ $i \epsilon$ and the upper half plane, and two poles (as discussed earlier) in the lower half plane. Making the easy choice of closing the contour in the upper half plane, and taking the limit $\epsilon \rightarrow 0$, we easily find

$$
I_{3+}=\frac{2 \pi i}{\psi_{+} \psi_{-}}
$$

Using this in (C34, we obtain our final result for $I_{3}$ :

$$
I_{3}=-\frac{\pi}{\psi_{+} \psi_{-}} .
$$

Using this result C36 and our earlier results $I_{1}=\pi$ and $I_{2}=0$ in our expression C7 for the integral $I$ gives

$$
I=\pi\left(1-\frac{\psi^{2}}{\psi_{+} \psi_{-}}\right)
$$

Using our expressions (C3) and (V.18) for $\psi_{ \pm}$and $\psi$ in this result, we obtain, after a little (!) algebra, our final expression

$$
I=\pi\left(1+\left(\frac{\gamma_{\rho}}{\gamma}\right)^{2} \frac{m^{2} \sin ^{2} \theta \cos ^{2} \theta}{\left\{\left(1+\sin ^{2} \theta\right)^{2}-m^{2}\left[\varpi-1+(\varpi-\varphi) \sin ^{2} \theta\right] \cos ^{2} \theta\right\}}\right) .
$$

\section{Appendix D: Evaluation of the non-local kernels}

Introducing an exponential UV cutoff, we can write

$$
\mathcal{K}_{p p}\left(\mathbf{r}_{\|}\right)=\int \frac{d^{2} q}{(2 \pi)^{2}}\left(\frac{q^{2}+q_{y}^{2}}{q}\right) e^{i \mathbf{q} \cdot \mathbf{r}_{\|}-a q}=-\left(\partial_{x}^{2}+2 \partial_{y}^{2}\right) \phi\left(\mathbf{r}_{\|}\right)
$$

where $a \equiv 2 \pi / \Lambda$, with $\Lambda$ being the ultraviolet cutoff, and

$$
\begin{aligned}
\phi\left(\mathbf{r}_{\|}\right) & =\int \frac{d^{2} q}{(2 \pi)^{2}}\left(\frac{1}{q}\right) e^{i \mathbf{q} \cdot \mathbf{r}_{\|}-a q}=\frac{1}{4 \pi^{2}} \int_{0}^{2 \pi} d \theta \int_{0}^{\infty} \exp \left(\left[i r_{\perp} \cos \theta-a\right] q\right) d q=\frac{1}{4 \pi^{2}} \int_{0}^{2 \pi} \frac{d \theta}{a-i r_{\perp} \cos \theta} \\
& =\frac{1}{4 \pi^{2}} \int_{0}^{2 \pi} \frac{\left(a+i r_{\perp} \cos \theta\right) d \theta}{a^{2}+r_{\perp}^{2} \cos ^{2} \theta}
\end{aligned}
$$

The $\cos \theta$ term in the numerator is odd under $\theta \rightarrow \pi-\theta$, so its integral vanishes. The remainder of the integral is even under $\theta \rightarrow \pi-\theta$, so we can replace its integral with 
twice the integral from $-\pi / 2$ to $\pi / 2$. Doing so, we get

$$
\phi\left(\mathbf{r}_{\|}\right)=\frac{a}{2 \pi^{2}} \int_{-\pi / 2}^{\frac{\pi}{2}} \frac{d \theta}{a^{2}+r_{\perp}^{2} \cos ^{2} \theta} .
$$

The integral can be done straightforwardly with the substitution $u=\tan \theta$, with the result

$$
\phi\left(\mathbf{r}_{\|}\right)=\frac{1}{2 \pi \sqrt{a^{2}+r_{\perp}^{2}}} .
$$

Inserting $\mathrm{C} 4$ into the last equality of $\mathrm{C} 1$ and taking the derivatives gives

$$
\mathcal{K}_{p p}\left(\mathbf{r}_{\|}\right)=\left(\frac{3 a^{2}-3 y^{2}}{2 \pi\left(a^{2}+r_{\perp}^{2}\right)^{5 / 2}}\right) .
$$

Likewise,

$$
\mathcal{K}_{p p \rho}\left(\mathbf{r}_{\|}\right)=\left(\frac{3 a^{2}-3 x^{2}}{2 \pi\left(a^{2}+r_{\perp}^{2}\right)^{5 / 2}}\right) .
$$

$$
\begin{aligned}
& \int_{0}^{L_{y}} d y^{\prime} \frac{\left|\mathbf{r}-\mathbf{r}^{\prime}\right|}{\left(\mathbf{r}-\mathbf{r}^{\prime}\right)^{2}+\left(x-x^{\prime}\right)^{2}} \\
& \times\left[1-\frac{\left(v_{p}-v_{\rho}\right)^{2}\left(y-y^{\prime}\right)}{\left(\mathbf{r}-\mathbf{r}^{\prime}\right)^{2}+\left(x-x^{\prime}\right)^{2}} \frac{\gamma_{\rho} v_{\rho}}{\gamma c_{0}^{2}}-\left(\frac{v_{\rho} \gamma_{\rho}}{c_{0} \gamma}\right)^{2} \frac{\left(y-y^{\prime}\right)^{2}\left(x-x^{\prime}\right)^{2}}{\left\{\left(\mathbf{r}-\mathbf{r}^{\prime}\right)^{2}+\left(x-x^{\prime}\right)^{2}\right\}^{2}}\right]^{-1}=I_{4}+I_{5}+I_{6},
\end{aligned}
$$

where we have defined

$$
\begin{aligned}
I_{4} \equiv & \int_{0}^{y-\tilde{C} L_{x}} d y^{\prime} \frac{\left|\mathbf{r}-\mathbf{r}^{\prime}\right|}{\left(\mathbf{r}-\mathbf{r}^{\prime}\right)^{2}+\left(x-x^{\prime}\right)^{2}} \\
& \times\left[1-\frac{\left(v_{p}-v_{\rho}\right)\left(y-y^{\prime}\right)^{2}}{\left(\mathbf{r}-\mathbf{r}^{\prime}\right)^{2}+\left(x-x^{\prime}\right)^{2}} \frac{\gamma_{\rho} v_{\rho}}{\gamma c_{0}^{2}}-\left(\frac{v_{\rho} \gamma_{\rho}}{c_{0} \gamma}\right)^{2} \frac{\left(y-y^{\prime}\right)^{2}\left(x-x^{\prime}\right)^{2}}{\left\{\left(\mathbf{r}-\mathbf{r}^{\prime}\right)^{2}+\left(x-x^{\prime}\right)^{2}\right\}^{2}}\right]^{-1}, \\
I_{5} \equiv & \int_{y-\tilde{C} L_{x}}^{y+\tilde{C} L_{x}} d y^{\prime} \frac{\left|\mathbf{r}-\mathbf{r}^{\prime}\right|}{\left(\mathbf{r}-\mathbf{r}^{\prime}\right)^{2}+\left(x-x^{\prime}\right)^{2}} \\
& \times\left[1-\frac{\left(v_{p}-v_{\rho}\right)\left(y-y^{\prime}\right)^{2}}{\left(\mathbf{r}-\mathbf{r}^{\prime}\right)^{2}+\left(x-x^{\prime}\right)^{2}} \frac{\gamma_{\rho} v_{\rho}}{\gamma c_{0}^{2}}-\left(\frac{v_{\rho} \gamma_{\rho}}{c_{0} \gamma}\right)^{2} \frac{\left(y-y^{\prime}\right)^{2}\left(x-x^{\prime}\right)^{2}}{\left\{\left(\mathbf{r}-\mathbf{r}^{\prime}\right)^{2}+\left(x-x^{\prime}\right)^{2}\right\}^{2}}\right]^{-1},
\end{aligned}
$$

and

$$
\begin{aligned}
I_{6} \equiv & \int_{y+\tilde{C} L_{x}}^{L_{y}} d y^{\prime} \frac{\left|\mathbf{r}-\mathbf{r}^{\prime}\right|}{\left(\mathbf{r}-\mathbf{r}^{\prime}\right)^{2}+\left(x-x^{\prime}\right)^{2}} \\
& \times\left[1-\frac{\left(v_{p}-v_{\rho}\right)\left(y-y^{\prime}\right)^{2}}{\left(\mathbf{r}-\mathbf{r}^{\prime}\right)^{2}+\left(x-x^{\prime}\right)^{2}} \frac{\gamma_{\rho} v_{\rho}}{\gamma c_{0}^{2}}-\left(\frac{v_{\rho} \gamma_{\rho}}{c_{0} \gamma}\right)^{2} \frac{\left(y-y^{\prime}\right)^{2}\left(x-x^{\prime}\right)^{2}}{\left\{\left(\mathbf{r}-\mathbf{r}^{\prime}\right)^{2}+\left(x-x^{\prime}\right)^{2}\right\}^{2}}\right]^{-1}
\end{aligned}
$$

Once again, we have chosen the constant $\tilde{C}$ to be sufficiently large compared to 1 that, throughout the regions 
of integration of $I_{4}$ and $I_{6},\left|y-y^{\prime}\right| \gg L_{x}$. Hence, in those regions of integration,

$$
\begin{aligned}
& \frac{\left|\mathbf{r}-\mathbf{r}^{\prime}\right|}{\left(\mathbf{r}-\mathbf{r}^{\prime}\right)^{2}+\left(x-x^{\prime}\right)^{2}} \approx \frac{1}{y-y^{\prime}}, \\
& \frac{\left(y-y^{\prime}\right)^{2}}{\left(x-x^{\prime}\right)^{2}+\left(\mathbf{r}-\mathbf{r}^{\prime}\right)^{2}} \approx 1, \\
& \frac{\left(y-y^{\prime}\right)^{2}\left(x-x^{\prime}\right)^{2}}{\left[\left(x-x^{\prime}\right)^{2}+\left(\mathbf{r}-\mathbf{r}^{\prime}\right)^{2}\right]^{2}} \ll 1 .
\end{aligned}
$$

This implies that

$I_{4} \approx\left[1-\frac{\left(v_{p}-v_{\rho}\right) \gamma_{\rho} v_{\rho}}{\gamma c_{0}^{2}}\right]^{-1} \int_{0}^{y-\tilde{C} L_{x}} \frac{d y^{\prime}}{y-y^{\prime}}=\ln \left(\frac{y}{\tilde{C} L_{x}}\right)\left[1-\frac{\left(v_{p}-v_{\rho}\right) \gamma_{\rho} v_{\rho}}{\gamma c_{0}^{2}}\right]^{-1}=\left\{\ln \left(\frac{y}{L_{x}}\right)-\ln \tilde{C}\right\}\left[1-\frac{\left(v_{p}-v_{\rho}\right) \gamma_{\rho} v_{\rho}}{\gamma c_{0}^{2}}\right]^{-1}$

Virtually identical reasoning can be applied to $I_{6}$, giv- $\quad$ ing the result

$$
I_{6}=\left\{\ln \left(\frac{L_{y}-y}{L_{x}}\right)-\ln \tilde{C}\right\}\left[1-\frac{\left(v_{p}-v_{\rho}\right) \gamma_{\rho} v_{\rho}}{\gamma c_{0}^{2}}\right]^{-1}
$$

For $I_{5}$, a simple shift of variables of integration $y^{\prime}=y+y^{\prime \prime}$ shows that $I_{5}$ is independent of $y$ :

$$
\begin{aligned}
I_{5}= & \int_{-\tilde{C} L_{x}}^{\tilde{C} L_{x}} d y^{\prime \prime} \frac{\sqrt{y^{\prime \prime 2}+\left(x-x^{\prime}\right)^{2}}}{2\left(x-x^{\prime}\right)^{2}+y^{\prime \prime 2}} \\
& \times\left[1-\frac{\gamma_{\rho} v_{\rho}}{\gamma c_{0}^{2}} \frac{\left(v_{p}-v_{\rho}\right) y^{\prime \prime 2}}{2\left(x-x^{\prime}\right)^{2}+y^{\prime \prime 2}}-\left(\frac{v_{\rho} \gamma_{\rho}}{c_{0} \gamma}\right)^{2} \frac{\left(x-x^{\prime}\right)^{2} y^{\prime \prime 2}}{\left[2\left(x-x^{\prime}\right)^{2}+y^{\prime \prime 2}\right]^{2}}\right]^{-1}
\end{aligned}
$$

Inserting these results $\mathrm{C} 8, \mathrm{C} 10$, and $\mathrm{C} 9$ into our

expression (C1), and using that result in our expression VI.3 for $\left\langle(\delta N)^{2}\right\rangle$, we obtain

$$
\begin{aligned}
& \left\langle(\delta N)^{2}\right\rangle \approx \frac{D_{p} \rho_{c}^{2} m^{2}}{2 \pi \gamma}\left\{\int_{0}^{L_{x}} d x \int_{0}^{L_{x}} d x^{\prime} \int_{0}^{L_{y}} d y\left(\ln \left(\frac{y}{L_{x}}\right)+\ln \left(\frac{L_{y}-y}{L_{x}}\right)-2 \ln \tilde{C}\right)\left[1-\frac{\left(v_{p}-v_{\rho}\right) \gamma_{\rho} v_{\rho}}{\gamma c_{0}^{2}}\right]^{-1}\right. \\
& \left.+\int_{0}^{L_{x}} d x \int_{0}^{L_{x}} d x^{\prime} \int_{0}^{L_{y}} d y \int_{-\tilde{C} L_{x}}^{\tilde{C} L_{x}} d y^{\prime \prime} \frac{\sqrt{y^{\prime \prime 2}+\left(x-x^{\prime}\right)^{2}}}{2\left(x-x^{\prime}\right)^{2}+y^{\prime \prime 2}} \times\left[1-\frac{\gamma_{\rho} v_{\rho}}{\gamma c_{0}^{2}} \frac{\left(v_{p}-v_{\rho}\right) y^{\prime \prime 2}}{\left[2\left(x-x^{\prime}\right)^{2}+y^{\prime \prime 2}\right]}-\left(\frac{v_{\rho} \gamma_{\rho}}{c_{0} \gamma}\right)^{2} \frac{\left(x-x^{\prime}\right)^{2} y^{\prime \prime 2}}{\left[2\left(x-x^{\prime}\right)^{2}+y^{\prime \prime 2}\right]^{2}}\right]^{-1}\right\}
\end{aligned}
$$

The integrals over $x$ and $x^{\prime}$ in the (triple) integral in the first line of this expression trivially give a factor of
$L_{x}^{2}$, since the integrand is independent of $x$ and $x^{\prime}$. The remaining integral over $y$ is elementary. The net result is 


$$
\begin{aligned}
& \int_{0}^{L_{x}} d x \int_{0}^{L_{x}} d x^{\prime} \int_{0}^{L_{y}} d y\left(\ln \left(\frac{y}{L_{x}}\right)+\ln \left(\frac{L_{y}-y}{L_{x}}\right)-2 \ln \tilde{C}\right)\left[1-\frac{\left(v_{p}-v_{\rho}\right) \gamma_{\rho} v_{\rho}}{\gamma c_{0}^{2}}\right]^{-1} \\
& =2 L_{x}^{2} L_{y}\left(\ln \left(\frac{L_{y}}{\tilde{C} L_{x}}\right)-1\right)\left[1-\frac{\left(v_{p}-v_{\rho}\right) \gamma_{\rho} v_{\rho}}{\gamma c_{0}^{2}}\right]^{-1} .
\end{aligned}
$$

The $y$ integral in the remaining (quadruple) integral which appears on the second line in equation (C11) can be done immediately, since the integrand is independent of $y$, yielding a factor of $L_{y}$. The remaining triple integral over $x, x^{\prime}$, and $y^{\prime \prime}$ can be done by changing variables of integration to new, rescaled variables $u_{x}, u_{y}$, and $u_{y}^{\prime}$ via

$$
x \equiv L_{x} u_{x} \quad, \quad x^{\prime} \equiv L_{x} u_{x}^{\prime} \quad, \quad y^{\prime \prime} \equiv L_{x} u_{y} .
$$

This gives

$$
\begin{aligned}
\int_{0}^{L_{x}} d x \int_{0}^{L_{x}} d x^{\prime} \int_{0}^{L_{y}} d y \int_{-\tilde{C} L_{x}}^{\tilde{C} L_{x}} d y^{\prime \prime} \frac{\sqrt{y^{\prime \prime 2}+\left(x-x^{\prime}\right)^{2}}}{2\left(x-x^{\prime}\right)^{2}+y^{\prime \prime 2}} \times & {\left[1-\frac{\gamma_{\rho} v_{\rho}}{\gamma c_{0}^{2}} \frac{\left(v_{p}-v_{\rho}\right) y^{\prime \prime 2}}{\left[2\left(x-x^{\prime}\right)^{2}+y^{\prime \prime 2}\right]}-\left(\frac{v_{\rho} \gamma_{\rho}}{c_{0} \gamma}\right)^{2} \frac{\left(x-x^{\prime}\right)^{2} y^{\prime \prime 2}}{\left[2\left(x-x^{\prime}\right)^{2}+y^{\prime \prime 2}\right]^{2}}\right]^{-1} } \\
= & \tilde{C}^{\prime} L_{x}^{2} L_{y}
\end{aligned}
$$

where

$$
\begin{aligned}
\tilde{C}^{\prime} \equiv & \int_{0}^{1} d u_{x} \int_{0}^{1} d u_{x}^{\prime} \int_{-C}^{C} d u_{y} \frac{\sqrt{\left(u_{x}-u_{x}^{\prime}\right)^{2}+u_{y}^{2}}}{u_{y}^{2}+2\left(u_{x}-u_{x}^{\prime}\right)^{2}} \\
& {\left[1-\frac{\gamma_{\rho} v_{\rho}}{\gamma c_{0}^{2}} \frac{\left(v_{p}-v_{\rho}\right) u_{y}^{2}}{2\left(u_{x}-u_{x}^{\prime}\right)^{2}+u_{y}^{2}}-\left(\frac{v_{\rho} \gamma_{\rho}}{c_{0} \gamma}\right)^{2} \frac{u_{y}^{2}\left(u_{x}-u_{x}^{\prime}\right)^{2}}{\left[2\left(u_{x}-u_{x}^{\prime}\right)^{2}+u_{y}^{2}\right]^{2}}\right]^{-1} }
\end{aligned}
$$

is an $\mathcal{O}(1)$ constant.

Comparing $(\mathrm{C} 12)$ and $(\mathrm{C} 14)$, we see that the first line of (C11) actually dominates the second in the small as- pect ratio limit $L_{x} \ll L_{y}$ that we are considering here. Therefore, we obtain, in the limit of small aspect ratio $\left(\alpha_{A} \ll 1\right)$ :

$$
\sqrt{\left\langle(\delta N)^{2}\right\rangle} \approx \frac{\rho_{c} m \sqrt{\tilde{C}^{\prime \prime} D_{p}}}{\sqrt{2 \pi \gamma}} \sqrt{L_{x}^{2} L_{y} \ln \left(\frac{L_{y}}{L_{x}}\right)} \approx \frac{\rho_{c} m \sqrt{\tilde{C}^{\prime \prime} D_{p}}}{\sqrt{2 \pi \gamma}} L_{y}^{3 / 2} \alpha_{A} \sqrt{\ln \left(\frac{1}{\alpha_{A}}\right)},
$$

where we have defined

$$
\tilde{C}^{\prime \prime} \equiv 2\left[1-\frac{\left(v_{p}-v_{\rho}\right) \gamma_{\rho} v_{\rho}}{\gamma c_{0}^{2}}\right]^{-1}
$$

Note that $C^{\prime}$ is an $O(1)$, parameter-dependent but aspect ratio and box size independent constant.

This can be rewritten in terms of the mean particle number $\bar{N}$ in the same area Ausing VI.19, which gives

$$
\Delta N=\sqrt{\left\langle(\delta N)^{2}\right\rangle}=\left(\frac{\rho_{c} m}{\rho_{0}^{3 / 4}}\right)\left(\sqrt{\frac{C^{\prime} D_{p}}{2 \pi \gamma}}\right) \bar{N}^{3 / 4} \alpha_{A}^{1 / 4} \sqrt{\ln \left(\frac{1}{\alpha_{A}}\right)} .
$$


Appendix F: Bulk velocity correlations in the $y$ - and $z$-directions

In this section we calculate the bulk velocity correlations in the $y$ and $z$-directions. We first work out the velocity correlations in the $y$-direction.

Using (VII.14) and VII.15), we see that the velocity correlation in the $y$ direction is given by

$$
\begin{aligned}
C_{y y}^{v}\left(v_{0}\left(t-t^{\prime}\right) \hat{\mathbf{x}}, z, z, t-t^{\prime}\right)= & C_{y y}^{p p}\left(v_{0}\left(t-t^{\prime}\right) \hat{\mathbf{x}}, z, z, t-t^{\prime}\right)+C_{y y}^{\rho \rho}\left(v_{0}\left(t-t^{\prime}\right) \hat{\mathbf{x}}, z, z, t-t^{\prime}\right) \\
= & v_{0}^{2} \int \frac{d \omega}{2 \pi} \frac{d^{2} q}{(2 \pi)^{2}} \exp \left[i\left(\omega-v_{0} q_{x}\right)\left(t-t^{\prime}\right)-2 q z\right] C_{p p}(\mathbf{q}, \omega)\left(1-\frac{2 q_{y}^{2} z}{q}+\frac{q_{y}^{4} z^{2}}{q^{2}}\right) \\
& +v_{a}^{\prime 2} \int \frac{d \omega}{2 \pi} \frac{d^{2} q}{(2 \pi)^{2}} \exp \left[i\left(\omega-v_{0} q_{x}\right)\left(t-t^{\prime}\right)-2 q z\right] C_{\rho \rho}(\mathbf{q}, \omega) \frac{z^{2} q_{x}^{2} q_{y}^{2}}{q^{2}} .
\end{aligned}
$$

Making the change of variables VII.18 as before, we

find that $C_{y y}^{v}\left(v_{0}\left(t-t^{\prime}\right) \hat{\mathbf{x}}, z, z, t-t^{\prime}\right)$ also obeys a scaling law:

$$
C_{y y}^{v}\left(v_{0}\left(t-t^{\prime}\right) \hat{\mathbf{x}}, z, z, t-t^{\prime}\right)=\left(\frac{D_{p} v_{0}}{z}\right) F_{y y}^{p p}\left(\frac{v_{0}\left|t-t^{\prime}\right|}{z}\right)+\left(\frac{D_{p} \rho_{c}^{2} v_{a}^{\prime 2}}{v_{0} z}\right) F_{y y}^{\rho \rho}\left(\frac{v_{0}\left|t-t^{\prime}\right|}{z}\right)
$$

where now the dimensionless scaling functions are given by

$$
\begin{aligned}
& F_{y y}^{p p}\left(u_{r}\right)=\int \frac{d \Omega}{2 \pi} \frac{d^{2} Q}{(2 \pi)^{2}} \exp \left[i\left(\Omega-Q_{x}\right) u_{r}-2 Q\right] H_{p p}\left(\frac{\Omega}{Q}, \theta_{\mathbf{Q}} ;\left\{\frac{v_{\rho}}{v_{0}}, \frac{v_{p}}{v_{0}}, \frac{\gamma}{v_{0}}, \frac{c_{0}}{v_{0}}\right\}\right)\left(1-2 \frac{Q_{y}^{2}}{Q}+\frac{Q_{y}^{4}}{Q^{2}}\right)\left(\frac{1}{Q^{2}}\right) . \\
& F_{y y}^{\rho \rho}\left(u_{r}\right)=\int \frac{d \Omega}{2 \pi} \frac{d^{2} Q}{(2 \pi)^{2}} \exp \left[i\left(\Omega-Q_{x}\right) u_{r}-2 Q\right] H_{\rho \rho}\left(\frac{\Omega}{Q}, \theta_{\mathbf{Q}} ;\left\{\frac{v_{\rho}}{v_{0}}, \frac{v_{p}}{v_{0}}, \frac{\gamma}{v_{0}}, \frac{c_{0}}{v_{0}}\right\}\right) \frac{Q_{x}^{2} Q_{y}^{2}}{Q^{4}} .
\end{aligned}
$$

The limiting behaviors of these scaling functions can be obtained by an almost identical analysis to that used for $F_{x x}^{p p}\left(u_{r}\right)$, with the result:

$$
F_{y y}^{p p}\left(u_{r}\right)= \begin{cases}A_{y y}^{p p}, & u_{r} \ll 1 \\ \frac{\left(B_{y y}^{p p}\right)_{1}}{u_{r}}+\frac{\left(B_{y y}^{p p}\right)_{2}}{u_{r}^{2}}+\frac{\left(B_{y y}^{p p}\right)_{3}}{u_{r}^{3}}, & u \gg 1\end{cases}
$$

and

$$
F_{y y}^{\rho \rho}\left(u_{r}\right)=\left\{\begin{array}{lll}
A_{y y}^{\rho \rho} & , & u_{r} \ll 1 \\
\frac{B_{y y}^{\rho \rho}}{u_{r}^{3}} & , & u_{r} \gg 1
\end{array}\right.
$$

where the constants $A_{y y}^{p p}, A_{y y}^{\rho \rho},\left(B_{y y}^{p p}\right)_{1},\left(B_{y y}^{p p}\right)_{2},\left(B_{y y}^{p p}\right)_{3}$, and $B_{y y}^{\rho \rho}$ are given by

$$
\begin{aligned}
& A_{y y}^{p p}=\int \frac{d \Omega}{2 \pi} \frac{d^{2} Q}{(2 \pi)^{2}} e^{-2 Q} H_{p p}\left(\frac{\Omega}{Q}, \theta_{\mathbf{Q}} ;\left\{\frac{v_{\rho}}{v_{0}}, \frac{v_{p}}{v_{0}}, \frac{\gamma}{v_{0}}, \frac{c_{0}}{v_{0}}\right\}\right)\left(1-2 \frac{Q_{y}^{2}}{Q}+\frac{Q_{y}^{4}}{Q^{2}}\right)\left(\frac{1}{Q^{2}}\right), \\
& A_{y y}^{\rho \rho}=\int \frac{d \Omega}{2 \pi} \frac{d^{2} Q}{(2 \pi)^{2}} e^{-2 Q} H_{\rho \rho}\left(\frac{\Omega}{Q}, \theta_{\mathbf{Q}} ;\left\{\frac{v_{\rho}}{v_{0}}, \frac{v_{p}}{v_{0}}, \frac{\gamma}{v_{0}}, \frac{c_{0}}{v_{0}}\right\}\right) \frac{Q_{x}^{2} Q_{y}^{2}}{Q^{4}} .
\end{aligned}
$$




$$
\begin{aligned}
\left(B_{y y}^{p p}\right)_{1} & =\int \frac{d \Omega^{\prime}}{2 \pi} \frac{d^{2} Q^{\prime}}{(2 \pi)^{2}} \exp \left[i\left(\Omega^{\prime}-Q_{x}^{\prime}\right)\right] H_{p p}\left(\frac{\Omega^{\prime}}{Q^{\prime}}, \theta_{\mathbf{Q}^{\prime}} ;\left\{\frac{v_{\rho}}{v_{0}}, \frac{v_{p}}{v_{0}}, \frac{\gamma}{v_{0}}, \frac{c_{0}}{v_{0}}\right\}\right) \frac{1}{Q^{\prime 2}}, \\
\left(B_{y y}^{p p}\right)_{2} & =-2 \int \frac{d \Omega^{\prime}}{2 \pi} \frac{d^{2} Q^{\prime}}{(2 \pi)^{2}} \exp \left[i\left(\Omega^{\prime}-Q_{x}^{\prime}\right)\right] H_{p p}\left(\frac{\Omega^{\prime}}{Q^{\prime}}, \theta_{\mathbf{Q}^{\prime}} ;\left\{\frac{v_{\rho}}{v_{0}}, \frac{v_{p}}{v_{0}}, \frac{\gamma}{v_{0}}, \frac{c_{0}}{v_{0}}\right\}\right) \frac{Q_{y}^{\prime 2}}{Q^{\prime 3}}, \\
\left(B_{y y}^{p p}\right)_{3} & =\int \frac{d \Omega^{\prime}}{2 \pi} \frac{d^{2} Q^{\prime}}{(2 \pi)^{2}} \exp \left[i\left(\Omega^{\prime}-Q_{x}^{\prime}\right)\right] H_{p p}\left(\frac{\Omega^{\prime}}{Q^{\prime}}, \theta_{\mathbf{Q}^{\prime}} ;\left\{\frac{v_{\rho}}{v_{0}}, \frac{v_{p}}{v_{0}}, \frac{\gamma}{v_{0}}, \frac{c_{0}}{v_{0}}\right\}\right) \frac{Q_{y}^{\prime 4}}{Q^{\prime 4}} \\
B_{y y}^{\rho \rho} & =\int \frac{d \Omega^{\prime}}{2 \pi} \frac{d^{2} Q^{\prime}}{(2 \pi)^{2}} \exp \left[i\left(\Omega^{\prime}-Q_{x}^{\prime}\right)\right] H_{\rho \rho}\left(\frac{\Omega^{\prime}}{Q^{\prime}}, \theta_{\mathbf{Q}^{\prime}} ;\left\{\frac{v_{\rho}}{v_{0}}, \frac{v_{p}}{v_{0}}, \frac{\gamma}{v_{0}}, \frac{c_{0}}{v_{0}}\right\}\right) \frac{Q_{x}^{\prime 2} Q_{y}^{\prime 2}}{Q^{\prime 4}} .
\end{aligned}
$$

As we found earlier, all of these constants are again functions of all of the ratios $\frac{v_{\rho}}{v_{0}}, \frac{v_{p}}{v_{0}}, \frac{\gamma}{v_{0}}$, and $\frac{c_{0}}{v_{0}}$, and will again be of $O(1)$ when all of these ratios are of $\mathcal{O}(1)$.

However, the important point is that the dominant term in $\left(\mathrm{C} 12\right.$ at large $u$, which is obviously the $\left(B_{y y}^{p p}\right)_{1}$ term, is clearly not integrable over $u$ all the way out to $u=\infty$; its integral diverges logarithmically. We will show in the next subsection that this leads to superdiffusive motion in the $y$ direction. Thus, taking this together with our result for velocity fluctuations in the $x$ direction, we see that there will be superdiffusion for both directions parallel to the surface.

Interestingly, the superdiffusion in the $x$-direction is not correlated with that in the $y$-direction. To see this, we calculate the cross-correlation function $C_{x y}^{v}$. Using VII.14 and VII.15), we see that this is given by

$$
\begin{aligned}
C_{x y}^{v}\left(v_{0}\left(t-t^{\prime}\right) \hat{\mathbf{x}}, z, z, t-t^{\prime}\right) & =C_{y y}^{p \rho}\left(v_{0}\left(t-t^{\prime}\right) \hat{\mathbf{x}}, z, z, t-t^{\prime}\right) \\
& =v_{0} v_{a}^{\prime} \int \frac{d \omega}{2 \pi} \frac{d^{2} q}{(2 \pi)^{2}} \exp \left[i\left(\omega-v_{0} q_{x}\right)\left(t-t^{\prime}\right)-2 q z\right] C_{p \rho}(\mathbf{q}, \omega)\left(1-q z+\frac{2 q_{x}^{2} q_{y}^{2} z^{2}}{q^{2}}\right) .
\end{aligned}
$$

This vanishes due to the fact that $C_{p \rho}(\mathbf{q}, \omega)$ is odd in $q_{y}$; see (V.7) above. Thus, there is no cross-correlation between the superdiffusion in the $x$-direction and that in the $y$-direction.
We now turn to the motion of the tracer particles in the $z$-direction. The relevant velocity correlation using VII.14, can be expressed as

$$
C_{z z}^{v}\left(v_{0}\left(t-t^{\prime}\right) \hat{\mathbf{x}}, z, z, t-t^{\prime}\right)=C_{z z}^{p p}\left(v_{0}\left(t-t^{\prime}\right) \hat{\mathbf{x}}, z, z, t-t^{\prime}\right)+C_{z z}^{\rho \rho}\left(v_{0}\left(t-t^{\prime}\right) \hat{\mathbf{x}}, z, z, t-t^{\prime}\right)
$$

We evaluate each of the two terms in C15. From

VII.15, we see that $C_{z z}^{v}\left(v_{0}\left(t-t^{\prime}\right) \hat{\mathbf{x}}, z, z, t-t^{\prime}\right)$ is given by

$$
\begin{aligned}
& C_{z z}^{v}\left(v_{0}\left(t-t^{\prime}\right) \hat{\mathbf{x}}, z, z, t-t^{\prime}\right) \\
& =v_{0}^{2} \int \frac{d \omega}{2 \pi} \frac{d^{2} q}{(2 \pi)^{2}} \exp \left[i\left(\omega-v_{0} q_{x}\right)\left(t-t^{\prime}\right)-2 q z\right] C_{p p}(\mathbf{q}, \omega) q_{y}^{2} z^{2}+v_{a}^{\prime 2} \int \frac{d \omega}{2 \pi} \frac{d^{2} q}{(2 \pi)^{2}} \exp \left[i\left(\omega-v_{0} q_{x}\right)\left(t-t^{\prime}\right)-2 q z\right] C_{\rho \rho}(\mathbf{q}, \omega) q_{x}^{2} z^{2}
\end{aligned}
$$

Making the same change of variables VII.18 that we made when we were analyzing $C_{x x}^{v}\left(v_{0}\left(t-t^{\prime}\right) \hat{\mathbf{x}}, z, z, t-t^{\prime}\right)$, we find that $C_{z z}^{v}\left(v_{0}\left(t-t^{\prime}\right) \hat{\mathbf{x}}, z, z, t-t^{\prime}\right)$ obeys an almost identical scaling law: 


$$
C_{z z}^{v}\left(v_{0}\left(t-t^{\prime}\right) \hat{\mathbf{x}}, z, z, t-t^{\prime}\right)=\left(\frac{D_{p} v_{0}}{z}\right) F_{z z}^{p p}\left(\frac{v_{0}\left|t-t^{\prime}\right|}{z}\right)+\left(\frac{D_{p} \rho_{c}^{2} v_{a}^{\prime 2}}{v_{0} z}\right) F_{z z}^{\rho \rho}\left(\frac{v_{0}\left|t-t^{\prime}\right|}{z}\right)
$$

where now the dimensionless scaling functions are given by

$$
\begin{aligned}
& F_{z z}^{p p}\left(u_{r}\right)=\int \frac{d \Omega}{2 \pi} \frac{d^{2} Q}{(2 \pi)^{2}} \exp \left[i\left(\Omega-Q_{x}\right) u_{r}-2 Q\right] H_{p p}\left(\frac{\Omega}{Q}, \theta_{\mathbf{Q}} ;\left\{\frac{v_{\rho}}{v_{0}}, \frac{v_{p}}{v_{0}}, \frac{\gamma}{v_{0}}, \frac{c_{0}}{v_{0}}\right\}\right) \frac{Q_{y}^{2}}{Q^{2}}, \\
& F_{z z}^{\rho \rho}\left(u_{r}\right)=\int \frac{d \Omega}{2 \pi} \frac{d^{2} Q}{(2 \pi)^{2}} \exp \left[i\left(\Omega-Q_{x}\right) u_{r}-2 Q\right] H_{\rho \rho}\left(\frac{\Omega}{Q}, \theta_{\mathbf{Q}} ;\left\{\frac{v_{\rho}}{v_{0}}, \frac{v_{p}}{v_{0}}, \frac{\gamma}{v_{0}}, \frac{c_{0}}{v_{0}}\right\}\right) \frac{Q_{x}^{2}}{Q^{2}}
\end{aligned}
$$

The limiting behaviors of these scaling functions can be obtained by an almost identical analysis to that used for $F_{x x}^{p p}(u)$, with almost identical results:

$$
F_{z z}^{p p}\left(u_{r}\right)=\left\{\begin{array}{lll}
A_{z z}^{p p} & , & u_{r} \ll 1 \\
\frac{B_{z z}^{p p}}{u_{r}^{3}} & , & u_{r} \gg 1
\end{array}\right.
$$

and

$$
F_{z z}^{\rho \rho}\left(u_{r}\right)= \begin{cases}A_{z z}^{\rho \rho}, & u_{r} \ll 1 \\ \frac{B_{z z}^{\rho \rho}}{u_{r}^{3}} & , \quad u_{r} \gg 1\end{cases}
$$

with

$$
\begin{aligned}
& A_{z z}^{p p}=\int \frac{d \Omega}{2 \pi} \frac{d^{2} Q}{(2 \pi)^{2}} e^{-2 Q} H_{p p}\left(\frac{\Omega}{Q}, \theta_{\mathbf{Q}} ;\left\{\frac{v_{\rho}}{v_{0}}, \frac{v_{p}}{v_{0}}, \frac{\gamma}{v_{0}}, \frac{c_{0}}{v_{0}}\right\}\right) \frac{Q_{y}^{2}}{Q^{2}}, \\
& A_{z z}^{\rho \rho}=\int \frac{d \Omega}{2 \pi} \frac{d^{2} Q}{(2 \pi)^{2}} e^{-2 Q} H_{\rho \rho}\left(\frac{\Omega}{Q}, \theta_{\mathbf{Q}} ;\left\{\frac{v_{\rho}}{v_{0}}, \frac{v_{p}}{v_{0}}, \frac{\gamma}{v_{0}}, \frac{c_{0}}{v_{0}}\right\}\right) \frac{Q_{x}^{2}}{Q^{2}},
\end{aligned}
$$

and

$$
\begin{aligned}
& B_{z z}^{p p}=\int \frac{d \Omega^{\prime}}{2 \pi} \frac{d^{2} Q^{\prime}}{(2 \pi)^{2}} \exp \left[i\left(\Omega^{\prime}-Q_{x}^{\prime}\right)\right] H_{p p}\left(\frac{\Omega^{\prime}}{Q^{\prime}}, \theta_{\mathbf{Q}^{\prime}} ;\left\{\frac{v_{\rho}}{v_{0}}, \frac{v_{p}}{v_{0}}, \frac{\gamma}{v_{0}}, \frac{c_{0}}{v_{0}}\right\}\right) \frac{Q_{y}^{\prime 2}}{Q^{\prime 2}}, \\
& B_{z z}^{\rho \rho}=\int \frac{d \Omega^{\prime}}{2 \pi} \frac{d^{2} Q^{\prime}}{(2 \pi)^{2}} \exp \left[i\left(\Omega^{\prime}-Q_{x}^{\prime}\right)\right] H_{\rho \rho}\left(\frac{\Omega^{\prime}}{Q^{\prime}}, \theta_{\mathbf{Q}^{\prime}} ;\left\{\frac{v_{\rho}}{v_{0}}, \frac{v_{p}}{v_{0}}, \frac{\gamma}{v_{0}}, \frac{c_{0}}{v_{0}}\right\}\right) \frac{Q_{x}^{\prime 2}}{Q^{\prime 2}},
\end{aligned}
$$

Note that, as we found for the $x x$ correlations, $A_{z z}^{p p}$, $A_{z z}^{\rho \rho}, B_{z z}^{p p}$ and $B_{z z}^{\rho \rho}$ are functions of all of the ratios $\frac{v_{\rho}}{v_{0}}$, $\frac{v_{p}}{v_{0}}, \frac{\gamma}{v_{0}}$, and $\frac{c_{0}}{v_{0}}$, and will be of $O(1)$ when all of these ratios are of $O(1)$.

Note also that the scaling function for the $z$ direction falls off sufficiently rapidly as a function of its argument $u$ that their integral over all $u$ converges. We show in Section (VII) that this implies that the motion of tracer particles in the $z$ direction is simply conventional diffusion. Furthermore, we also showed there that the diffusion constant for the $z$-direction is independent of the height $z$ of the particles above the surface.
[1] K. Kruse, J.F. Joanny, F. Jülicher, J. Prost, and K. Sekimoto, Asters, vortices, and rotating spirals in active gels of polar filaments, Phys. Rev. Lett. 92, 078101(2004). 
[2] K. Kruse, J.F. Joanny, F. Jülicher, J. Prost, and K. Sekimoto, Generic theory of active polar gels: a paradigm for cytoskeletal dynamics, Eur. Phys. J. E, 16, 5, (2005).

[3] H. Wioland, F. G. Woodhouse, J. Dunkel, J. O. Kessler, and R.E. Goldstein, Confinement stabilizes a bacterial suspension into a spiral vortex, Phys. Rev. Lett., 110, 268102, (2013).

[4] D. Saintillan, and M. J. Shelley, Instabilities, pattern formation, and mixing in active suspensions, Phys. Fluids 20, 123304, (2008).

[5] Y. Hatwalne, S. Ramaswamy, M. Rao, S. Madan and A. Simha, Rheology of active-particle suspensions, Phys. Rev. Lett. 92, 118101 (2004).

[6] S. Saha, R. Golestanian, and S. Ramaswamy, Clusters, asters, and collective oscillations in chemotactic colloids, Phys. Rev. E, 89, 062316, (2014).

[7] B. Liebchen, D. Marenduzzo, I. Pagonabarraga, and M.E. Cates, Clustering and Pattern Formation in Chemorepulsive Active Colloids, Phys. Rev. Lett. 115, 258301(2015).

[8] V. Narayan, S. Ramaswamy, and N. Menon, Long-Lived Giant Number Fluctuations in a Swarming Granular Nematic, Science 317, 105, (2007).

[9] L.J. Daniels, Y. Park, T. C. Lubensky, and D. J. Durian, Dynamics of gas-fluidized granular rods, Phys. Rev. E79, 041301(2009).

[10] A. Baskaran, and M.C. Marchetti, Hydrodynamics of self-propelled hard rods, Phys. Rev. E 77, 011920 (2008).

[11] P.G. de Gennes and J. Prost, The Physics of Liquid Crystals (Oxford University Press, Oxford, 1995)

[12] T. Vicsek, A. Czirók, E. Ben-Jacob, I. Cohen, and O. Shochet, Novel type of phase transition in a system of self-driven particles, Phys. Rev. Lett. 75, 1226, (1995).

[13] J. Toner and Y. Tu, Long-Range Order in a TwoDimensional Dynamical XY Model: How Birds Fly Together, Phys. Rev. Lett. 75, 4326 (1995).

[14] J. Toner and Y. Tu, Flocks, herds, and schools: A quantitative theory of flocking, Phys. Rev. E 58, (1998).

[15] J. Toner, Y. Tu, and S. Ramaswamy, Hydrodynamics and phases of flocks, Annals of Physics 318, 170 (2005).

[16] R.A. Simha and S. Ramaswamy, Hydrodynamic fluctuations and instabilities in ordered suspensions of selfpropelled particles, Phys. Rev. Lett. 89, 058101(2002).

[17] H. Chaté, F. Ginelli, G. Gregoire and F. Raynaud, Collective motion of self-propelled particles interacting without cohesion, Phys Rev E 77, 046113 (2008); F. Ginelli, The Physics of the Vicsek model, Eur. Phys. J. Special Topics 225, 2099 (2016).

[18] J. Toner, Giant number fluctuations in dry active polar fluids: A shocking analogy with lightning rods, J. Chem. Phys. 150, 154120 (2019).

[19] S. Ramaswamy, R.A. Simha, and J. Toner, Active nematics on a substrate: Giant number fluctuations and long-time tails, Europhys. Lett. 62, 196 (2003).

[20] C. Pérez-González, R. Alert, C. Blanch-Mercader, M. Gómez-González, T. Kolodziej, E. Bazellieres, J. Casademunt, and X. Trepat, Active wetting of epithelial tissues, Nature Physics 15, 1 (2019).

[21] R. Alert, and X. Trepat, Physical Models of Collective Cell Migration, Annual Review of Condensed Matter Physics 11, 1 (2020).

[22] C. Wolgemuth, E. Hoiczyk, D. Kaiser, and G. Oster, How myxobacteria glide, Current Biology, 12, 369 (2002).

[23] E. Lushi, and H. Wioland, and R. Goldstein, Fluid flows created by swimming bacteria drive self-organization in confined suspensions, Proceedings of the National Academy of Sciences 111, 9733 (2014).

[24] H. H. Wensink, J. Dunkel, S. Heidenreich, K. Drescher, R. E. Goldstein, H. Löwen, and J. M Yeomans, Mesoscale turbulence in living fluids, Proceedings of the National Academy of Sciences 109, 14308 (2012).

[25] D. B. Stein, G. D. Canio, E. Lauga, M. J. Shelley, and R. E. Goldstein, Swirling Instability of the Microtubule Cytoskeleton, Phys. Rev. Lett. 126, 028103 (2021).

[26] A. Doostmohammadi, J. Ignés-Mullol, J. M. Yeomans, and F. Sagués, Active nematics, Nature Communications 9, 3246 (2018).

[27] V. Schaller, and A.R. Bausch, Topological defects and density fluctuations in collectively moving systems, Proceedings of the National Academy of Sciences 110, 4488 (2013).

[28] A. Bricard, J.-B. Caussin, N. Desreumaux, O. Dauchot, and D. Bartolo, Emergence of macroscopic directed motion in populations of motile colloids, Nature 503, 95 (2013).

[29] D. Geyer, A. Morin and D. Bartolo, Sounds and hydrodynamics of polar active fluids, Nature Materials 17, 789 (2018).

[30] M. R. Nejad, A. Doostmohammadi and J. M. Yeomans, Memory effects, arches and polar defect ordering at the cross-over from wet to dry active nematics, Soft Matter 17, 2500 (2021).

[31] A. Doostmohammadi, M. F. Adamer, S. P. Thampi, and J. M. Yeomans, Stabilization of active matter by flowvortex lattices and defect ordering, Nature Communications 7, 1 (2016)

[32] N. D. Mermin and H. Wagner, Absence of Ferromagnetism or Antiferromagnetism in One- or TwoDimensional Isotropic Heisenberg Models, Phys. Rev. Lett. 17, 1133 (1966); P. C. Hohenberg, Existence of Long-Range Order in One and Two Dimensions, Phys. Rev. 158, 383 (1967); N. D. Mermin, Absence of Ordering in Certain Classical Systems, J. Math. Phys. 8, 1061 (1967).

[33] Exceptions to this result are two dimensional crystals (see, e.g., 34]) and fluctuating tethered membranes (see, e.g., [35]).

[34] B. I. Halperin and D. R. Nelson, Theory of TwoDimensional Melting, Phys. Rev. Lett. 41, 121 (1978).

[35] Y. Kantor, M. Kardar, and D. R. Nelson, Statistical Mechanics of Tethered Surfaces, Phys. Rev. Lett. 57, 791 (1986).

[36] The only example of a finite $Q$ as $q \rightarrow 0$ we know of is smectic liquid crystals; see [37.

[37] G. F. Mazenko, S. Ramaswamy and J. Toner, Viscosities diverge as $1 / \omega$ in smectic-A liquid crystals, Phys. Rev. Lett. 49, 51 (1982); G. F. Mazenko, S. Ramaswamy and J. Toner, Breakdown of conventional hydrodynamics for smectic-A, hexatic-B, and cholesteric liquid crystals, Phys. Rev. A28, 1618 (1983).

[38] It is a peculiarity of our problem, and in particular of the fact that damping in this system scales as $q$, rather than the usual $q^{2}$, that a damping coefficient can have the dimensions of a speed, rather than a diffusion constant.

[39] These are not actually the sound speeds one would obtain from the real part of the eigenfrequencies $\omega$.

[40] J. Toner, Harvard University Ph. D. Thesis, unpublished (1981). 
[41] P. M. Chaikin and T. C. Lubensky, Principles of condensed matter physics (Cambridge University Press, Cambridge UK, 1995).

[42] Y. Zhu and S. Granick, No-Slip Boundary Condition Switches to Partial Slip When Fluid Contains Surfactant, Langmuir 18, 10058 (2002).

[43] In most of the literature, the $\zeta$ terms in this expression are taken to be the divergence of a tensor: that is, instead of those terms, one writes $\partial_{j}\left(\zeta p_{i} p_{j}\right)=\zeta(\rho)\left(\hat{\mathbf{p}} \cdot \nabla_{s}\right) p_{i}+$ $\zeta(\rho) p_{i}\left(\nabla_{s} \cdot \hat{\mathbf{p}}\right)+p_{i}\left(\hat{\mathbf{p}} \cdot \nabla_{s}\right) \zeta(\rho)$, which is a special case (specifically, $\zeta_{1}=\zeta_{2}=\zeta$ ) of the more general expression we have used here. That our more general expression is actually allowed - i.e., that these terms need not add up to the divergence of a tensor in non-equilibrium systemswas first recognized by 44 .

[44] A. Maitra, P. Srivastava, M.C. Marchetti, J.S. Lintuvuori, S. Ramaswamy, and M. Lenz, A nonequilibrium force can stabilize 2D active nematics, Proceedings of the National Academy of Sciences 115, 6934 (2018).

[45] P.C. Martin, O. Parodi, and P.S. Pershan, Unified hydrodynamic theory for crystals, liquid crystals, and normal fluids, Phys. Rev. A 6, 2401 (1972).

[46] E. Bertin, M. Droz, G. Gregoire, Hydrodynamic equations for self-propelled particles: microscopic derivation and stability analysis, J. Phys. A: Math. Theor. 42, 445001 (2009).

[47] S. Mishra, A. Baskaran, and M. C. Marchetti, Fluctuations and pattern formation in self-propelled particles, Phys. Rev. E 81, 061916 (2010).

[48] S. Shankar, S. Ramaswamy, and M. C. Marchetti, Lownoise phase of a two-dimensional active nematic system, Phys. Rev. E 97, 012707 (2018); S. Mishra, A. Baskaran, and M. C. Marchetti, Fluctuations and pattern formation in self-propelled particles, Phys. Rev. E 81, 061916 (2010).

[49] The only equilibrium systems we know of that shows giant number fluctuations away from a critical point are superfluids. See, e.g., Ref. 41] above.

[50] The apparent divergence of these kernels as $\mathbf{r}_{\|} \rightarrow \mathbf{0}$ will actually be cutoff at small $\mathbf{r}_{\|}$if we impose an ultraviolet cutoff on our wavevector integrals, as we should, since our hydrodynamic theory does not apply out to arbitrarily large wave numbers (or, equivalently, down to arbitrarily small wavelengths. Hence, the forms (IX.16) and IX.4 should be considered as large distance expansions of the kernel, asymptotically valid for $r_{\perp} \gg a$, where $a$ is a short-distance cutoff. An explicit demonstration that these kernels do not divegre as $\mathbf{r} \rightarrow 0$ if we impose an ultraviolet cutoff is given for an explicit choice of cutoff in appendix $\mathrm{D}$

[51] D. Forster, D. R. Nelson, and M. J. Stephen, Largedistance and long-time properties of a randomly stirred fluid, Phys. Rev. A 16, 732 (1977).

[52] A. Maitra, P. Srivastava, M. C. Marchetti, S. Ramaswamy, and M. Lenz, Swimmer Suspensions on Substrates: Anomalous Stability and Long-Range Order, Phys. Rev. Lett. 124, 028002 (2020).

[53] G. I. Taylor, Dispersion of soluble matter in solvent flowing slowly through a tube, Proc. Roy. Soc. A., 219, 186203 (1953).

[54] P. Guillamata, J. Ignés-Mullola, and F. Saguésa, Control of active liquid crystals with a magnetic field, Proceedings of the National Academy of Sciences 113, 5498 (2016). 